\title{
Conformational Dynamics of Living Polymers
}

\author{
Dissertation \\ zur Erlangung des \\ mathematisch-naturwissenschaftlichen Doktorgrades \\ "Doctor rerum naturalium" \\ der Georg-August-Universität Göttingen \\ im Promotionsprogramm ProPhys \\ der Georg-August University School of Science (GAUSS)
}

vorgelegt von

Ali Malek

aus Teheran/Iran

Göttingen, 2018 


\section{Betreuungsausschuss}

Prof. Dr. Reiner Kree,

Institut für Theoretische Physik,

Georg-August-Universität Göttingen

Prof. Dr. Marcus Müller,

Institut für Theoretische Physik,

Georg-August-Universität Göttingen

\section{Mitglieder der Prüfungskommission}

Prof. Dr. Reiner Kree,

Institut für Theoretische Physik,

Georg-August-Universität Göttingen

Prof. Dr. Marcus Müller,

Institut für Theoretische Physik,

Georg-August-Universität Göttingen

Weitere Mitglieder der Prüfungskommission

Prof. Dr. Annette Zippelius,

Institut für Theoretische Physik,

Georg-August-Universität Göttingen

Prof. Dr. Sarah Köster,

Institute for X-Ray Physics,

Georg-August-Universität Göttingen

PD. Dr. Salvatore R. Manmana,

Institut für Theoretische Physik,

Georg-August-Universität Göttingen

Dr. Claus Heussinger,

Institut für Theoretische Physik,

Georg-August-Universität Göttingen 
Tag der mündlichen Prüfung: 
To Mina, Negar and my beloved parents 


\section{Acknowledgements}

First of all I would like to express my sincere thanks to Prof. Dr. Reiner Kree, not only for his excellent supervising and full scientific support on my Ph.D project but also for his patience and his father like spiritual supports. I will always remember that and be grateful for it.

I extent my gratitude to Prof. Dr. Marcus Müller, the co-supervisor of the thesis.

I also have to thank the members of my PhD committee, Prof. Dr. Annette Zippelius, Prof. Dr. Sarah Köster, Dr. Claus Heussinger and Dr. Salvatore R. Manmana for their assistance.

I express my appreciation to my mother and my father for their love and "never ending" care throughout my life.

Finally, I dedicate this work to my wife Mina and my daughter Negar who shared patiently every moment and waited hardly till it comes to an end. This journey would not have been possible without your supports. Thank you. 


\section{Contents}

1 Introduction $\quad 1$

2 Model and Roadmap $\quad 6$

2.1 Rouse model . . . . . . . . . . . . . . . . . . . 6

2.1.1 Discrete chain . . . . . . . . . . . . . . . . 6

2.1 .2 Continuous chain . . . . . . . . . . . . . . . . . 11

2.1.3 Non-dimensionalization . . . . . . . . . . . . . . . . 12

2.2 Solution of the Rouse model . . . . . . . . . . . . . . . . . . . . . . 14

2.2 .1 Discrete chain . . . . . . . . . . . . . . . . . 14

2.2 .2 Continuous chain . . . . . . . . . . . . . . . 15

2.3 Observables . . . . . . . . . . . . . . . . . . . . 17

2.3.1 Center of mass and end-to-end vector . . . . . . . . . . . 17

2.3.2 Segmental motion: short- and long- time Behavior . . . . . . . . . 18

2.3 .3 Tension dynamics . . . . . . . . . . . . . . . . 20

2.4 Polymerization reactions . . . . . . . . . . . . . . . . 23

2.4.1 Markov model of chemical reactions . . . . . . . . . . . . . 23

2.4 .2 Chain growth reactions . . . . . . . . . . . . . . . . 24

2.5 Model of conformational dynamics of a living Rouse chain . . . . . . . 28

2.5.1 Couplings between chemical and conformational dynamics . . . . . 28

2.5.2 Living Rouse chain: discrete model . . . . . . . . . . . . . . . 29

2.5.3 Living Rouse chain: continuous model . . . . . . . . . . . . . . 30

2.5.4 Non-stationary growth . . . . . . . . . . . . . . . . . . . . . . . . 33

2.6 Roadmap . . . . . . . . . . . . . . . . . . . . . . . 34

3 Methods $\quad \mathbf{3 6}$

3.1 Simulation algorithm . . . . . . . . . . . . . . . 36

3.1 .1 Description of Algorithm I . . . . . . . . . . . . . . 37

3.1.2 Problem with Euler schemes . . . . . . . . . . . . . . . . . 39

3.1.3 More efficient algorithm for non-stationary growth . . . . . . . . 39

3.2 Collection of analytical tools . . . . . . . . . . . . . 41

3.2.1 Green's function for moving boundary problems . . . . . . . . . 42

3.2 .2 Landau transformation . . . . . . . . . . . . . . . . . . . . 42

3.2.3 Perturbation methods . . . . . . . . . . . . . . . . . 44

4 Dynamics of a Free Living Rouse Chain $\quad 46$

4.1 Regimes of non-stationary growth . . . . . . . . . . . . . . . . . . . 46

4.2 Slow polymerization . . . . . . . . . . . . . . . 48 
4.3 Balanced polymerization: exact solution for $\alpha=1 / 2 \ldots \ldots$. . . . . 50

4.4 Fast polymerization and exact solution for $\alpha=1 \ldots \ldots . \ldots . . \ldots 52$

4.5 Dynamical transitions . . . . . . . . . . . . . . . . . . 55

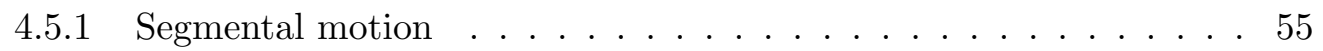

4.5 .2 Center of mass . . . . . . . . . . . . . . 58

5 Dragged Living Rouse Chain $\quad 60$

5.1 Fixed length chain . . . . . . . . . . . . . . . . . . 6 61

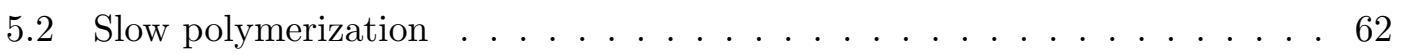

5.3 Balanced polymerization . . . . . . . . . . . . . . . . 64

5.4 Fast polymerization . . . . . . . . . . . . . . . 65

5.5 Summary of the analytical results . . . . . . . . . . . . . . . . . . . . . . . . . . 67

5.6 Dynamical transitions . . . . . . . . . . . . . . . . . . . 6 67 67

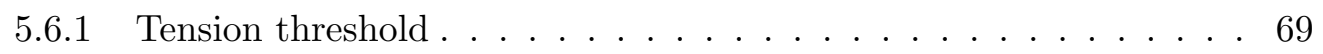

5.6 .2 End-to-end distance . . . . . . . . . . . . . . . . . . 70

5.6.3 Base and tip in the center of mass reference frame . . . . . . . 71

5.6 .4 The shape of the chain . . . . . . . . . . . . . . . 71

5.6.5 Coupling between center of mass and tip . . . . . . . . . . . 72

5.6 .6 Base point $R(0, t) \ldots \ldots \ldots \ldots \ldots 74$

6 Living Rouse Chain in Shear Flow $\quad \mathbf{7 6}$

6.1 Some elements of rheology . . . . . . . . . . . . . . . . 76

6.1 .1 Steady shear flow . . . . . . . . . . . . . . . 76

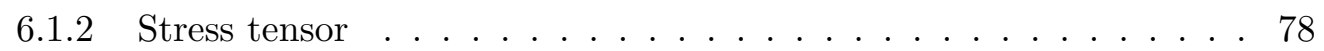

6.1 .3 Viscometric functions . . . . . . . . . . . . . . . . . 79

6.1.4 Rheology of fixed length polymers . . . . . . . . . . . 80

6.2 Slow polymerization regime . . . . . . . . . . . . . . . 83

6.3 Simulation algorithm . . . . . . . . . . . . . . . . 88

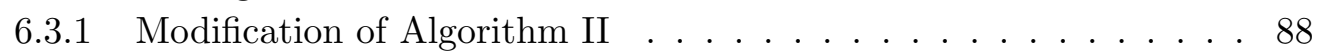

6.4 Coil - stretch transition . . . . . . . . . . . . . . . . . 91

$\begin{array}{lll}7 & \text { Summary and Conclusion } & 96\end{array}$

A First Correction for the Regular Perturbation Method 100

$\begin{array}{lr}\text { B A useful transformation } & 102\end{array}$

$\begin{array}{lr}\text { Bibliography } & 106\end{array}$ 


\section{Chapter 1}

\section{Introduction}

Polymerization is outstanding among chemical reaction processes due to its importance for technical applications as well as for an understanding of complex processes in living matters. A sequence of polymerization reactions forms large molecules from reactive molecular sub-units called monomers. If the sub-units are all of the same type, we call them homopolymers. Alternatively, the polymer chain may be a combination of two or more structural units known as copolymers.

Dependent on the polymerization process and the ingredients of the reaction, polymers may possess different architectures. The simplest architecture is called linear, in which all monomers are arranged along a single backbone. However, there might also exist more complicated structures such as star polymers, ring polymers, polymer block comb polymers, etc. These architectures are mostly formed, as a result of branching points on the polymer. Complicated structures may lead to different physical properties compared to linear polymers.

Polymerization is classified into chain-growth and step-growth polymerization[1]. In chain-growth polymerization, the production of polymers from free monomers takes place in three stages[2]: First the polymerization has to be initiated by producing an active site, from which the polymer chain grows. The active site is highly reactive and is able to bind to a single monomer. The bound monomer makes the new end of the chain and becomes active and this step is repeated and monomers are added one by one to one end (or both ends) of the polymer. The successive addition of monomers to the active end of the polymer is called propagation stage. Finally, the polymerization may be terminated due to various reasons, for instance, by reacting the active sites of two chains. 
In contrast to chain growth polymerization, in a step-growth polymerization, reaction occurs between any of the different-sized species present in the reaction system. One proceeds from monomer to dimer, trimer, tetramer, pentamer, and etc., and all the oligomers react with each other, until eventually large-sized polymer molecules are formed.

In this thesis, we will study the dynamics of linear homopolymers, which undergo chaingrowth polymerization. Such situations are of importance, because they constitute a large part of the technically and biologically relevant processes.

In 2001 alone, 36 percent of polymers in the United States were produced by free radical polymerization, one of the most widely used chain-growth polymerization techniques. polyethylene and polystyrene, the most widespread polymers in our daily life, both are produced using free radical polymerization.

As an example, consider the free radical polymerization of polyethylene from sub-units called ethylene or ethene $\mathrm{CH}_{2}=\mathrm{CH}_{2}$. Here the initiation stage is a two-step process. First $\mathrm{O}-\mathrm{O}$ single bonds of the peroxide molecules are broken leading to production of free radicals $\mathrm{RO}^{\bullet}$ inside the system. These free radicals are very unstable and highly reactive. When an $\mathrm{RO}^{\bullet}$ gets close to an ethylene monomer, it attacks to its double bond, breaks it and attaches to the monomer. The resulting molecule is the active center of the polymer:

$$
\mathrm{RO}^{\bullet}+\mathrm{CH}_{2}=\mathrm{CH}_{2} \stackrel{k_{i}}{\longrightarrow} \mathrm{RO}-\mathrm{CH}_{2}-\mathrm{CH}_{2} \bullet
$$

and is capable of further growth.

In the propagation stage, monomers are bound to the active center. The active center breaks the double bond of a monomer, and consequently the monomer attaches to it. Again one electron remains unpaired and is prone to further polymerization. This process continues and, while it is occurring, the chain length grows:

$$
\mathrm{RO}-\left[\mathrm{CH}_{2}-\mathrm{CH}_{2}\right]_{n} \bullet+\mathrm{CH}_{2}=\mathrm{CH}_{2} \stackrel{\mathrm{k}_{p}}{\longrightarrow} \mathrm{RO}-\left[\mathrm{CH}_{2}-\mathrm{CH}_{2}\right]_{n+1} \bullet
$$

In this thesis, we will call each of these addition processes a polymerization step or a polymerization event. Note that the rate of each polymerization step is taken to be equal and independent of the polymer length[1]. In free radical polymerization, the most frequent termination occurs when two free radicals bind together. This reaction is energetically favorable, because now the unpaired electrons in two free radicals become paired and drop to lower energy level.

Another important example of chain-growth polymerization is actin polymerization in cellular environments. In living cells, polymerization of actin filaments (F-actins) from globular actins (G-actins) is essential in various cellular activities such as cell motility, 
cell division and protrusions of neural cells[3]. Actin polymerization, like any other chain growth polymerization, is characterized by three stages: initiation, propagation and termination. The G-actin building blocks, however, are themselves (hetero)-polymers, and therefore, F-actin is a supra-molecular polymer. In polymerization of actin filaments, after formation of the so-called actin nuclei, which is, practically, the active center of the polymer, filaments propagate rapidly by addition of G-actins at one (or maybe both ends) of the chain called a barbed end. Inside cell, polymerization of actin is usually a reversible process. In other words, monomers also may dissociate from the tip. However, the rate of polymerization at the barbed end is about 50-500 times faster than depolymerization[4-6]. Therefore, it makes sense if we neglect the depolymerization process in such systems. Finally, the polymerization may be blocked by binding capping proteins to the barbed end[7, 8].

Another important example, is the supra-molecular polymerization of microtubules from sub-units called tubulin dimers. Micorotubules are one of the stiffest structural elements found in animal cells. They polymerize by addition of free tubulin to the tip of the filament - a process which is very similar to a chain-growth polymerization. The polymerization keeps on going and, consequently, the concentration of free dimers decreases until this concentration reaches a critical value. Below the critical concentration, the regular polymerization is interrupted and the depolymerization phase starts.

There are important situations, where the termination processes are blocked (at least during the time of observation) by some mechanism, and the propagation carries on until all monomers in the reaction chamber are used up. This type of polymerization is called "living". The term was introduced by M. Szwarc [9]. It has become an important tool in technical control of polymerization processes, for example to produce polymer systems with narrow molecular weight distributions[10] or systems with complex polymeric architectures[11]. To synchronize the propagation stages of a many-chain system requires de-initiation process to be fast. In this thesis we concentrate on growth and conformation of a single chain, and consequently use the term living polymerization in a slightly broader sense, irrespective of the time-scale of initiation. For us, the important feature of living polymerization is, that the polymer chain growth continues for ever, if the system is constantly fed with monomers. Our focus will be on the conformational dynamical properties of polymerizing chains during the propagation stage. We always assume that the chain is already initiated and, on the timescale of our observation, termination processes are absent.

Understanding the evolution of such reacting polymers necessitates the knowledge of the conformational evolution of non-reacting polymers as well as the underlying mechanism of chemical reactions. These two topics are both well established and have 
been extensively studied in physics and chemistry. The well-known standard models of conformational dynamics such as the Rouse model[12], Zimm model[13] and reptation model $[14,15]$ are set up for non-reacting chains of fixed molecular structure. These models all describe the dynamics of fixed-length polymers as Brownian particles which are connected together. Theoretical reaction models, on the other hand, are usually formulated as stochastic processes in the space of chemical sequences of monomers [16]. The majority of studies, however, have considered the two processes separately.

In two extreme cases, studying the dynamics of (de-)polymerizing polymers seems to be straightforward: Either the conformational dynamics of the chain evolves over a much longer timescale than the chemical reactions, or the opposite situation occurs, in which the chemical reactions take place much more slowly than the conformational transition of the polymer molecule. In both of these cases, the timescales involved in the chemical reaction and that involved in the relaxation process of the polymer chain trough equilibrium are sharply separated. Therefore, in the former case, one assumes that between two chemical reactions the polymer relaxes spontaneously to its equilibrium state. The latter can be treated by assuming that the chain conformation does not change considerably between two chemical reactions and therefore, the conformation is considered to be rigid.

However, there are situations, where the conformational evolution of the chain and the chemical reaction occur at comparable rates and the decoupled approach gives no insight into the real process. In a typical polymerization process of polystyrene with a monomer concentration of $3 \mathrm{M}$ and the characteristic polymerization rate of $4.4 * 10^{-1} \mathrm{~m}^{3} / \mathrm{mol} \cdot \mathrm{s}$ [17], the rate of polymerization for each of the initiated chains is approximated to be 1000 monomer per second[18]. On the other hand, the typical relaxation time of polystyrene varies in a range between 1 to 10 seconds, depending on the experimental conditions such as temperature and solvent viscosity[19].

For actins, the situation is more or less the same. For example, actin filaments in a bath of G-actins with a concentration of $4 \mu \mathrm{M}[20]$ polymerize at a rate of about $50-500$ monomers per second[4], while the typical relaxation time of such a filament has been measured to be about 15 seconds[21].

In cellular environment, monomers or regulators of reactions are provided in limited amount and degrade or diffuse away from the reaction volume and may lead to decreasing reaction rates[20, 22-25]. In all these situations, the two dynamics of the polymer, namely the conformational evolution and the chemical reaction dynamics, may be coupled in several non-trivial ways. For instance, the motion of the polymer ends (where active centers are positioned) as reactants, may affect the rate of the polymerization 
reaction. On the other hand, the evolution of the polymer length due to the polymerization reaction will definitely change the conformational dynamics of polymer chains. For such a mutual coupling between these two dynamics, we expect complex dynamical behavior of a polymerizing chain.

To study the coupling between the conformation of the polymer and the chemical reaction, we have extended the Rouse model to cases where the polymer undergoes polymerization. In the next two chapters, we introduce the model of conformational dynamics of a growing flexible chain, step by step. To keep the discussion self-contained and to fix the notation, we start with a brief review of the Rouse model of conformational dynamics. This topic is discussed in detail in many textbooks on polymer dynamics (see references $[15,26]$ ), but as we will make use of exact solutions of this model in our simulation method, we will discuss several variants, including different boundary conditions. Next, based on the stochastic description of chemical reactions, we present a model for the chain-length evolution due to the chemical reactions. Finally, on the basis of these two models, we extend the Rouse model, to describe the dynamics of a polymerizing polymer. This model will be presented both in discrete and continuous versions.

The rest of this thesis is devoted to the study the dynamical properties of a Rouse chain, which is growing deterministically in time. We will consider various experimentally relevant scenarios and study the impact of the non-stationary growth on the conformational dynamics of living Rouse chains. A more detailed roadmap about the thesis is available in Section 2.6. 


\section{Chapter 2}

\section{Model and Roadmap}

We model the conformational dynamics of a living polymer from two well-studied ingredients: the Rouse dynamics of a flexible chain of fixed length and the Markovian description of polymerization reaction. We review both of these parts before we combine them into our theoretical model, which is the basis of the subsequent work.

\section{$2.1 \quad$ Rouse model}

\subsubsection{Discrete chain}

The Rouse model is a very simple dynamic generalization of simplest model of equilibrium conformations of a single flexible polymer chain in solution. This simplest model neglects all non-bonding interactions between monomers (like, for example, excluded volume interactions due to steric repulsion). The energetically degenerate conformations form random walks in position space. If the polymer is described by a set of $M+1$ molecular monomer positions, and these molecular monomers are grouped into a sequence of a $N+1$ beads, also called quasi-monomers, which consists of a sufficiently large number of molecular monomers in sequence, the central limit theorem assures that the bond vector $\vec{b}$, connecting two successive beads, is distributed according to the Gaussian statistics:

$$
p(\vec{b})=\left(\frac{3}{2 \pi b^{2}}\right)^{\frac{3}{2}} \exp \left[-\frac{3|\vec{b}|^{2}}{2 b^{2}}\right] .
$$

Note that $p(\vec{b})$ is characterized by a single parameter $b>0$, which is also called the Kuhn length and is chosen so, that the average $\left\langle\vec{b}^{2}\right\rangle=b^{2}$. We can interpret the joint distribution $p\left(\vec{b}_{1}, \cdots, \vec{b}_{N}\right)$ as the Boltzmann equilibrium distribution of bead conformations, if we 
identify $\vec{b}_{n}=\vec{R}_{n}-\vec{R}_{n-1}$ :

$$
p\left(\vec{R}_{0}, \cdots, \vec{R}_{N}\right)=\left(\frac{3}{2 \pi b^{2}}\right)^{3 N / 2} e^{-\beta \mathcal{H}_{0}}
$$

with:

$$
\mathcal{H}_{0}=\frac{1}{2} \sum_{n=1}^{N} \frac{3\left|\vec{b}_{n}\right|^{2}}{\beta b^{2}}
$$

The statistical properties of such a system is equivalent to a chain of beads connected via entropic Hookian springs with the spring constant (see Fig. 2.1):

$$
k:=\frac{3 k_{B} T}{b^{2}} .
$$

This model is called Gaussian chain model. We can also introduce the tension along

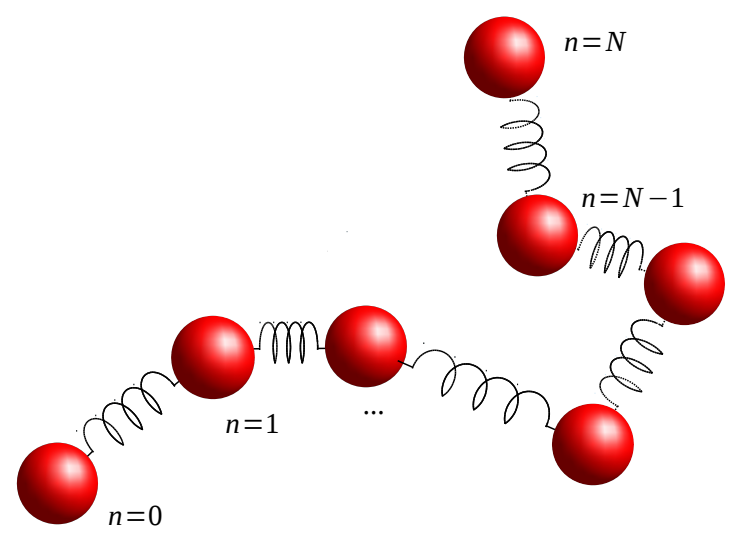

FIGURE 2.1: In the Rouse model, the polymer is modelled as a set of beads connected via springs, all floating in a concentrated fluid, where the inertia of each bead can be neglected compared to the friction encountered by each bead.

the chain as $\vec{t}_{n}=-k \vec{b}_{n}$. As we will see in Chapter 5 , understanding the distribution of the tension along the polymer gives an important insight into the structural properties of the chain.

A simple polymer dynamics based upon this Gaussian model has been introduced by P. E. Rouse [12]. In the Rouse model we assume that the beads behave like (over-damped) Brownian particles, coupled by entropic springs between adjacent beads. The dynamics of chain conformations, thus, becomes a system of linear Langevin equations[27]. 
We can collect all $3 N+3$ Cartesian components of the bead positions into a single column vector,

$$
\overrightarrow{\boldsymbol{R}}:=\left[\begin{array}{c}
\vec{R}_{0} \\
\vdots \\
\vec{R}_{N}
\end{array}\right] .
$$

This may be read as an $N$ component vector (indexed by Latin characters) of 3component position vectors of monomers. The Cartesian components will be represented by Greek indices $\alpha, \beta$.

Later, the number of $N+1$ beads may change. Then we will make it explicit as an index and write $\overrightarrow{\boldsymbol{R}}_{N}$. The Langevin equations may be written as

$$
\zeta \frac{\mathrm{d} \overrightarrow{\boldsymbol{R}}}{\mathrm{d} t}=\overrightarrow{\boldsymbol{F}}_{i n}(\overrightarrow{\boldsymbol{R}})+\overrightarrow{\boldsymbol{F}}_{e x}(\overrightarrow{\boldsymbol{R}})+\overrightarrow{\boldsymbol{f}}
$$

where the friction force $\zeta \frac{\mathrm{d} \overrightarrow{\boldsymbol{R}}}{\mathrm{d} t}$ on the left hand side is balanced by all the internal forces $\overrightarrow{\boldsymbol{F}}_{\text {in }}(\overrightarrow{\boldsymbol{R}}):=\nabla_{\boldsymbol{R}} \mathcal{H}_{0}$ exerted from other subunits of the chain, by externally applied forces $\overrightarrow{\boldsymbol{F}}_{\text {ex }}(\overrightarrow{\boldsymbol{R}})$ and by Langevin stochastic forces $\overrightarrow{\boldsymbol{f}}$ from the solvent. $\overrightarrow{\boldsymbol{F}}_{i n}$, as obtained from the Gaussian chain model, is linear in $\overrightarrow{\boldsymbol{R}}$ and may be written as:

$$
\overrightarrow{\boldsymbol{F}}_{\text {in }}=-\frac{3 k T}{b^{2}} \hat{\boldsymbol{A}} \overrightarrow{\boldsymbol{R}}
$$

The coupling between the beads can be represented as an $(N+1) \times(N+1)$ matrix $\hat{\boldsymbol{A}}$, which in textbooks is called the Rouse matrix. As we will see later, different experimental set-ups refer to different Rouse matrices. The stochastic force $\vec{f}$ is a delta-correlated Gaussian random force field and can be fully characterized by its first and second moments:

$$
\begin{aligned}
\left\langle f_{n, \alpha}\right\rangle & =0 \\
\left\langle f_{n, \alpha}(t) f_{n^{\prime}, \beta}\left(t^{\prime}\right)\right\rangle & =2 \zeta k_{B} T \delta_{\alpha, \beta} \delta_{n, n^{\prime}} \delta\left(t-t^{\prime}\right)
\end{aligned}
$$

The prefactor of the second moment, is fixed by Einstein's relation, which guarantees that the stationary distribution of the Langevin process is the Boltzmann distribution given in Eq.(2.2)[15]. In Eq.(2.6), the only non-linearity can arise from the external force $\overrightarrow{\boldsymbol{F}}_{\text {ex }}$ caused for example, by nonlinear flow fields in microfluidic devices[28, 29]. In this thesis, however, we are only concerned with linear flows or other external forces linear in $\vec{R}$.

The Rouse model neglects (i) non-linearities of the chemical bonding forces, (ii) entanglement effects, (iii) effects of inertia, and (iv) all non-bonding and all solvent-mediated interactions. The neglected effects may lead to different scaling behaviour of physical 
properties with chain length, and seem to restrict the applicability of the model to special situations (theta solvent) or to single chain properties in melts[15].

As the most simplistic model developed to describe the dynamics of polymers, the Rouse model may prove to have shortcomings. For example, for extremely long chains, where the entanglement effects are important or in a dilute solutions, where the excluded volume effects has to be taken into account[15, 30], the simple Rouse model does not seem to be applicable. Nevertheless, the Rouse model is still widely used by polymer chemists and experimental physicists to successfully describe the dynamics of polymer chains. For example R. Shusterman et al. analyized a single strand DNA using the Rouse model[31]; K. L. Sebastian modeled a double strand DNA as two Rouse chains interacting with each other[32], and T. Glomann et al. described the dynamics of a synthetic polymer Polyethylene Glycol using the Rouse model[33].

For our theoretical analysis, this model is of particular importance, because its linear structure allows us to construct efficient simulation algorithm and to find reliable approximate solution for the conformational dynamics of a living chain.

In studying the dynamics of polymers one can imagine various scenarios. For example we may study the dynamics of a chain in a fluid free of any external force. Or one might study the dynamics of a chain clamped at both ends. Or one may be interested in rheological properties of polymers and study the properties of a single chain under shear flow. All these situations may affect $\overrightarrow{\boldsymbol{F}}_{\text {in }}, \overrightarrow{\boldsymbol{F}}_{\text {ex }}$ or both of them. In the following we formulate some of the experimentally relevant set-ups.

- Free chain: The simplest case is a single chain in a solvent with $\boldsymbol{F}_{e x}=0$. For the interior beads, i.e. $n \neq 0, N$, the springs couple the dynamics of the $n^{\text {th }}$ bead to the $n-1^{\text {th }}$ and $n+1^{\text {th }}$ bead:

$$
\vec{F}_{n, i n}=-k\left(2 \vec{R}_{n}-\vec{R}_{n+1}-\vec{R}_{n-1}\right)
$$

At the boundaries we can write:

$$
\begin{array}{r}
F_{0, i n}=-k\left(\vec{R}_{0}-\vec{R}_{1}\right) \\
F_{N, \text { in }}=-k\left(\vec{R}_{N}-\vec{R}_{N-1}\right)
\end{array}
$$


Therefore the Rouse Matrix $\hat{\boldsymbol{A}}$ defined in Eq.(2.7) can be written as:

$$
\hat{\boldsymbol{A}}=\left[\begin{array}{cccccc}
1 & -1 & 0 & 0 & \cdots & 0 \\
-1 & 2 & -1 & 0 & \cdots & \vdots \\
0 & -1 & 2 & -1 & \cdots & 0 \\
0 & \cdots & -1 & 2 & -1 & 0 \\
\vdots & \cdots & 0 & -1 & 2 & -1 \\
0 & \cdots & 0 & 0 & -1 & 1
\end{array}\right]
$$

In Sec.2.2.1 we will discuss the exact solution for a chain of fixed length.

- Dragged polymer: Many efforts have been undertaken to understand the static and dynamical behavior of polymer chains dragged at one end[34, 35]. Studying polymers under an external fixed force provides understanding about the tension propagation along the polymer. For a chain dragged via a constant force $\vec{F}$ acting on the first bead, the external force vector is:

$$
\vec{F}_{e x, \alpha}=\delta_{\alpha, 0} \vec{F}
$$

- One end grafted from or onto a surface: For technical applications, understanding the physics of polymer brushes is of great importance. A brush is a set of chains with one end attached to a surface, while the other end is free. The first step in understanding the dynamics of brushes is to neglect the intermolecular interaction between different chains and therefore reduce the problem to a single chain problem. Here, for the sake of simplicity and without loss of generality, we assumed that the grafted end is fixed at the origin. For such a chain, one can write $\vec{R}_{i=0}(t)=\overrightarrow{0}$.

- Both ends grafted: One interesting example for a polymer chain which is grafted at both ends onto a surface is a grafted polymer ring. A grafted ring onto a surface can be formulated by putting the constraint

$$
\vec{R}_{0}(t)=\vec{R}_{N}(t)=\overrightarrow{0}
$$

- Polymer in flow: In studying of the rheological properties of a polymeric fluid, it is common to impose a flow field in the fluid and study the response of the system to this distortion. If we neglect the interactions between different chains in the polymer solution, we can restrict our focus to a single chain under such a flow. In general, considering an external velocity field $\vec{v}(\vec{r}, t)$, the external force $\overrightarrow{\boldsymbol{F}}_{\text {ex }}$ can be 
expressed as:

$$
\overrightarrow{\boldsymbol{F}}_{e x}=\zeta\left[\begin{array}{c}
\vec{v}\left(\vec{R}_{0}, t\right) \\
\vdots \\
\vec{v}\left(\vec{R}_{N}, t\right)
\end{array}\right] .
$$

For the $n^{t h}$ bead, one can write the force balance relation of Eq.(2.6) as:

$$
\zeta\left(\partial_{t} \vec{R}_{n}-\vec{v}\left(\vec{R}_{n}, t\right)\right)=\vec{F}_{n, i n}+\vec{f}_{n}
$$

Here, the left hand side shows, that the flow field changes the friction experienced by the bead due to the change in the relative velocity between the bead and the solvent. Depending on the nature of the flow field, it can be linear or nonlinear in the $\boldsymbol{R}$. In Chapter 6 we will study the dynamics of growing polymer chains under a special, but still very commonly used flow field, namely steady shear flow.

\subsubsection{Continuous chain}

On scales, which are much longer than the Kuhn length $b$, it is possible to describe the dynamics of the polymer in a continuum limit, for which the chain is considered as a curve $\vec{R}(s, t)$ in space. A simple minded first approach would be to let $N \rightarrow \infty$ and $b \rightarrow 0$, keeping $N b=l$ finite, but this would imply the mean-squared end-to-end distance of the chain $\left\langle R_{e e}^{2}\right\rangle=N b^{2}=0$. Instead, we can introduce a coordinate $s=n b^{2}$. Then, in the formal limit, the Gaussian Hamiltonian becomes $\beta H_{0}=\frac{3}{2 b^{2}} \sum_{n}\left(R_{n}-\right.$ $\left.R_{n-1}\right)^{2}=\frac{3}{2} \sum_{n} b^{2}\left(\frac{R_{n}-R_{n}-1}{b^{2}}\right)^{2} \rightarrow \frac{3}{2} \int_{0}^{l}\left(\partial R(s) / \partial_{s}\right)^{2} d s$. Note that in the equation above, without introducing new variables we replaced $\vec{R}_{n}$ by $\vec{R}(s, t)$, which is the continuum representation of the chain in space and time.

The evolution equation for the position of internal beads (excluding the boundary, i.e. $s=0$ and $s=l$ )

$$
\zeta \mathrm{d} \vec{R}_{n} / \mathrm{d} t=\left(3 k_{B} T / b^{2}\right)\left(\frac{\vec{R}_{n+1}+\vec{R}_{n-1}-2 \vec{R}_{n}}{b^{4}} b^{4}\right)+\vec{f}_{n}(t)
$$

Now we can formally take the limit $b \rightarrow 0$ and get:

$$
\zeta^{*} \frac{\mathrm{d} R}{\mathrm{~d} t}=k^{*} \frac{\partial^{2} R}{\partial s^{2}}+\overrightarrow{\tilde{f}}
$$

with $\zeta^{*}=\zeta / b^{2}, k^{*}=3 k T$ and a rescaled noise. 
where $\overrightarrow{\tilde{f}}(s, t)$ is a rescaled noise, i.e. $\overrightarrow{\tilde{f}}(s, t):=\frac{\vec{f}(s, t)}{b^{2}}$. The moments of this noise field reads:

$$
\begin{aligned}
& \langle\overrightarrow{\tilde{f}}(s, t)\rangle=0 \\
& \left\langle\tilde{f}_{\alpha}(s, t) \tilde{f}_{\beta}\left(s^{\prime}, t^{\prime}\right)\right\rangle=2 k T \zeta^{*} \delta_{\alpha, \beta} \delta\left(s-s^{\prime}\right) \delta\left(t-t^{\prime}\right) .
\end{aligned}
$$

Here we used the continuum limit of the Kronecker delta function namely $\frac{\delta_{s, s^{\prime}}}{b} \rightarrow \delta\left(s-s^{\prime}\right)$.

We want to stress that the continuum limit has been introduced here in a purely heuristic manner. In fact, solution $\vec{R}(s, t)$ of Eq.(2.19) lack the regularity, which was implicitly assumed in our discussion. $\vec{R}(s, t)$ is only Hölder continuous with index $1 / 2$ in $s$ and $1 / 4$ in time[36]. Our arguments, however, do only need a mollified version of $\vec{R}$ and are thus untouched by the lack of regularity. Mollifying also corresponds to interpreting the continuum model as a coarse grained version of the discrete model, i.e. it reproduces the properties of the discrete model for conformations $\vec{R}(s, t)$, which vary very slowly between adjacent monomers.

Appropriate boundary conditions at $\vec{R}(n=0)$, grafted onto or from a point $\vec{r}_{0}$, are obviously given by $\vec{R}(n=0, t)=\vec{r}_{0}$ and are called Dirichlet boundary conditions. At a free end the balance of forces has to be considered. All the acting forces, i.e. the harmonic bonding forces, the friction forces and the stochastic forces produce finite line tensions, i.e. finite force per chemical length, but no additional forces located at the boundary. Thus if we integrate the equation (2.19), over a small s-interval including the boundary and let the length of the interval go to zero, we find that the tension $\vec{t}=k \partial_{n} \vec{R}$ has to vanish at the boundary: $\left.\partial_{n} \vec{R}\right|_{n=0}=0$. These boundary conditions are called von Neumann boundary conditions. Finally, if the end is dragged by a force $\vec{F}$ located at the boundary, the force balance requires: $\left.\partial_{n} \vec{R}\right|_{n=0}=\vec{F}$.

\subsubsection{Non-dimensionalization}

As a primary step in analyzing the polymer dynamics we choose appropriate scales for quantities with dimensions energy, length and time. It is natural to choose $k_{B} T$ and the Kuhn length $b$ as the basic energy and length units respectively. We also choose the basic time unit as the time it takes for a bead to diffuse a distance $b$ :

$$
\tau_{b}:=\frac{b^{2}}{3 D}
$$

with the diffusion constant $D=k_{B} T / \zeta$ which is equal to $\zeta / k$, if the spring are entropic springs with spring constant given in Eq.(2.4). In the following we non-dimensionalize 
all lengths, times and energies involved in the evolution equations (discrete and continuum) by the basic units introduced above. Introducing the asterisk letters for nondimensionalized variables, we get: $\overrightarrow{\boldsymbol{R}}^{*}:=\frac{\overrightarrow{\boldsymbol{R}}}{b}$ and $\overrightarrow{\boldsymbol{f}}^{*}:=\frac{b \vec{f}}{k_{B} T}$ which result in $\frac{\mathrm{d}}{\mathrm{d} t^{*}} \overrightarrow{\boldsymbol{R}}^{*}=$ $-\hat{\boldsymbol{A}} \overrightarrow{\boldsymbol{R}}^{*}+\overrightarrow{\boldsymbol{f}}^{*}$. The first and second moments of the noise $\overrightarrow{\boldsymbol{f}}_{N}^{*}$ become $\left\langle\overrightarrow{\boldsymbol{f}}_{N}^{*}\right\rangle=0$ and $\left\langle f_{n, \alpha}^{*}\left(t^{*}\right) f_{m, \beta}^{*}\left(t^{* \prime}\right)\right\rangle=2 \delta_{\alpha, \beta} \delta_{n, m} \delta\left(t^{*}-t^{* \prime}\right)$ respectively. For the continuum limit, let us re-introduced $n$ as a continuous non-dimensionalized parametrization $n:=\frac{s}{b^{2}}$. Putting everything together, the continuum version of the Rouse chain can ultimately be described as:

$$
\partial_{t^{*}} \vec{R}^{*}\left(n, t^{*}\right)=\partial_{n}^{2} \vec{R}^{*}\left(n, t^{*}\right)+\vec{f}^{*}\left(n, t^{*}\right)
$$

along with the noise correlation:

$$
\begin{aligned}
& \left\langle\vec{f}^{*}\left(n, t^{*}\right)\right\rangle=0 \\
& \left\langle f_{\alpha}^{*}\left(n, t^{*}\right) f_{\beta}\left(m, t^{* \prime}\right)\right\rangle=2 \delta_{\alpha, \beta} \delta(m-n) \delta\left(t^{*}-t^{* \prime}\right)
\end{aligned}
$$

and boundary conditions, like:

$$
\begin{aligned}
\left.\partial_{n} \vec{R}^{*}\right|_{n=0} & =0 \\
\left.\partial_{n} \vec{R}^{*}\right|_{n=N} & =0
\end{aligned}
$$

for a force free chain. During this work we will use both discrete and continuous descriptions interchangeably. To avoid confusion and keep notations simple, we drop the asterisks and only use the non-dimensionalized variables introduced in this section.

All in all, our non-dimensional equation of motion in discrete form reads:

$$
\partial_{t} \overrightarrow{\boldsymbol{R}}(t)=\hat{\boldsymbol{A}} \overrightarrow{\boldsymbol{R}}(t)+\overrightarrow{\boldsymbol{f}}(t)
$$

and in the continuum version:

$$
\partial_{t} \vec{R}(n, t)=\partial_{n}^{2} \vec{R}(n, t)+\vec{f}(n, t) .
$$

Due to the simple form of Eq.(2.29), we can map all the conformational properties of chains of different length $N$ to the properties of a chain of length 1 . So instead of $n$ we use $\sigma:=\frac{n}{N},(0 \leq \sigma \leq 1)$ which gives us $\partial_{\sigma}^{2} / N^{2}$ for the Laplace operator. Multiplying by $N^{2}$ and introducing $\tau:=\frac{t}{N^{2}}$ yields:

$$
\partial_{\tau} \vec{R}=\partial_{\sigma}^{2} \vec{R}+\sqrt{N} \vec{f}(\sigma, \tau)
$$


The stochastic term $\vec{f}(\sigma, \tau)$ is just the standard white noise with:

$$
\left\langle f_{\alpha}(\tau, \sigma) f_{\beta}\left(\tau^{\prime}, \sigma^{\prime}\right)\right\rangle=2 \delta_{\alpha, \beta} \delta\left(\tau-\tau^{\prime}\right) \delta\left(\sigma-\sigma^{\prime}\right) .
$$

Finally we can transform the position vector $\vec{R}$ as $\vec{\rho}:=\frac{\vec{R}}{\sqrt{N}}$ in order to obtain:

$$
\partial_{\tau} \vec{\rho}=\partial_{\sigma}^{2} \vec{\rho}+\vec{f}
$$

\subsection{Solution of the Rouse model}

\subsubsection{Discrete chain}

To solve the set of linear stochastic differential equations (2.28) one has to find the $N+1$ eigenstates $\phi_{0}, \cdots, \phi_{N}$ of the Rouse matrix:

$$
\hat{\boldsymbol{A}} \phi_{p}=c_{p} \phi_{p}
$$

If we expand the dynamical variables on this basis, we will have a decoupled set of ordinary differential equation for modes, which can be solved using various techniques. Finally, the position vectors can be obtained by transforming these modes back to the position space. For a force free Rouse chain, the $n^{\text {th }}$ component of $\phi_{\boldsymbol{p}}$ reads:

$$
\begin{aligned}
\phi_{n, 0} & :=\frac{1}{\sqrt{N+1}} \\
\phi_{n, p \neq 0} & :=\sqrt{\frac{2}{N+1}} \cos \left(\frac{p(2 n+1) \pi}{2(N+1)}\right),
\end{aligned}
$$

and the corresponding eigenvalues are:

$$
c_{p}=4 \sin ^{2}\left(\frac{p \pi}{2(N+1)}\right) \quad p=0,1, \cdots, N
$$

The components of $\overrightarrow{\boldsymbol{R}}$ can be written as:

$$
\begin{gathered}
\vec{R}_{n}(t)=\frac{1}{\sqrt{N+1}} \vec{X}_{0}(t)+\sqrt{\frac{2}{N+1}} \sum_{p=1}^{N} \vec{X}_{p}(t) \cos \frac{p(2 n+1) \pi}{2(N+1)} \\
\vec{f}_{n}(t)=\frac{1}{\sqrt{N+1}} \vec{\eta}_{0}(t)+\sqrt{\frac{2}{N+1}} \sum_{p=1}^{N} \vec{\eta}_{p}(t) \cos \frac{p(2 n+1) \pi}{2(N+1)}
\end{gathered}
$$

where $\vec{X}_{p}$ s are the modes of the position vector $\vec{R}_{n}$. The orthonormality of the basis leads to:

$$
\partial_{t} \vec{X}_{p}(t)=c_{p} \vec{X}_{p}(t)+\vec{\eta}_{p}(t) \quad p=0,1, \cdots, N
$$


with:

$$
\begin{aligned}
\left\langle\vec{\eta}_{p}(t)\right\rangle & =\overrightarrow{0} \\
\left\langle\eta_{\alpha, p}(t) \eta_{\beta, q}\left(t^{\prime}\right)\right\rangle & =2 \delta_{\alpha, \beta} \delta_{p, q} \delta\left(t-t^{\prime}\right)
\end{aligned}
$$

As one can see from Eq.(2.39), the set of $N+1$ coupled equations for $\vec{R}_{n}$ s is converted to a set of $N+1$ independent ordinary differential equations for the modes $\vec{X}_{p}(t)$, which can be solved using standard methods. The solutions read:

$$
\begin{aligned}
& \vec{X}_{0}(t)=\int_{t_{0}}^{t} \vec{\eta}_{p}\left(t^{\prime}\right) \mathrm{d} t^{\prime}+\vec{X}_{0}\left(t_{0}\right) \\
& \vec{X}_{p}(t)=\vec{X}_{p}\left(t_{0}\right) e^{-c_{p}\left(t-t_{0}\right)}+\int_{t_{0}}^{t} e^{-c_{p}\left(t-t^{\prime}\right)} \vec{\eta}_{p}\left(t^{\prime}\right) \mathrm{d} t^{\prime} \quad p \neq 0
\end{aligned}
$$

Multiplying both side of Eq.(2.43) by the fixed initial $\vec{X}_{p}\left(t_{0}\right)$ and averaging over $\vec{\eta}_{p}$ in Eq.(2.41), we can easily read off the autocorrelation function for each mode:

$$
\left\langle\vec{X}_{p}(t) \cdot \vec{X}_{p}\left(t_{0}\right)\right\rangle=\vec{X}_{p}^{2}\left(t_{0}\right) e^{-c_{p}\left(t-t_{0}\right)}
$$

The $p^{\text {th }}$ mode relaxes exponentially with the characteristic time scale:

$$
\tau_{p}=\frac{1}{c_{p}}=\left[4 \sin ^{2}\left(\frac{p \pi}{2(N+1)}\right)\right]^{-1} .
$$

For modes with $p \ll N$, this relaxation time can be approximated as:

$$
\tau_{p} \approx \frac{1}{p^{2}}\left(\frac{N}{\pi}\right)^{2}
$$

The longest relaxation time is called the Rouse time:

$$
\tau_{R}:=\frac{N^{2}}{\pi^{2}}
$$

which defines the relaxation timescale of the polymer chain as a whole.

\subsubsection{Continuous chain}

To solve the continuous model of the Rouse chain, the Rouse matrix $\hat{\boldsymbol{A}}$ is replaced by the Laplace operator $\partial^{2} / \partial n^{2}$ with appropriate boundary conditions. The eigenfunctions of this operator gives a complete orthonormal system. For a free chain, these eigenfunctions are $\phi_{p}(n) \propto \cos (p \pi n / N)$ with $p=0, \cdots, \infty$, whereas for a ring polymer grafted at $\vec{r}=\overrightarrow{0}$, the eigenfunctions are $\propto \sin (p \pi n / N)$ with $p=1, \cdots, \infty$. 
So for a free chain we can write:

$$
\vec{R}(n, t)=\vec{X}_{0}(t)+2 \sum_{p=1}^{\infty} \vec{X}_{p}(t) \cos \left(\frac{p \pi n}{N}\right) .
$$

The inhomogeneity $\vec{f}(n, t)$ can also be expanded in the same way:

$$
\vec{f}(n, t)=\vec{\eta}_{0}(t)+2 \sum_{p=1}^{\infty} \vec{\eta}_{p}(t) \cos \left(\frac{p \pi n}{N}\right)
$$

Substituting this equation into the evolution equation, and making use of the orthonormality of $\phi_{p} \mathrm{~s}$, the partial differential equation is converted to an infinite set of ordinary differential equations for the modes:

$$
\begin{aligned}
\partial_{t} \vec{X}_{0}(t) & =\vec{\eta}_{0}(t) \\
\partial_{t} \vec{X}_{p}(t) & =-k_{p}^{2} \vec{X}_{p}+\vec{\eta}_{p}(t)
\end{aligned}
$$

with $k_{p}=\frac{p \pi}{N}$. Solving these relations yields:

$$
\begin{aligned}
& \vec{X}_{0}(t)=\int_{0}^{t} \vec{\eta}_{0}\left(t^{\prime}\right) \mathrm{d} t^{\prime}+\vec{X}_{0}(0) \\
& \vec{X}_{p}(t)=\vec{X}_{p}(0) \exp \left[-k_{p}^{2} t\right]+\int_{0}^{t} \mathrm{~d} t^{\prime} \exp \left[-k_{p}^{2}\left(t-t^{\prime}\right)\right] \vec{\eta}_{p}\left(t^{\prime}\right)
\end{aligned}
$$

The properties of the noise modes,

$$
\begin{aligned}
\left\langle\vec{\eta}_{p}(t)\right\rangle & =\overrightarrow{0} \\
\left\langle\eta_{\alpha, p}(t) \eta_{\beta, q}\left(t^{\prime}\right)\right\rangle & =\frac{1+\delta_{p, 0}}{N} \delta_{\alpha, \beta} \delta_{p, q} \delta\left(t-t^{\prime}\right),
\end{aligned}
$$

can be obtained with the help of the inverse transformation

$$
\begin{aligned}
\vec{X}_{p} & =\frac{1}{N} \int_{0}^{N} \vec{R}_{h}(n, t) \cos \left(\frac{p \pi n}{N}\right) \mathrm{d} n \\
\vec{\eta}_{p} & =\frac{1}{N} \int_{0}^{N} \vec{\eta}(n, t) \cos \left(\frac{p \pi n}{N}\right) \mathrm{d} n
\end{aligned}
$$


Alternatively the solution $\vec{R}(n, t)$ can be given using the Green's function $G\left(n, t \mid n^{\prime}, t^{\prime}\right)[37]$ :

$$
G\left(n, t \mid n^{\prime}, t^{\prime}\right)=\frac{1}{N}+\frac{2}{N} \sum_{p=1}^{\infty} \cos \left(\frac{p \pi n}{N}\right) \cos \left(\frac{p \pi n^{\prime}}{N}\right) \exp \left[-\left(\frac{p \pi}{N}\right)^{2}\left(t-t^{\prime}\right)\right]
$$

which obeys the von Neumann boundary condition. $\vec{R}(n, t)$ can be written in the form:

$$
\vec{R}(n, t)=\int_{0}^{t} \mathrm{~d} t^{\prime} \int_{0}^{N} \mathrm{~d} n^{\prime} G\left(n, t \mid n^{\prime}, t^{\prime}\right) \vec{f}\left(n^{\prime}, t^{\prime}\right)+\int_{0}^{N} \mathrm{~d} n^{\prime} G\left(n, t \mid n^{\prime}, 0\right) \vec{R}(n, 0) .
$$

\subsection{Observables}

\subsubsection{Center of mass and end-to-end vector}

The simplest observable to calculate is the position of the center of mass, which for the discrete chain is closely connected to the dynamics of the zero ${ }^{\text {th }}$ mode:

$$
\vec{R}_{\text {com }}(t)=\frac{1}{N+1} \sum_{n=0}^{N} \vec{R}_{n}(t)=\frac{1}{\sqrt{N+1}} \vec{X}_{0}(t)
$$

Let us calculate the end-to-end vector defined as the vector connecting the base to the tip of the polymer. The position of the tip and base monomers can be calculated from Eq.(2.37). The end-to-end vector reads:

$$
\vec{R}_{e e}:=\vec{R}_{N}-\vec{R}_{0}=\sqrt{\frac{2}{N+1}} \sum_{p=1}^{N} \vec{X}_{p}(t)\left\{\cos \left[\frac{p \pi(2 N+1)}{2(N+1)}\right]-\cos \left[\frac{p \pi}{2(N+1)}\right]\right\} .
$$

This equation can be simplified using $\cos (a-b)-\cos (a+b)=2 \sin (a) \sin (b)$ as

$$
\vec{R}_{e e}(t)=-2 \sqrt{\frac{2}{N+1}} \sum_{p=o d d}^{N} \vec{X}_{p}(t) \cos \left(\frac{p \pi}{2(N+1)}\right) .
$$

The time correlation of the end-to-end vector can be calculated in terms of the time correlation of the modes:

$$
\left\langle\vec{R}_{e e}\left(t_{1}\right) \cdot \vec{R}_{e e}\left(t_{2}\right)\right\rangle=\frac{8}{N+1} \sum_{p, q=o d d} \cos \left(\frac{p \pi}{2(N+1)}\right) \cos \left(\frac{q \pi}{2(N+1)}\right)\left\langle\vec{X}_{p}\left(t_{1}\right) \cdot \vec{X}_{q}\left(t_{2}\right)\right\rangle .
$$


The correlation of the modes can be obtained as:

$$
\begin{aligned}
\left\langle\vec{X}_{p}\left(t_{1}\right) \cdot \vec{X}_{q}\left(t_{2}\right)\right\rangle & \\
& =\int_{0}^{t_{1}} \int_{0}^{t_{2}} e^{-k_{p}^{2}\left(t_{1}-t^{\prime}\right)} e^{-k_{q}^{2}\left(t_{2}-t^{\prime \prime}\right)}\left\langle\vec{\eta}_{p}\left(t^{\prime}\right) \cdot \vec{\eta}_{q}\left(t^{\prime \prime}\right)\right\rangle \\
& =\frac{3}{N} \delta_{p, q} \int_{0}^{t_{1}} e^{-k_{q}^{2}\left(t_{2}-t^{\prime \prime}\right)} e^{-k_{p}^{2}\left(t_{1}-t^{\prime}\right)}
\end{aligned}
$$

and therefore:

$$
\left\langle\vec{X}_{p}\left(t_{1}\right) \cdot \vec{X}_{q}\left(t_{2}\right)\right\rangle=\frac{3 \delta_{p, q}}{N\left(k_{p}^{2}+k_{q}^{2}\right)} e^{-k_{q}^{2}\left(t_{2}-t_{1}\right)}
$$

Consequently the end-to-end distance autocorrelation can be calculated as:

$$
\left\langle\vec{R}_{e e}\left(t_{1}\right) \cdot \vec{R}_{e e}\left(t_{2}\right)\right\rangle=\frac{24}{N} \sum_{p=o d d} \frac{e^{-k_{p}^{2}\left(t_{1}-t_{2}\right)}}{k_{p}^{2}} .
$$

For large $t_{1}-t_{2}$ this series is dominated by:

$$
\left\langle\vec{R}_{e e}\left(t_{1}\right) \cdot \vec{R}_{e e}\left(t_{2}\right)\right\rangle \rightarrow \frac{24 N}{\pi^{2}} e^{-\left(\frac{\pi}{N}\right)^{2}\left(t_{1}-t_{2}\right)} .
$$

\subsubsection{Segmental motion: short- and long- time Behavior}

Let us consider the dynamics of single monomers on a free Rouse chain. Here we focus on the mean squared displacement of the base point located at $n=0$. We will discuss the behaviour of this point for $t \ll \tau_{R}$ (short time regime) and $t \gg \tau_{R}$ (long time regime).

We can express the dynamics of each of the monomers in terms of the Green's function. Therefore we first approximate the limiting behaviour of the Green's function and then use this approximated Green's function in our calculations.

1. $t \ll \tau_{R}$ : To approximate the Green's function of Eq.(2.59) for the short time regimes, we can use the following form of this Green's function, which can be obtained by the method of images[37]:

$$
G\left(n, t \mid n^{\prime}, t^{\prime}\right)=\frac{1}{2 \sqrt{\pi\left(t-t^{\prime}\right)}} \sum_{p=-\infty}^{+\infty}\left\{\exp \left[-\frac{\left(n-n^{\prime}+2 p N\right)^{2}}{4\left(t-t^{\prime}\right)}\right]+\exp \left[-\frac{\left(n+n^{\prime}+2 p N\right)^{2}}{4\left(t-t^{\prime}\right)}\right]\right\}
$$


This form is particularly convenient for short time approximations. It is easily read off that for large $N$, the short time regime is dominated by the $p=0$ term,

$$
G\left(n, t \mid n^{\prime}, t^{\prime}\right) \rightarrow \frac{1}{2 \sqrt{\pi\left(t-t^{\prime}\right)}}\left\{\exp \left[-\frac{\left(n-n^{\prime}\right)^{2}}{4\left(t-t^{\prime}\right)}\right]+\exp \left[-\frac{\left(n+n^{\prime}\right)^{2}}{4\left(t-t^{\prime}\right)}\right]\right\} .
$$

We note that this result could also be obtained from Eq.(2.59) by converting the infinite sum over $p$ to an integral over a continuous variable $p$. Note that the approximated Green's function does not obey the von Neumann boundary conditions at $n=N$ :

$$
\left.\partial_{n}\right|_{n=N} G\left(n, t \mid n^{\prime}, t^{\prime}\right)=-\frac{\left(N+n^{\prime}\right) \exp \left[-\frac{\left(N+n^{\prime}\right)^{2}}{4\left(t-t^{\prime}\right)}\right]+\left(N-n^{\prime}\right) \exp \left[-\frac{\left(N-n^{\prime}\right)^{2}}{4\left(t-t^{\prime}\right)}\right]}{4 \sqrt{\pi\left(t-t^{\prime}\right)^{3}}}
$$

However, the misfit at $n=N$ typically (i.e. for $n^{\prime}$ a finite fraction of $N$ ) decays as $\left(t-t^{\prime}\right)^{3 / 2} \exp \left[-N^{2} / 4\left(t-t^{\prime}\right)\right]$.

Now using this Green's function we can easily calculate $\left\langle\vec{R}^{2}(n, t)\right\rangle$. For simplicity we consider $n=0$, which we will use in later discussions:

$$
\vec{R}(0, t)=\int_{t_{0}}^{t} \mathrm{~d} t^{\prime} \int_{0}^{N} \mathrm{~d} n^{\prime} G\left(0, t \mid n^{\prime}, t^{\prime}\right) \vec{f}\left(n^{\prime}, t^{\prime}\right)+\int_{n^{\prime}} \mathrm{d} n^{\prime} \vec{R}\left(n^{\prime}, t_{0}\right) G\left(n, t \mid n^{\prime}, t_{0}\right) .
$$

Here we have dropped the term which originated from initial condition which has decayed on the intermediate time scale considered here. Consequently:

$$
\begin{aligned}
\left\langle R^{2}(0, t)\right\rangle \rightarrow & \int_{t_{0}}^{t} \mathrm{~d} t_{1}^{\prime} \int_{t_{0}}^{t} \mathrm{~d} t_{2}^{\prime} \int_{0}^{N} \mathrm{~d} n_{1}^{\prime} \int_{0}^{N} \mathrm{~d} n_{2}^{\prime} \\
& \frac{6 \delta\left(n_{1}^{\prime}-n_{2}^{\prime}\right) \delta\left(t_{1}^{\prime}-t_{2}^{\prime}\right)}{\sqrt{\pi\left(t-t_{1}^{\prime}\right)} \sqrt{\pi\left(t-t_{2}^{\prime}\right)}} e^{-\frac{n_{1}^{\prime 2}}{4\left(t-t_{1}^{\prime}\right)}} e^{-\frac{n_{2}^{\prime 2}}{4\left(t-t_{2}^{\prime}\right)}}
\end{aligned}
$$

We can now perform the integrals over the variables $t_{2}^{\prime}$ and $n_{2}^{\prime}$ which yields:

$$
\left\langle\vec{R}^{2}(0, t)\right\rangle \rightarrow 6 \int_{t_{0}}^{t} \mathrm{~d} t_{1}^{\prime} \int_{0}^{N} \mathrm{~d} n_{1}^{\prime} \frac{e^{-\frac{n_{1}^{\prime 2}}{2\left(t-t_{1}^{\prime}\right)}}}{\pi\left(t-t_{1}^{\prime}\right)}
$$

Integration over $n_{1}^{\prime}$ results in:

$$
\left\langle\vec{R}^{2}(0, t)\right\rangle \rightarrow 3 \int_{t_{0}}^{t} \frac{\operatorname{erf}\left(\frac{N}{\sqrt{2\left(t-t_{1}^{\prime}\right)}}\right)}{\sqrt{2 \pi\left(t-t_{1}^{\prime}\right)}}
$$


Note, that we are in the short time regime, i.e. $t \ll N^{2}$. Therefore we can use the asymptotic expansion of the error function which is:

$$
\operatorname{erf}(x) \rightarrow 1-\frac{e^{-x^{2}}}{x \sqrt{\pi}}+\mathcal{O}\left(\frac{e^{-x^{2}}}{x^{2}}\right)
$$

and eventually we obtain:

$$
\left\langle R^{2}(0, t)\right\rangle \rightarrow 3 \sqrt{\frac{\left(t-t_{0}\right)}{2}} .
$$

The monomer thus moves sub-diffusively over a period of time, which grows with chain length. This derivation is easily generalized to any other monomers in the chain.

2. $t \gg \tau_{R}$ For times much larger than the Rouse time, all modes with $p>0$ have relaxed to zero. In this time regime, we can thus approximate the Green's function Eq.(2.59) as:

$$
G\left(n, t \mid n^{\prime}, t^{\prime}\right) \rightarrow \frac{1}{N}
$$

Now the mean squared displacement of the base monomer (and also any other monomer) becomes:

$$
\left\langle R^{2}(0, t)\right\rangle \rightarrow \int_{t_{0}}^{t} \mathrm{~d} t_{1}^{\prime} \int_{t_{0}}^{t} \mathrm{~d} t_{2}^{\prime} \int_{0}^{N} \mathrm{~d} n_{1}^{\prime} \int_{0}^{N} \mathrm{~d} n_{2}^{\prime} \frac{6}{N^{2}} \delta\left(t_{1}^{\prime}-t_{2}^{\prime}\right) \delta\left(n_{1}^{\prime}-n_{2}^{\prime}\right)=\frac{6 t}{N}
$$

Thus the motion of a monomer crosses over from subdiffusive behaviour to diffusive behaviour with increasing time.

\subsubsection{Tension dynamics}

In a discrete chain the force exerted on the $n^{t h}$ monomer by the $(n-1)^{t h}$ monomer is given by:

$$
-\vec{b}_{n}(t)=-\vec{R}_{n}(t)+\vec{R}_{n-1}(t)
$$

Here we have used the dimensionless variables introduced in Section 2.1.3. Correspondingly, in the continuous chain, the line tension is related to the bond vectors as:

$$
-\vec{b}(n, t)=-\partial_{n} \vec{R}(n, t) .
$$


All the monomer positions $\vec{R}_{n}$ can be reconstructed from the bond variables $\vec{b}_{n}$ and the center-of-mass position $\vec{R}_{\text {com }}$. To see this we start from:

$$
\vec{R}(n, t)=\vec{R}(0, t)+\sum_{i=1}^{n} \vec{b}(i, t)
$$

and insert into $\vec{R}_{\text {com }}=(1 / N+1) \sum_{n} \vec{R}_{n}$. This leads to:

$$
\vec{R}(0, t)=\vec{R}_{\text {com }}-\sum_{n=1}^{N}\left(1-\frac{n}{N+1}\right) \vec{b}(n, t) .
$$

In the continuous version the sum becomes an integral $R(0, t)=R_{\text {com }}-\int_{0}^{N}(1-$ $\left.\frac{n}{N}\right) b\left(n^{\prime}, t\right) \mathrm{d} n^{\prime}$ and therefore:

$$
\vec{R}(n, t)=\int_{0}^{n} \vec{b}\left(n^{\prime}, t\right) \mathrm{d} n^{\prime}-\int_{0}^{N}\left(1-\frac{n^{\prime}}{N}\right) \vec{b}\left(n^{\prime}, t\right) \mathrm{d} n^{\prime}+\vec{R}_{c o m}
$$

The dynamics of the fixed-length polymer chain may alternatively be described in terms of the bond vectors. According to Eq.(2.76), we obtain the evolution equation for the bonds by differentiating both sides of Eq.(2.29) with respect to $n$ :

$$
\partial_{t} \vec{b}(n, t)=\partial_{n}^{2} \vec{b}+\partial_{n} \vec{f}
$$

For a free chain the von Neumann boundary conditions for $\vec{R}$ change to Dirichlet type for bond vectors. Such a transformation of a boundary condition of von Neumann to Dirichlet can be extremely helpful in analyzing growing chains.

Note that the regulatity properties of $\vec{b}$ are weaker than those of $\vec{R}$ due to the derivative of spatial white noise driving the system. However, this causes no problem as can be seen by representing the solution to Eq.(2.80) in terms of Green's functions $G_{D}$ obeying homogeneous Dirichlet boundary conditions:

$$
\vec{b}(n, t)=\int_{0}^{N} \vec{b}\left(n^{\prime}, 0\right) G_{D}\left(n, n^{\prime}, t\right) \mathrm{d} n^{\prime}+\int_{0}^{t} \int_{0}^{N} G_{D}\left(n, n^{\prime}, t-t^{\prime}\right) \partial_{n^{\prime}} \vec{f}\left(n^{\prime}, t^{\prime}\right) \mathrm{d} n^{\prime} \mathrm{d} t^{\prime} .
$$

The derivative of the noise can be transferred to the Green's function using partial integration. This yields:

$$
\begin{aligned}
\vec{b}(n, t)= & \int_{0}^{t} \int_{0}^{N} \partial_{n^{\prime}}\left[G_{D}\left(n, t \mid n^{\prime}, t^{\prime}\right) \vec{f}\left(n^{\prime}, t^{\prime}\right)\right] \\
& -\int_{0}^{t} \int_{0}^{N} \vec{f}\left(n^{\prime}, t^{\prime}\right) \partial_{n^{\prime}} G_{D}\left(n, t \mid n^{\prime}, t^{\prime}\right) \mathrm{d} n^{\prime} \mathrm{d} t^{\prime}
\end{aligned}
$$


The first term on the right hand side vanishes due to the boundary condition for $G_{D}$ and thus:

$$
\vec{b}(n, t)=-\int_{0}^{t} \int_{0}^{N} \vec{f}\left(n^{\prime}, t^{\prime}\right) \partial_{n^{\prime}} G_{D}\left(n, t \mid n^{\prime}, t^{\prime}\right) \mathrm{d} n^{\prime} \mathrm{d} t^{\prime} .
$$

To solve the dynamical equation for $\vec{b}$, we have to compute the derivative of the Green's function with respect to $n^{\prime}$ :

$$
\partial_{n^{\prime}} G_{D}\left(n, t \mid n^{\prime}, t^{\prime}\right)=\frac{2}{N} \sum_{p=1}^{\infty} k_{p} \sin \left(k_{p} n\right) \cos \left(k_{p} n^{\prime}\right) \exp \left[-k_{p}^{2}\left(t-t^{\prime}\right)\right]
$$

Let us illustrate how to use Eq.(2.82) to calculate statistical properties of the conformation. The end-to-end vector is particularly easy to express via bonds in the form:

$$
\begin{aligned}
R_{e e}(t) & =\int_{0}^{N} b(n, t) \mathrm{d} n \\
& =\frac{2}{N} \sum_{p=1}^{\infty}\left[(-1)^{p}-1\right] \int_{0}^{N} \mathrm{~d} n^{\prime} \cos \left(k_{p} n^{\prime}\right) \int_{0}^{t} \exp \left[-k_{p}^{2}\left(t-t^{\prime}\right)\right] \vec{f}\left(n^{\prime}, t^{\prime}\right) \mathrm{d} t^{\prime}
\end{aligned}
$$

and this leads to the end-to-end vector autocorrelation:

$$
\begin{aligned}
\left\langle R_{e e}(t) \cdot R_{e e}\left(t_{0}\right)\right\rangle & =\frac{4}{N^{2}} \sum_{p, p^{\prime}=1}^{\infty}\left[(-1)^{p}-1\right]\left[(-1)^{p^{\prime}}-1\right] \int_{n^{\prime}, m^{\prime}=0}^{n^{\prime}, m^{\prime}=N} \mathrm{~d} n^{\prime} \mathrm{d} m^{\prime} \cos \left(k_{p} n^{\prime}\right) \cos \left(k_{p^{\prime}} m^{\prime}\right) \\
& \times \int_{t^{\prime}=0}^{t^{\prime}=t} \int_{t_{1}^{\prime}=0}^{t_{1}^{\prime}=t_{0}} \exp \left[-k_{p}^{2}\left(t-t^{\prime}\right)\right] \exp \left[-k_{p^{\prime}}^{2}\left(t_{0}-t_{1}^{\prime}\right)\right]\left\langle\vec{f}\left(n^{\prime}, t^{\prime}\right) \cdot \vec{f}\left(m^{\prime}, t_{1}^{\prime}\right)\right\rangle \mathrm{d} t^{\prime} \mathrm{d} t_{1}^{\prime} .
\end{aligned}
$$

Hence if we suppose $0<t_{0}<t$ we can first perform the integration over $t^{\prime}$ and then over $t_{1}^{\prime}$ :

$$
\begin{aligned}
\left\langle R_{e e}(t) \cdot R_{e e}\left(t_{0}\right)\right\rangle & =\frac{4}{N^{2}} \sum_{p, p^{\prime}=1}^{\infty}\left[(-1)^{p}-1\right]\left[(-1)^{p^{\prime}}-1\right] \\
& \times 6 \frac{N}{2} \delta_{p, p^{\prime}} \int_{t_{1}^{\prime}=0}^{t_{1}^{\prime}=t_{0}} \exp \left[-k_{p}^{2}\left(t-t_{1}^{\prime}\right)\right] \exp \left[-k_{p^{\prime}}^{2}\left(t_{0}-t_{1}^{\prime}\right)\right] \mathrm{d} t_{1}^{\prime} .
\end{aligned}
$$

Performing the sum over $p^{\prime}$ leads to:

$$
\begin{gathered}
\left\langle R_{e e}(t) \cdot R_{e e}\left(t_{0}\right)\right\rangle=\frac{16}{N^{2}} \sum_{p=o d d}^{\infty} 6 \frac{N}{2} \exp \left[-k_{p}^{2}\left(t+t_{0}\right)\right] \int_{t_{1}^{\prime}=0}^{t_{1}^{\prime}=t_{0}} \exp \left[2 k_{p}^{2} t_{1}^{\prime}\right] \mathrm{d} t_{1}^{\prime} \\
\approx \frac{24 N}{\pi^{2}} \exp \left[-k_{p}^{2}\left(t-t_{0}\right)\right]
\end{gathered}
$$


which exactly reproduces the result of Eq.(2.67). Finally, let us stress that all discussions and results of this section are restricted to chains of fixed length $N$. In the next section, we continue our discussion with a description of the dynamics of chemical reactions, which change the chain length.

\subsection{Polymerization reactions}

\subsubsection{Markov model of chemical reactions}

We start from the Markovian description of chemical reactions. Let us consider a wellstirred mixture of $K$ molecular species inside a reaction chamber of a fixed volume. These species may take part in $M$ different kinds of chemical reactions $\mathcal{R}_{1}, \mathcal{R}_{2}, \cdots, \mathcal{R}_{M}$. In a single reaction $\mathcal{R}_{\mu}$ the population of the reactants $Z=\left\{Z_{1}, Z_{2}, \cdots, Z_{K}\right\}$ change according to:

$$
\mathcal{R}_{\mu}: Z_{i} \rightarrow Z_{i}+v_{i, \mu}
$$

Here $v_{i, \mu}$ is the $i^{t h}$ component of the vector $\boldsymbol{V}_{\mu}$. This vector represents the change in the population of the species due to the reaction $\mathcal{R}_{\mu}$. Note that each reaction appears stochastically with the rate $a_{\mu}(\boldsymbol{Z})$ (also called "propensity"). The probability $P(\boldsymbol{Z}, t)$ can be determined from the Master equation:

$$
\dot{P}(\boldsymbol{Z}, t)=\sum_{\mu=1}^{M}\left[a_{\mu}\left(\boldsymbol{Z}-\boldsymbol{V}_{\mu}\right) P\left(\boldsymbol{Z}-\boldsymbol{V}_{\mu}, t\right)-a_{\mu}(\boldsymbol{Z}) P(\boldsymbol{Z}, t)\right],
$$

and the initial condition $P(\boldsymbol{Z}, 0)=P_{0}(\boldsymbol{Z})$. On the well-stirred conditions the rates $a_{\mu}$ can be written in the form:

$$
a_{\mu}(\boldsymbol{Z})=\gamma_{\mu} h_{\mu}(\boldsymbol{Z})
$$

where $\gamma_{\mu}$ is the rate constant of the reaction $\mathcal{R}_{\mu}$ and $h_{\mu}$ is the number of distinct combinations of the educts of $\mathcal{R}_{\mu}$ per volume. For instance, in a bimolecular reaction $\mathrm{S}_{1}+\mathrm{S}_{2} \longrightarrow \mathrm{S}_{3}$, there are $Z_{1} \cdot Z_{2}$ combinations of the reactants $S_{1}$ and $S_{2}$ which are all equally probable under well-stirred conditions. From the chemical Master equation we obtain ordinary differential equations for the average concentrations $\langle\boldsymbol{Z}\rangle / V$, called the rate equations. Note that the rate equations are in general not a closed set of equations to determine the $\langle\boldsymbol{Z}\rangle$ with the important exception of propensities linear in $\boldsymbol{Z}$.

From the Markovian description of chemical reactions we can find the distribution of waiting times. By definition, $p_{w}(\tau, \mu \mid \boldsymbol{Z}, t)$ is the probability that the next chemical reaction is an $\mathcal{R}_{\mu}$ reaction and will occur at time $t+\tau{ }^{1}$. The total rate, that an

\footnotetext{
${ }^{1}$ In fact, in the infinitesimal time interval $[t+\tau, t+\tau+\mathrm{d} \tau)$
} 
interaction happens is $\Gamma:=\sum_{\mu} a_{\mu}$. Therefore the probability that in a short time interval $\mathrm{d} \tau$ no interaction occurs is $1-\Gamma \mathrm{d} \tau$. Thus, if one splits the interval $(t, t+\tau)$ into many sub-intervals with length $\mathrm{d} \tau$, then in the limit $\mathrm{d} \tau \rightarrow 0$ the probability of no reaction can be written as $\propto e^{-\Gamma \tau}$. Thus $p_{w}$ becomes:

$$
p_{w}(\tau, \mu \mid \boldsymbol{Z}, t)=a_{\mu}(\boldsymbol{Z}) \exp (-\Gamma \tau) .
$$

\subsubsection{Chain growth reactions}

Let us now consider the special case of reversible chain-growth polymerization reactions for which monomers can bind or unbind (one by one) to an active end of the chain which we will choose as the tip further on. We will assume here that the other end of the polymer is blocked, i.e. it can neither bind nor loose monomers. We restrict our discussions to this simple case, although many of our results can also be generalised to two active ends.

To describe the dynamics of such a process, we first have to specify the reacting species in the system. A living polymerization requires just two different species:

1. $\mathrm{M}_{c}$ : Monomers, which make up the chain.

2. $\mathrm{M}_{f}$ : Free Monomers which are not attached to the polymer.

As mentioned in the previous section, we represent the chemical state of this system as the number of the molecules of these species:

$$
\boldsymbol{Z}:=\left[\begin{array}{l}
Z_{c} \\
Z_{f}
\end{array}\right]
$$

For a reversible chain-growth polymerization, there are two kinds of chemical reactions in the system:

1. Polymerization:

$$
\mathrm{M}_{\mathrm{f}} \stackrel{\gamma_{p}}{\longrightarrow} \mathrm{M}_{\mathrm{C}}
$$

Note that this chemical notation is incomplete because it leaves out the polymer. The rate $\gamma_{p}$ depends on the functionality of the chain bound species. For our purposes, we assume that the $\mathrm{M}_{c}$ are bi-functional, but that the base $(n=0)$ monomer (resulting from the starting process of polymerization) is capped and inactive. Thus the chain changes its length only at the tip. 
Therefore, according to Eq.(2.87):

$$
h_{p}=\rho_{f}
$$

where $\rho_{f}=Z_{f} / V$ is the concentration of free monomers in the system. Note that here we assumed that there is only one chain (and consequently one reactive tip) in the system. In this case we define the stochastic chain length as:

$$
\hat{N}:=Z_{c}
$$

As will be seen, in building our model for polymerizing chains the fixed length $N$ of a Rouse chain introduced in Section 2.1 is substituted by this stochastic length $\hat{N}$. The propensity of such a reaction, according to Eq.(2.87) is thus:

$$
a_{p}=\gamma_{p} \rho_{f}
$$

As the polymer changes its conformation in space, the tip will move around and may leave the well-stirred compartment $V$, entering other compartments with different free monomer concentrations. This is a possible coupling between conformational and chemical dynamics, which is absent if the whole volume, visited by the polymer always remains well-stirred.

In each reaction, the numbers of free and bound monomers change by 1 , so

$$
\boldsymbol{V}_{p}:=\left[\begin{array}{l}
+1 \\
-1
\end{array}\right]
$$

2. Depolymerization:

$$
\mathrm{M}_{\mathrm{c}} \stackrel{\gamma_{d}}{\longrightarrow} \mathrm{M}_{\mathrm{f}}
$$

Here we have used the index $d$ for the depolymerization process. This is assumed to be a mono-molecular and for such a reaction the propensity reads:

$$
a_{d}=\gamma_{d}
$$

The jump vector of this reaction reads:

$$
\boldsymbol{V}_{d}:=\left[\begin{array}{l}
-1 \\
+1
\end{array}\right]
$$

We consider the relative fluctuations in the number of free monomers due to the polymerization/depolymerization processes as negligible, i.e. we always replace $\rho_{f}$ by $\left\langle\rho_{f}\right\rangle$, if the system is in contact with a reservoir of free monomers. This seems to be a realistic 
assumption, especially in cases where only a small number of initiated polymers (like here) are introduced. Such a situation happens for instance in a typical polymerization of actin filaments, where the number of G-actins in a $10-50$ micromolar environment is about $10^{17}$ particles per litre, while the fluctuations due to the reversible polymerization is about 10 monomers per second. In many situations, the concentration of free monomers is controlled by some external mechanism, which either keeps it fixed or imposes an explicit time dependence $\rho_{f}(t)$.

Given the time-dependent deterministic concentration $\rho_{f}(t)$, the evolution of the population of the chain length can be determined by the Master equation:

$$
\dot{P}(N, t)=a_{p}(t) P(N-1, t)+a_{d} P(N+1, t)-\left(a_{p}(t)+a_{d}\right) P(N, t) .
$$

The first moment of $\hat{N}$ can be obtained by multiplying both sides of the Master equation (2.98) by $N$ and sum over $N$ :

$$
\frac{\mathrm{d}\langle\hat{N}\rangle}{\mathrm{d} t}=a_{p}(t) \sum_{N=1}^{\infty} N P(N-1, t)+a_{d} \sum_{N=1}^{\infty} N P(N+1, t)-\left(a_{p}(t)+a_{d}\right) \sum_{N=1}^{\infty} N P(N, t) .
$$

which results in the rate equation for the chain length:

$$
\frac{\mathrm{d}\langle\hat{N}\rangle}{\mathrm{d} t}=a_{p}-a_{d}
$$

This equation can simply be solved as $\langle\hat{N}\rangle=\int_{t_{0}}^{t}\left[a_{p}\left(t^{\prime}\right)-a_{d}\right] \mathrm{d} t^{\prime}$.

To estimate the relative fluctuations in the polymer length we need to calculate the second moment of $\hat{N}$ by multiplying both sides of Eq.(2.98) by $N^{2}$ :

$\frac{\mathrm{d}\left\langle\hat{N}^{2}\right\rangle}{\mathrm{d} t}=a_{p}(t) \sum_{N=1}^{\infty} N^{2} P(N-1, t)+a_{d} \sum_{N=1}^{\infty} N^{2} P(N+1, t)-\left(a_{p}(t)+a_{d}\right) \sum_{N=1}^{\infty} N^{2} P(N, t)$.

Reindexing the summation appropriately yields:

$$
\left\langle\hat{N}^{2}\right\rangle-\langle\hat{N}\rangle^{2}=\int_{t_{0}}^{t}\left[a_{p}\left(t^{\prime}\right)+a_{d}\right] \mathrm{d} t^{\prime} .
$$

The relative fluctuations can thus be estimated as:

$$
\frac{\left\langle\hat{N}^{2}\right\rangle-\langle\hat{N}\rangle^{2}}{\langle\hat{N}\rangle^{2}}=\frac{\int\left[a_{p}(t)+a_{d}\right] \mathrm{d} t}{\left(\int\left[a_{p}(t)-a_{d}\right] \mathrm{d} t\right)^{2}} .
$$

In this equation if we neglect $a_{d}$ compared to $a_{p}$-which is justified when a large concentration of free monomers is provided- we can approximate the relative fluctuations 
as:

$$
\frac{\left\langle\hat{N}^{2}\right\rangle-\langle\hat{N}\rangle^{2}}{\langle\hat{N}\rangle^{2}} \approx \frac{1}{\left(t-t_{0}\right) \gamma_{p} \bar{\rho}_{f}(t)}
$$

where $\bar{\rho}_{f}(t)$ is introduced as the time average of the concentration of the free monomers during $t_{0}$ and $t$. For a power-law propensity $a_{p}(t) \sim t^{\alpha-1}$ we have

$$
\frac{\left\langle\hat{N}^{2}\right\rangle-\langle\hat{N}\rangle^{2}}{\langle\hat{N}\rangle^{2}} \sim t^{-\alpha}
$$

. Therefore for $\alpha>0$, this relative fluctuations decreases in time and thus in the asymptotically long time the chemical noise can be neglected (see Section 2.5.4).

To simulate the reactions we will use the waiting time probability $p_{w}(\tau, \mu \mid t)$, where $\mu \in\{p, d\}$. Note that to keep the notation simple we dropped the dependence of the waiting time on $Z_{f}$. In the following, we will derive the waiting time distribution for the case, where the propensities are explicitly time dependent. To calculate the waiting time distribution we first partition the time interval $[t, t+\tau)$ into small time intervals with length $\Delta t$. According to the definition of propensity, the probability that neither a polymerization nor depolymerization reaction happens between the small interval $\left[t_{i}, t_{i}+\right.$ $\Delta t)$ is $1-\left(a_{p}(t)+a_{d}\right) \Delta t$. The probability that no reaction takes place within the interval $[t, t+\tau)$ is thus the joint probability of such events:

$$
\tilde{p}=\prod_{i}\left[1-\left(a_{p}\left(t_{i}\right)+a_{d}\left(t_{i}\right)\right) \Delta t\right] \approx \prod_{i} e^{-\left[a_{p}\left(t_{i}\right)+a_{d}\left(t_{i}\right)\right] \Delta t}=e^{-\sum_{i}\left[a_{p}\left(t_{i}\right)+a_{d}\left(t_{i}\right)\right] \Delta t}
$$

and thus for the limit $\Delta t \rightarrow 0$ we have $\tilde{p}=e^{-\int_{t}^{t+\tau}\left[a_{p}\left(t^{\prime}\right)+a_{d}\right] \mathrm{d} t^{\prime}}$. The waiting time probability is the joint probability of no reaction during $[t, t+\tau)$ and reacting during $[t+\tau, t+\tau+\mathrm{d} \tau)$. Therefore:

$$
p_{w}(\tau, \mu \mid t)=a_{\mu}(t+\tau) e^{-\int_{t}^{t+\tau}\left[a_{p}\left(t^{\prime}\right)+a_{d}\right] \mathrm{d} t^{\prime}}
$$

Now we are ready to establish our model of dynamical behaviour of a growing polymer chain by combining the two aspects of the dynamics: 1) the conformational dynamics of the polymer, which we described by the Rouse model (see Section 2.1 ) and 2) the dynamics of the chain length $\hat{N}$, which is described by the stochastic model introduced in this section. 


\subsection{Model of conformational dynamics of a living Rouse chain}

\subsubsection{Couplings between chemical and conformational dynamics}

To introduce our model for growing chains (both in discrete and continuum versions) let us first discuss possible couplings between conformational and chemical dynamics. In particular, we distinguish between:

- Dependence of polymer conformation on chemical reaction: In Section 2.1, we discussed the dependence of the conformational dynamics of the polymer on its length. This length-dependence is an obvious and unavoidable coupling between chemical reactions and conformational dynamics and is the main topic of this thesis. In the literature the chemical processes and the conformational dynamics are both well-studied, but usually kept separate. This may be justified if the time-scales of the two processes differ vastly. But in many important situations this is not the case. For instance, in a typical polymerization of polystyrene where the relaxation time of the chain containing $\sim 10^{4}$ monomers varies in a range between $(1-10) \times 10^{-3}$ seconds[38], new monomers are added to the chain with a rate of $\sim 10^{3}$ monomers per second[17-19]. As another example, actin filaments in living cells which relax on a time scale of about 10 seconds, grow with a rate of $50-500$ monomers per second. In cellular environments, monomers or regulators of reactions are provided in limited amount and degrade or diffuse away from the reaction volume and may lead to decreasing reaction rates[20, 22-25]. We are interested in new phenomena, which originate from this coupling. In the theoretical analysis the coupling is accomplished by updating the dimension of the Rouse matrix (in the discrete model) or by introducing a time varying boundary $0<n<\hat{N}(t)$ in the continuum version of the Rouse model.

- Dependence of chemical reactions on polymer conformation: A possible coupling between the polymer length $\hat{N}$ and the conformational dynamics of the chain has been mentioned in Section 2.4: In a chain-growth polymerization, the active center is considered to be at the tip of the polymer namely $\vec{R}_{t i p}(t)$. If the polymerization is taking place in an inhomogeneous concentration field $\rho_{f}(r, t)$, the propensity $a_{p}$ depends upon the dynamics of the tip position.

Note that the latter coupling always appears in combination with the former type of coupling, and the combination of both will lead to significantly more complex situations and require a much more involved theoretical analysis. Therefore, as a first step, this 
thesis concentrates on the first coupling, which is universally present. This requires the free monomer concentration to be homogeneous and externally controlled.

\subsubsection{Living Rouse chain: discrete model}

Let us number the times of reactions as $t_{i}, i=1,2, \cdots$. For times in between two reactions, $t_{i}<t<t_{i+1}$ the discrete chain evolves as a Rouse chain of length $N_{i}=$ $\hat{N}\left(t_{i}^{+}\right)$, where $t_{i}^{+}$refers to the time immediately after the $i$-th reaction. This part of the process is Markovian and is completely characterized by the transition probability, $P_{\text {Rouse }}\left(\boldsymbol{R}_{N_{i}}, t \mid \boldsymbol{R}_{N_{i}}, t_{i}\right)$ which we will calculate explicitly. The dynamics of the chain length $\hat{N}(t)$ is a simple point process with jumps \pm 1 appearing at the reaction times $t_{i}$. Polymerization reactions are described as compound point processes, which not only change $\hat{N} \rightarrow \hat{N} \pm 1$, but also change the position of the reacting tip. They are characterized by the rates $a_{p}(t)$ and $a_{d}(t)$ of their occurrence and by the probability distributions of the changes of the tip position $P_{\mu}$.

For a polymerization reaction, within the infinitesimal interval $\left[t_{i}, t_{i}^{+}\right)$, a new bead at position $\vec{R}_{\text {new }}$ is added to the tip of the polymer. We consider this process to happen so fast, compared to the dynamics of the whole chain, that the adiabatic approximation is valid. Therefore a new bond $\vec{b}_{n e w}:=\vec{R}_{n e w}-\vec{R}_{\text {tip }}$ with the equilibrium statistics will be added to the tip of the chain placed at $\vec{R}_{t i p}$. The Gaussian probability $P_{b}\left(\vec{R}_{n e w}-\vec{R}_{t i p}\right)$ of this bond can be characterized by its first and second moments:

$$
\begin{aligned}
& \left\langle\vec{b}_{\text {new }}\right\rangle=0 \\
& \left\langle\vec{b}_{\text {new }}^{2}\right\rangle=1
\end{aligned}
$$

where the average is over $P_{b}$. Alternatively we can add the new bond with length $b_{\text {new }}=0$. Then it will equilibrate on the microscopic timescale $\tau_{1}$ given in Eq.(2.45) and for timescales longer than $\tau_{1}$ the tip will perform random steps of equilibrium length $b$.

For a depolymerization reaction, within the interval $\left[t_{i}, t_{i}^{+}\right)$an existing bond at the tip of the polymer dissociates from the chain.

The coupling between conformational changes and chemical reactions can thus be given by the probability densities $P_{\mu}\left(\overrightarrow{\boldsymbol{R}}_{N_{i}}\left(t_{i}^{+}\right) \mid \overrightarrow{\boldsymbol{R}}_{N_{i-1}}\left(t_{i}\right)\right)$ with:

$$
P_{\mu}= \begin{cases}P_{b}\left(\vec{R}_{N_{i}}\left(t_{i}^{+}\right)-\vec{R}_{N_{i-1}}\left(t_{i}\right)\right) \delta^{N_{i-1}}\left(\overrightarrow{\boldsymbol{R}}_{N_{i}}^{\prime}\left(t_{i}^{+}\right)-\overrightarrow{\boldsymbol{R}}_{N_{i-1}}\left(t_{i}\right)\right) & \mu=+1 \\ \delta^{\hat{N}\left(t_{i}^{+}\right)}\left(\overrightarrow{\boldsymbol{R}}_{N_{i}}\left(t_{i}^{+}\right)-\overrightarrow{\boldsymbol{R}}_{N_{i-1}}^{\prime}\left(t_{i}\right)\right) & \mu=-1\end{cases}
$$


where we introduced $\overrightarrow{\boldsymbol{R}}^{\prime}$ as the conformation of the chain, except the tip point. These probability densities will be used in Section 3.1 where we present our algorithm to simulate the discrete model.

A realisation of the complete process is made up of a "chemical path" and a "conformational path". Important quantities specifying the chemical path are given in Table 2.1 and the complete process is visualized in Figure 2.2.

\begin{tabular}{l|l}
\hline Path Specification & Description \\
\hline \hline$M(t)$ & path length: the total number of chemical reactions until time $t$ \\
\hline$\left\{\tau_{i}\right\}_{i=0 \cdots M-1}$ & waiting times: the time between two chemical reactions \\
\hline$\left\{t_{i}\right\}_{i=0 \cdots M}$ & reaction times: the moments at which chemical reactions take place \\
\hline$\left\{\mu_{i}\right\}_{i=0 \cdots M-1}$ & $\begin{array}{l}\mu_{i}=+1: \text { polymerization, } \mu_{i}=-1: \text { depolymerization } \\
\hat{N}(t)\end{array}$ \\
\hline
\end{tabular}

TABLE 2.1: Important quantities corresponding to a chemical path.

In Section 3.1 we present our algorithm to simulate the discrete model.

\subsubsection{Living Rouse chain: continuous model}

To address the problem of growing chains analytically, we use the continuous version of the Rouse model. In the discrete version due to the addition of new monomers during the polymerization process, the number of coupled stochastic equations for beads is constantly growing and therefore the standard approach presented in Section2.2 is no longer applicable. For our analytical purposes, we take advantage of the model in continuum limit.

To set up the corresponding model for growing chains in the continuum limit, we have to couple the conformational dynamics, given in Eq.(2.29) to the chemical dynamics, which enters at the reacting boundary $\vec{R}(\hat{N}(t), t)$. In particular this means that the domain $0<n<\hat{N}(t)$ is changing with time, which turns the fixed-length Rouse dynamics into a moving boundary value problem. 


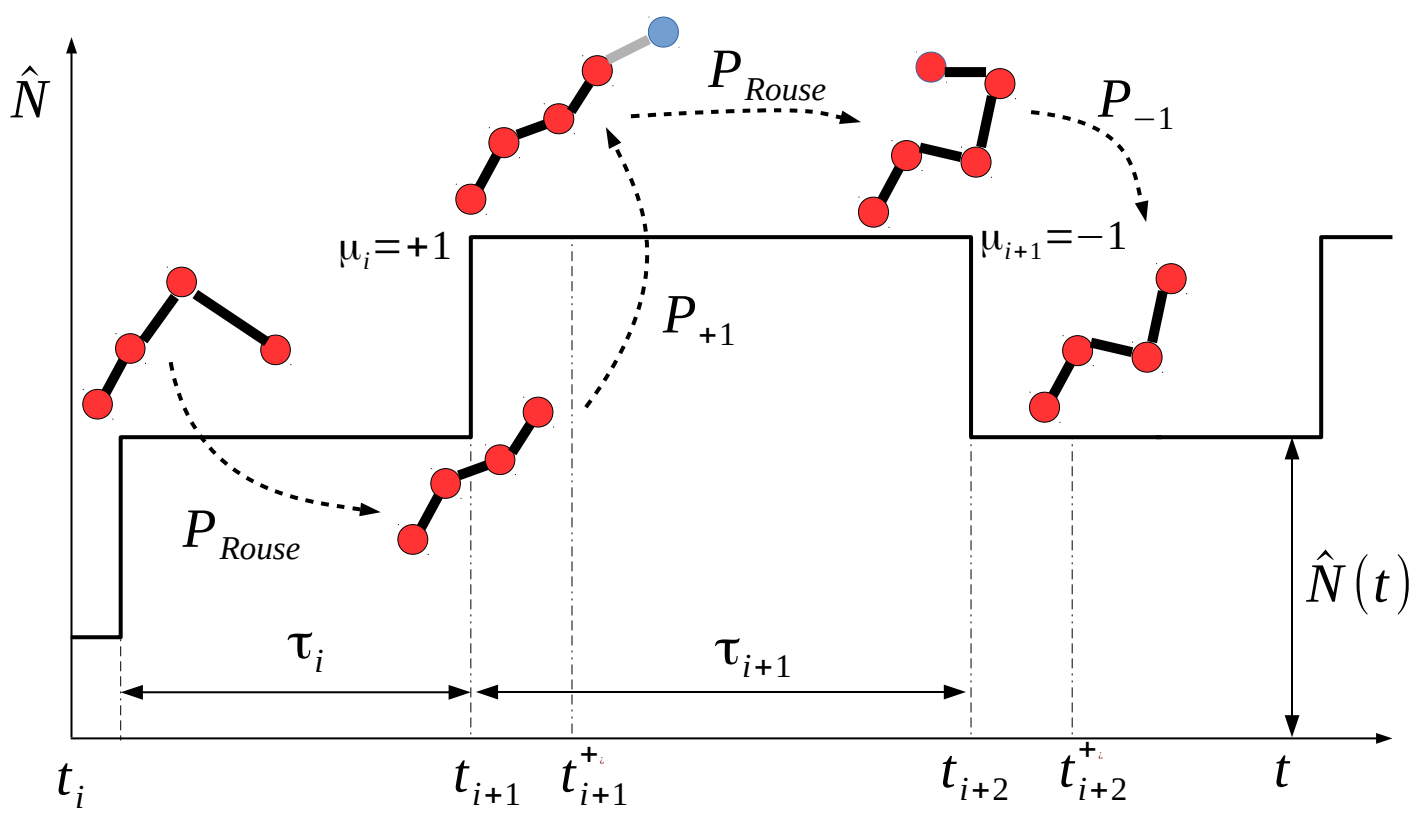

FIGURE 2.2: One realization of a chemical path with $\sum_{i} \mu_{i}=\hat{N}(t)$ and the conformational evolution of a Rouse chain, under such a path: During $\left(t_{i}, t_{i+1}\right]$, the chain evolves according to the Rouse dynamcis. In the infinitesimal time interval $\left[t_{i+1}, t_{i+1}^{+}\right)$, a new segment (is shown in grey) is added to the tip of the chain. Again from $t_{i+1}^{+}$to $t_{i+2}$ the chain evolves as a Rouse chain. In the infinitesimal time interval $\left[t_{i+2}, t_{i+2}^{+}\right)$, an existing segment dissociates from the tip of the polymer. These steps continue until a trajectory of the chain with a final length $\hat{N}(t)$ is completed.

Moving boundary problems are very widespread in various processes: solidification of metals and alloys[39], melting of polymers[40] and heat conduction in a block of ice during freezing/melting[41] are only a few examples, where moving boundary problems are arising. Despite the practical importance of moving boundary problems, only a few special cases have ever been solved exactly[42]. The majority of them can merely be treated numerically. As an additional complication, in our case the movement of the boundary may be stochastic.

Let us now consider the boundary conditions appropriate for a continuously growing chain. we have to include the newly added bonds described in the discrete version. There we have introduced two variants of the growth step, which can be transferred to the continuum limit. On the one hand growth steps with equilibrium length lead to a random walk of the tip, which could be modeled as a Langevin equation of the form $\partial_{N} \vec{R}_{t i p}=\vec{f}_{g}$

and this could be taken as a boundary condition, leading to fluctuating tensions at the tip. On the other hand, inserting new monomers with $b_{\text {new }}=0$ leads to a homogeneous von Neumann boundary condition. Here we chose the second option. In fact, it turns out that the tip still performs a random walk because an added length element $\mathrm{d} N$, inserted 
at the position of the tip, equilibrates on microscopic time scales, thereby leading to a random step of length $b$ (Kuhn length) in space.

Another simplification that we may apply during this thesis is to neglect the chemical noise and consider that the chain length evolves deterministically in time. As mentioned in Section 2.4, there are situations where the relative fluctuations of the chain length due to the stochastic chemical reactions can be neglected. Thus, in the course of this thesis, we replace the stochastic length $\hat{N}$ by the deterministic value $N=\langle\hat{N}(t)\rangle$.

We further assume that the polymerization is irreversible. In other words, we consider the case, that once a monomer binds to the tip, it stays there and no depolymerization reaction occurs.

In summary, our simplified model of a continuous and growing Rouse chain is defined by the evolution equation:

$$
\partial_{t} \vec{R}(n, t)=\partial_{n}^{2} \vec{R}(n, t)+\vec{f}(n, t)
$$

on the time-dependent domain

$$
0 \leq n \leq N(t)
$$

where $N(t)$ is a monotonically increasing function of time. The partial stochastic differential equation given in Eq.(2.105) has to be supplemented by some boundary conditions. For instance, for a free chain:

$$
\begin{aligned}
\left.\partial_{n} \vec{R}\right|_{n=0} & =0 \\
\left.\partial_{n} \vec{R}\right|_{n=N(t)} & =0 .
\end{aligned}
$$

Alternatively, we may study the dynamics of bond variables (or tension) by setting up the evolution equation as:

$$
\partial_{t} \vec{b}(n, t)=\partial_{n}^{2} \vec{b}(n, t)+\partial_{n} \vec{f}(n, t)
$$

For the bond dynamics the boundary conditions corresponding to Eqs.(2.107) and (2.108) are

$$
\begin{aligned}
\vec{b}(0, t) & =0 \\
\vec{b}(N(t), t) & =0
\end{aligned}
$$




\subsubsection{Non-stationary growth}

Throughout this thesis, we restrict our discussions to pure power-law for the propensity function, i.e. $a_{p}(t) \sim t^{\alpha-1}$ with $\alpha>1$. As mentioned in Section 2.4.2, in this case the relative fluctuations of the chain length decrease in time and therefore the chain length growth deterministically without bond as the power-law:

$$
N(t)=\gamma t^{\alpha}
$$

Diffusion of microscopic particles in crowded intracellular environments is often observed to be sub-diffusive[43] and sometimes super-diffusive[44]. One can thus imagine such anomalous transport mechanism for monomers and activators involving in polymerization processes. In these situations, the concentration of free monomers are typically decreasing as a function bounded by power-laws. Figure 2.3 demonstrates schematically a situation, when monomers or other rate limiting species - for instance initiators - are transported by one dimensional diffusion along fibres and therefore the concentration of initiated monomers (which are capable of binding to the polymer) scales like $t^{-1 / 2}$. Thus the chain length scales as $N \sim t^{1 / 2}$. On the other hand, if there is an abundance of monomers, the polymer may grow with constant velocity, i.e. $N \sim t[45,46]$.

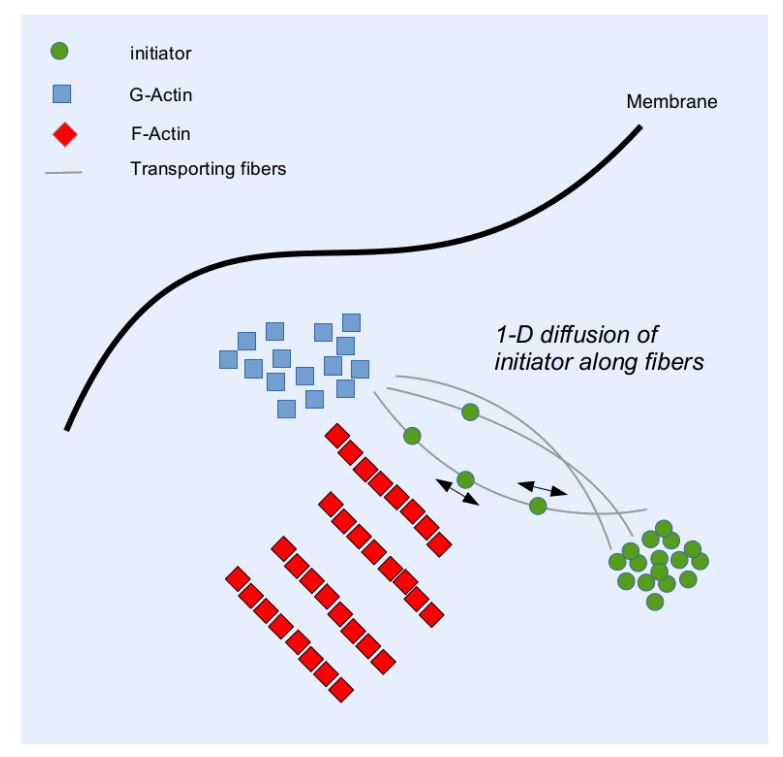

Figure 2.3: The growth law $N(t)=\gamma t^{0.5}$. In cellular environments, this situation can be imagined when the rate limiting species are diffusing in one dimension along fibers, and therefore their concentration scales as $\rho \sim t^{-0.5}$.

In Eq.(2.112) the constant $\gamma$ adds a new timescale to the problem, but we can get rid of it by an appropriate rescaling. Let us rescale the chemical space $n$, time $t$ and the 
dynamical variable $\vec{R}$ by the following factors:

$$
\begin{aligned}
\tilde{n} & :=\gamma^{\frac{1}{2 \alpha-1}} n, \\
\tilde{t} & :=\gamma^{\frac{2}{2 \alpha-1}} t, \\
\tilde{R} & :=\gamma^{\frac{1}{4 \alpha-2}} R
\end{aligned}
$$

If we substitute $\tilde{n}$ and $\tilde{t}$ in the dynamical equation for the conformation of the polymer in Eq.(2.105), we end up:

$$
\partial_{\tilde{t}} \overrightarrow{\tilde{R}}=\partial_{\tilde{n}}^{2} \overrightarrow{\tilde{R}}+\overrightarrow{\tilde{f}}(\tilde{n}, \tilde{t})
$$

where:

$$
\begin{aligned}
\langle\overrightarrow{\tilde{f}}(\tilde{n}, \tilde{t})\rangle & =0 \\
\left\langle\tilde{f}_{\alpha}(\tilde{n}, \tilde{t}) \tilde{f}_{\beta}\left(\tilde{n}^{\prime}, \tilde{t}^{\prime}\right)\right\rangle & =2 \delta_{\alpha, \beta} \delta\left(\tilde{n}-\tilde{n}^{\prime}\right) \delta\left(\tilde{t}-\tilde{t}^{\prime}\right)
\end{aligned}
$$

and:

$$
0 \leq \tilde{n} \leq \tilde{t}^{\alpha}
$$

which shows that the dimensionless parameter $\gamma$ is completely scaled out from the problem.

\subsection{Roadmap}

Now that the model for the conformational dynamics of the living polymer is set up, in the rest of this thesis we will consider various experimentally relevant scenarios and study the impact of the non-stationary growth on the conformational dynamics of living Rouse chains.

Chapter 3 is a collection of numerical and analytical tools we need to study the growing Rouse chain. We will argue, that explicit time integration schemes are not applicable in the simulation of growing polymers with decreasing polymerization rates. We will present our event-driven algorithm which turns out to be much more efficient. This algorithm is based on exactly solvability of the Rouse model between two polymerization events. Parallel to the numerical approach, we will introduce various analytical methods to solve the evolution equation of the growing chain, which -as discussed in Section 2.5.3is classified as a moving boundary problem. 
Chapter 4 is devoted to the dynamics of a free growing chain. The analytical approach allows us to distinguish different non-stationary growth regimes with qualitatively different conformational behaviour of the polymer. The analytical results will be then compared to the computer simulation.

Throughout Chapter 5 we will study a growing chain dragged from one end via a fixed force. In particular, we will demonstrate both analytically and numerically, that the distribution of the tension along the chain exhibits transition by passing from one polymerization regime to another. We will argue that this dynamical transition is a consequence of the racing duel between two dynamics: the polymerization reaction and the conformational change of the polymer.

Finally in Chapter 6, we analyse the dynamics of a growing Rouse chain under a steady shear flow. Various rheological observables will be worked out analytically. Interestingly, it will be shown that the response of the growing chain to the steady shear flow is time dependent. The results obtained from the computer simulation reveals the existence of rheological transition by going from the slow to the fast polymerisation regime. 


\section{Chapter 3}

\section{Methods}

In this chapter we introduce inportant methods, which we have used to analyse the model of conformational dynamics of a living Rouse chain. We will first explain our simulation algorithm for living chains in detail and afterwards briefly sketch the collection of analytical methods used in the following.

\subsection{Simulation algorithm}

In the previous chapter we have introduced our model, which describes the evolution of a polymer undergoing (ir)reversible polymerization. Based on this model, in this section we introduce an algorithm that simulates the conformation and the chemical reactions of such a polymerizing chain in time. As we mentioned above, the way that we described the chemical evolution of the chain is well suited for a numerical analysis using Monte Carlo techniques.

To generate a chemical path, one has to draw random waiting times $\tau_{i}$ and random chemical reaction after this waiting time $\mu_{i}$ from the waiting time distribution $p_{w}\left(\tau_{i}, \mu_{i} \mid t_{i-1}\right)$. After constructing such a path, we need to generate conformations according to probabilities $P_{\text {Rouse }}$ and $P_{\mu}$ in order to generate a full trajectory of a polymerizing chain. Here is a scetch of the algorithm that generates such trajectories: 


\section{Algorithm I}

s.1 Preparatory steps: Choose an initial conformation, simulation termination criterion, etc..

s.2 Generate a random pair $\left(\tau_{i}, \mu_{i+1}\right)$ from the waiting time distribution $p_{w}\left(\tau, \mu \mid t_{i}\right)$.

s.3 Compute the next reaction time: $t_{i+1}=t_{i}+\tau_{i}$.

s.4 Generate a conformation $\overrightarrow{\boldsymbol{R}}_{N_{i}}\left(t_{i+1}\right)$ just before the chemical reaction $\mu_{i+1}$, given the conformation $\overrightarrow{\boldsymbol{R}}_{N_{i}}\left(t_{i}^{+}\right)$.

s.5 Compute observables, e.g. the end-to-end distance, the center of mass, etc..

s.6 Generate the conformation $\overrightarrow{\boldsymbol{R}}_{N_{i+1}}\left(t_{i+1}^{+}\right)$just after chemical reaction $\mu_{i+1}$.

The Algorithm I generates trajectories of a polymerizing chain on the time intervals $\left(t_{i}, t_{i+1}\right)$ where the length of the chain is fixed, and on the reaction intervals $\left[t_{i+1}, t_{i+1}^{+}\right)$, which we assumed to be so small that the reaction $\mu_{i+1}$ happens instantaneously at $t_{i+1}$.

\subsubsection{Description of Algorithm I}

Here we describe Algorithm I in more details:

- s.1 Preparatory steps:

- Random number generator: We assume that we already have a routine that generates random numbers from standard normal distribution $\mathcal{N}(0,1)$, i.e. with a vanishing mean and unit standard deviation

- Initial conditions: The initial chain length $N_{0}$ and initial conformation $\overrightarrow{\boldsymbol{R}}_{N_{0}}$ have to be specified. We started all simulation from a double with 2 beads and the bond vector chosen from the equilibrium distribution given in Eq.(2.2).

- Termination condition: One has to specify the maximum number of chemical steps $M$. The simulation is terminated as soon as $M$ chemical reactions have been performed. In deterministic irreversible chemical reactions, $M$ is equal the maximum chain length $N_{\max }$. We terminated the simulations as soon as the chain length reached $N_{\max }=10000$ beads.

- Maximum number of trajectories: To compute observables a large number of trajectories (we chose 1000 trajectories) have to be simulated and the arithmetic mean is computed. 
- s.2 By the so called conditioning procedure[47] we split the joint probability of $(\tau, \mu)$ as $p_{w}(\tau, \mu \mid t)=p_{1}(\tau \mid t) \cdot p_{2}(\mu \mid \tau, t)$, where $p_{1}$ is the probability, that being at time $t$, the next chemical reaction (no matter what kind of reaction), happens within $[t+\tau, t+\tau+\mathrm{d} \tau)$. We can obtain this probability as:

$$
p_{1}(\tau \mid t)=p_{w}(\tau, \mu=+1 \mid t)+p_{w}(\tau, \mu=-1 \mid t)=a(t+\tau) e^{-\int_{t}^{t+\tau} a\left(t^{\prime}\right) \mathrm{d} t^{\prime}}
$$

where we defined: $a(t):=a_{p}(t)+a_{d}(t)$. The probability of the next chemical reaction $\mu$, given the waiting time for this reaction $\tau$ reads:

$$
p_{2}(\mu \mid \tau, t)=\frac{p_{w}(\tau, \mu \mid t)}{p_{1}(\tau \mid t)}=\frac{a_{\mu}}{a}
$$

Now, one can draw $\left(\tau_{i}, \mu_{i+1}\right)$ at $t_{i}$ by first drawing a waiting time from $p_{1}\left(\tau \mid t_{i}\right)$ and then drawing the type of the next chemical reaction $\mu_{i}$ from distribution $p_{2}\left(\mu \mid \tau_{i}, t_{i}\right)$.

- s.4 The seemingly simplest way to simulate the polymer chain within the nonreacting intervals $\left(t_{i}, t_{i+1}\right)$ is to use the Euler-Maruyama method[48]. Assuming that $t, t+\delta \in\left(t_{i}, t_{i+1}\right)$, for a sufficiently small $\delta$ one can convert the Langevin differential equation in Eq.(2.28) to a difference equation with a controlled error [49]. For a free chain we can write:

$$
\overrightarrow{\boldsymbol{R}}_{N_{i}}(t+\delta)=-\delta \hat{\boldsymbol{A}}_{N_{i}} \overrightarrow{\boldsymbol{R}}_{N_{i}}(t)+\sqrt{2 \delta} \overrightarrow{\boldsymbol{f}}(t)
$$

with $\hat{\boldsymbol{A}}$ as given in Eq.(2.13).

By iterating the Euler scheme in Eq.(3.3) $\tau_{i} / \delta$ times, one can obtain the conformation of the chain at $t_{i+1}$, given the conformation at $t_{i}$.

- s.6 Now that the conformation of the chain $\overrightarrow{\boldsymbol{R}}_{N_{i}}\left(t_{i+1}\right)$ just before the reaction $\mu_{i+1}$, is given, the conformation of the chain just after the reaction $\overrightarrow{\boldsymbol{R}}_{N_{i+1}}\left(t_{i+1}^{+}\right)$ can be obtained using the transition probability $P_{\mu}$ given in Eq.(2.104). The implementation of this transition is as follows:

- Polymerization: if $\mu_{i+1}=+1$, draw $\vec{b}_{n e w}$ from the Gaussian probability distribution $P_{b}\left(b_{\text {new }}\right)$ characterized by its first and second moments given in Eqs. (2.102) and (2.103) respectively. Introduce a new position at $\vec{R}_{N_{i+1}}\left(t_{i+1}^{+}\right) \leftarrow$ $\vec{R}_{N_{i}}\left(t_{i+1}\right)+\vec{b}_{\text {new }}$.

- Depolymerization: if $\mu_{i+1}=-1$, the existing bead at the tip is destroyed. In our algorithm, we accomplish this task only by updating the chain length $N_{i+1} \leftarrow N_{i}-1$. 


\subsubsection{Problem with Euler schemes}

For the deterministic power-law growth given in Eq.(2.112), the waiting time between two reactions can be calculated as:

$$
\tau_{i}=\frac{1}{\dot{N}\left(t_{i}\right)} \sim t^{1-\alpha}
$$

Recall from s.4 that in the Euler scheme $\tau_{i} / \delta$ steps are needed in order to simulate the evolution of the chain within $\left(t_{i}, t_{i}+\tau_{i}\right)$. Therefore, according to Eq.(3.4) the number of simulation steps scales as $t^{1-\alpha}$. Therefore, for $\alpha<1$ the waiting time grows without bound. This is an obvious computational problem, because for fixed $\delta$ the number of Euler steps required to simulate the chain between two reaction events increases without bound, as time passes by. The Euler scheme is therefore not applicable in this case.

In the next section, we present an alternative algorithm to the conventional Euler method, which does not encounter this problem. In fact, in our algorithm, the number of simulation steps scales like the chemical length $M$ (or equivalently with the chain length $N$ for deterministic irreversible growths), in contrast to the Euler method, where the number of simulation steps scales as $\sum_{i} \tau_{i}$.

\subsubsection{More efficient algorithm for non-stationary growth}

In this section, we introduce an alternative algorithm to the conventional Euler method, in order to simulate the chain between two reaction events $\left(t_{i}, t_{i+1}\right)$.

In our algorithm we will exploit the exact solvability of the Rouse model in order to compute the chain conformation at $t_{i+1}$, given the conformation of the chain at $t_{i}^{+}$.

Let us suppose that the conformation of the chain $\overrightarrow{\boldsymbol{R}}_{N_{i}}\left(t_{i}^{+}\right)$is given. According to Eq.(2.28), the Gaussian noise $\overrightarrow{\boldsymbol{f}}(t)$ is the only source of randomness for $\overrightarrow{\boldsymbol{R}}(t)$. In fact, Eq.(2.28) is just a system of linear Langevin equations, which can be solved by expanding $\vec{R}_{n}$ s into the eigenstates of the Rouse matrix. The corresponding modes $\vec{X}_{p}(t)$ thus define $N+1$ uncoupled Gaussian processes, which are completely characterized by their first and second moments, namely $\left\langle\vec{X}_{p}(t)\right\rangle$ and $\left\langle X_{p, \alpha}(t) X_{p, \beta}\right\rangle$ which can be calculated analytically. In the following, we present our algorithm to simulate s.4. 


\section{Algorithm II: s.4}

s.4.1 Convert positions $\overrightarrow{\boldsymbol{R}}_{N_{i}}$ into modes $\overrightarrow{\boldsymbol{X}}_{N_{i}}$ using Fast Fourier transformation [50].

s.4.2 Compute the moments $\left\langle\vec{X}_{p}\left(t_{i+1}\right)\right\rangle$ and $\left\langle X_{p, \alpha}\left(t_{i+1}\right) X_{p, \beta}\left(t_{i+1}\right)\right\rangle$ with $\alpha, \beta$ the Cartesian components and $p=0 \cdots N_{i}$.

s.4.3 Draw a random mode $\overrightarrow{\boldsymbol{X}}_{N_{i}}\left(t_{i+1}\right)$ from the Gaussian probability distribution characterized by the moments computed in the previous step.

s.4.4 Convert modes $\overrightarrow{\boldsymbol{X}}_{N_{i}}\left(t_{i+1}\right)$ into positions $\overrightarrow{\boldsymbol{R}}_{N_{i}}\left(t_{i+1}\right)$ using the inverse of the transformation utilized in s.4.1.

As we see, using the procedure above the number of simulation steps now scales as the number of the chemical events. No matter how large the time intervals between successive events, we only need the 4 steps s.4.1-s.4.4 to obtain the conformation of the chain at the end of a non-reacting interval $\left(t_{i}, t_{i+1}\right)$. For a free chain, here is the detailed description of these steps:

- s.4.1: The components of the mode $\overrightarrow{\boldsymbol{X}}$ can be expressed as the inverse transformations utilized in Eqs.(2.37), and (2.38):

$$
\begin{aligned}
\vec{X}_{p}\left(t_{i}\right) & =\sum_{n=0}^{N} \phi_{n, p} \vec{R}_{n}\left(t_{i}\right) \\
\vec{\eta}_{p}\left(t_{i}\right) & =\sum_{n=0}^{N} \phi_{n, p} \vec{f}_{n}\left(t_{i}\right)
\end{aligned}
$$

where the elements of the matrix $\phi_{n, t}$ are given in Eq.(2.35). To implement the Fast Fourier transformation the package FFTW [51].

- s.4.2: Given the Gaussian modes $\overrightarrow{\boldsymbol{X}}_{N_{i}}\left(t_{i}^{+}\right)$and using Eqs. (2.42) and (2.43) the first and second moments of the modes at $t_{i+1}$ can be calculated exactly. We can write:

$$
\begin{aligned}
\left\langle X_{0}\left(t_{i+1}\right)\right\rangle & =X_{0}\left(t_{i}\right) \\
\left\langle X_{0}^{2}\left(t_{i+1}\right)\right\rangle & =\tau_{i}
\end{aligned}
$$


and for $p>0$

$$
\begin{aligned}
\left\langle\vec{X}_{p}\left(t_{i+1}\right)\right\rangle & =\vec{X}_{p}\left(t_{i}^{+}\right) e^{-c_{p} \tau_{i}} \\
\left\langle X_{p, \alpha}\left(t_{i+1}\right) X_{p, \beta}^{2}\left(t_{i+1}\right)\right\rangle & =X_{p, \alpha}\left(t_{i}^{+}\right) \delta_{\alpha, \beta} e^{-2 c_{p} \tau\left(t_{i+1}\right)}+\frac{1}{c_{p}}\left(1-e^{-2 c_{p}\left(\tau_{i}\right)}\right) .
\end{aligned}
$$

with $c_{p}$ as given in Eq.(2.36). Note that the different Cartesian components of the modes are not correlated.

- s.4.3: If we consider the Gaussian pseudo random number $z$ drawn from $\mathcal{N}(0,1)$,

$$
X_{p}\left(t_{i+1}\right)=\left\langle X_{p}\left(t_{i+1}\right)\right\rangle+z \sqrt{\left\langle X_{p}^{2}\left(t_{i+1}\right)\right\rangle-\left\langle X_{p}\left(t_{i+1}\right)\right\rangle^{2}},
$$

has a Gaussian distribution with the moments calculated in Eqs.(3.7) - (3.10).

- s.4.4: The drawn modes are now transformed back to the configurations $\overrightarrow{\boldsymbol{R}}_{N_{i}}\left(t_{i+1}\right)$, using the Inverse Fast Fourier Transformation of step s.4.1. This is also available in package FFTW.

For a free chain, the Cartesian components are not correlated. The method can be generalized to chains in linear flows, like homogeneous shear flow, which induces correlations between different cartesian components, but still allow for an exact solution of the Langevin equations.

This procedure can be implemented to simulate flexible chains undergoing any kind of chemical reactions. The only overhead of this algorithm is the book-keeping of observables on a fixed time grid, because in calculation of averages, one has to collect observables computed at a specific time for all samples in order to calculate the arithmetic mean. The conformations, however, are computed at the stochastic reaction times. If we neglect the chemical noise and treat the evolution of $N$ as a deterministic function of time the interaction times $t_{i}$ are no longer stochastic and therefore this problem does not arise. In this case we can control simultaneously the computation time of the simulation and the number of polymerization events by specifying the maximal chain length $N_{\max }$.

\subsection{Collection of analytical tools}

The conformational dynamics of a continuous living Rouse chain has been formulated as a moving boundary value problem in Eqs.(2.105)-(2.108). We have already mentioned that problems of this type are hard to analyse. Except for special cases described in Sections 4.3 and 4.4, we did not succeed to find exact solutions. To make progress, 
we have used a bunch of combinations of transformations and perturbation methods, which are best explained in detail, when they are applied. Here, we just sketch some of the methods, which are used in several variants throughout many of the subsequent arguments.

\subsubsection{Green's function for moving boundary problems}

Formally, the solution to Eq.(2.105) can be expressed in terms of Green's function $\mathcal{G}\left(n, t \mid n^{\prime}, t^{\prime}\right)^{1}$ for a free living chain[37]:

$$
\vec{R}(n, t)=\int_{0}^{N\left(t_{0}\right)} \mathrm{d} n^{\prime} \mathcal{G}\left(n, t \mid n^{\prime}, t_{0}\right) \vec{R}\left(n^{\prime}, t_{0}\right)+\int_{0}^{t} \mathrm{~d} t^{\prime} \int_{0}^{N\left(t^{\prime}\right)} \mathrm{d} n^{\prime} \mathcal{G}\left(n, t \mid n^{\prime}, t^{\prime}\right) \vec{f}\left(n^{\prime}, t^{\prime}\right)
$$

where the Green's function satisfies:

$$
\partial_{t} \mathcal{G}\left(n, t \mid n^{\prime}, t^{\prime}\right)-\partial_{n}^{2} \mathcal{G}\left(n, t \mid n^{\prime}, t^{\prime}\right)=\delta\left(n-n^{\prime}\right) \delta\left(t-t^{\prime}\right)
$$

along with the boundary conditions of the problem. For instance, if the partial differential equation is supplemented by homogeneous boundary condition of the second kind as given in Eqs.(2.107) and (2.108), then:

$$
\begin{array}{r}
\left.\partial_{n} \mathcal{G}\left(n, t \mid n^{\prime}, t^{\prime}\right)\right|_{n=0}=0 \\
\left.\partial_{n} \mathcal{G}\left(N, t \mid n^{\prime}, t^{\prime}\right)\right|_{n=N}=0
\end{array}
$$

\subsubsection{Landau transformation}

One way to analyze the moving boundary value problem stated above is to immobilize the boundary. To this end, we perform a standard boundary fixing transformation, also known as Landau transformation[41]:

$$
\sigma:=\frac{n}{N(t)}
$$

Obviously, the range of the $\sigma$ is now fixed to:

$$
0 \leq \sigma \leq 1
$$

\footnotetext{
${ }^{1}$ In this thesis, we use $\mathcal{G}$ for the Green's function of the dynamical equation for a growing chain. The conventional letter $G$ is used for the Green's function of the Langevin equation for a fixed length chain.
} 
Therefore, substituting $n$ by $\sigma$, maps the moving boundary problem in Eqs.(2.105) (2.106) to a fixed boundary value problem. Derivatives transform as follows:

$$
\begin{aligned}
& \left.\partial_{t}\right|_{n}=\left.\partial_{t}\right|_{\sigma}+\partial_{t} \sigma \partial_{\sigma}=\left.\partial_{t}\right|_{\sigma}-\frac{\dot{N}}{N} \sigma \partial_{\sigma} \\
& \left.\partial_{n}\right|_{t}=\left.\partial_{n}\right|_{\sigma} \partial_{\sigma} \Rightarrow \partial_{n}^{2}=\frac{1}{N^{2}} \partial_{\sigma}^{2}
\end{aligned}
$$

where $\left.\partial_{x}\right|_{y}$ indicates that the function on which the derivative operator acts is defined on space $(x, y)$. Consequently Eq.(2.105) transforms to:

$$
\partial_{t} \vec{R}(\sigma, t)=\frac{1}{N^{2}(t)} \partial_{\sigma}^{2} \vec{R}(\sigma, t)+\frac{\dot{N}}{N} \sigma \partial_{\sigma} \vec{R}(\sigma, t)+\frac{\vec{\eta}(\sigma, t)}{\sqrt{N}}
$$

Obviously the immobilization of the boundaries is at the expense of complicating the evolution equation. In addition to the diffusion term $\partial_{\sigma}^{2} \vec{R}(\sigma, t)$ and the random driving force $\vec{\eta}(\sigma, t)$, the Langevin equation now contains a drift term $\partial_{\sigma} \vec{R}$. This drift arises, due to the time dependence of the Landau transformation. Another important consequence of this time-dependent transformation is the time- and space-dependent coefficients of diffusion and random terms. To eliminate the time-dependent diffusion coefficient, we introduce a new time variable by:

$$
\tau:=\int_{t_{0}}^{t} \frac{1}{N^{2}\left(t^{\prime}\right)} \mathrm{d} t^{\prime}
$$

which leads to:

$$
\partial_{\tau} \vec{R}=\partial_{\sigma}^{2} \vec{R}+(\dot{N} N) \sigma \partial_{\sigma} \vec{R}+\sqrt{N} \vec{\eta}(\sigma, \tau) .
$$

with random forces $\vec{\eta}(\sigma, \tau)$ characterized by:

$$
\begin{aligned}
\langle\vec{\eta}\rangle & =0 \\
\left\langle\eta_{\alpha}(\sigma, \tau) \eta_{\beta}(\sigma, \eta)\right\rangle & =2 \delta_{\alpha, \beta} \delta\left(\tau-\tau^{\prime}\right) \delta\left(\sigma-\sigma^{\prime}\right)
\end{aligned}
$$

The boundary condition have to be transformed, too. For instance, von Neumann boundary conditions are transformed as:

$$
\begin{aligned}
\left.\partial_{n} \vec{R}(n, t)\right|_{n=0} & =g_{0}(t) \Rightarrow & \left.\partial_{\sigma} \vec{R}\right|_{\sigma=0} & =g_{0}(t) N(t) \\
\left.\partial_{n} \vec{R}(n, t)\right|_{n=N(t)} & =g_{1}(t) \Rightarrow & \left.\partial_{\sigma} \vec{R}\right|_{\sigma=1} & =g_{1}(t) N(t)
\end{aligned}
$$

Despite the difficulties caused by the time-dependent Landau transformation, the big advantage of this transformation is that we can utilize a bunch of methods and techniques developed for problems with fixed-boundaries to solve this stochastic drift-diffusion 
equation $^{2}$. For instance, we may use perturbation methods to determine the leading behavior of the system in different time regimes. Or we may try to find the Green's function of the convection-diffusion operator $\mathcal{G}\left(\sigma, t \mid \sigma^{\prime}, t^{\prime}\right)$ or $\mathcal{G}\left(\sigma, \tau \mid \sigma^{\prime}, \tau^{\prime}\right)$ using well-known approximate methods (for special cases it can even be found exactly). To construct Green's functions, one may use mode expansions or other methods, which are particularly developed for fixed-boundary problems.

It is also possible to take advantage of numerical methods developed for Stochastic Partial Differential Equations to find the solution of this equation numerically. Such numerical methods as for example finite element methods and finite difference method are based on discretization of time and space and consequently conversion of the partial differential equation to a set of ordinary differential equations[53]. Reference [54] contains an extensive list of such methods. These numerical schemes, however, have a low order of convergence[55]. This difficulty, in terms of computational effort becomes even more severe, if the coefficients are variable, which is the case in Eqs.(3.20) and (3.22)[56].

\subsubsection{Perturbation methods}

A standard approach of theoretical analysis is a regular or singular perturbation expansion, which starts from identifying the controlling factors, (i.e. those terms which give rise to the leading order of the solution) by the method of dominant balance[57].

In this method, the terms of the dynamical equation are compared and the dominant terms in different regimes of parameters are identified. Then a perturbation expansion is set up, which starts from these terms as zeroth order. For the purpose of our discussions let us denote the partial stochastic equation (3.20) including the boundary conditions in an operator notation as in a very general form as:

$$
\mathcal{L}_{\sigma, t} \vec{R}=\vec{\Phi}(\sigma, t)
$$

which can be split into two operators as:

$$
\mathcal{L}_{\sigma, t}=\mathcal{E}_{\sigma, t}+\epsilon \mathcal{F}_{\sigma, t}
$$

For a regular perturbation problem we assume that the equation:

$$
\mathcal{E}_{\sigma, t} \vec{R}=\vec{f}(\sigma, t)
$$

is exactly solvable for a sufficiently large set of non-homogeneities $\vec{f}$. Additionally, suppose that this splitting is chosen, such that $\epsilon \mathcal{F}_{\sigma, t} \vec{g}(\sigma, t)$-in some problem-specific

\footnotetext{
${ }^{2}$ In some textbooks convection-diffusion equation[52]
} 
sense - is much smaller than $\mathcal{E}_{\sigma, t} \vec{g}(\sigma, t)$ for a sufficiently large set of functions $\vec{g}(\sigma, t)$. Let us suppose that $\vec{R}_{0}(\sigma, t)$ satisfies:

$$
\mathcal{E}_{\sigma, t} \vec{R}_{0}(\sigma, t)=\vec{\Phi}(\sigma, t)
$$

Our hope is that $\vec{R}_{0}$ is the leading order of the full solution $\vec{R}$. To test whether this splitting works, we decompose the full solution as:

$$
\vec{R}=\vec{R}_{0}+\vec{R}_{1}+o\left(\vec{R}_{1}\right)
$$

If $\vec{R}_{0}$ is the leading order, then $\vec{R}_{1}$ must be (in the problem-specific sense) much smaller than $\vec{R}_{0}$. To find $\vec{R}_{1}$, we plug Eq.(3.31) into the main equation and take advantage of the linearity of the operator $\mathcal{L}_{\sigma, t}$ :

$$
\mathcal{E}_{\sigma, t} \vec{R}_{0}+\mathcal{E}_{\sigma, t} \vec{R}_{1}+\epsilon \mathcal{F}_{\sigma, t} \vec{R}_{1}+\epsilon \mathcal{F}_{\sigma, t} \vec{R}_{0}=\vec{\Phi}
$$

This equation can now be simplified as:

$$
\mathcal{E}_{\sigma, t} \vec{R}_{1}=-\epsilon \mathcal{F}_{\sigma, t} \vec{R}_{0}
$$

where we neglect $\epsilon \mathcal{F}_{\sigma, t} \vec{R}_{1}$ compared to $\mathcal{E}_{\sigma, t} \vec{R}_{1}$. If this is correct $\vec{R}_{1}=o\left(\vec{R}_{0}\right)$, then we can conclude that the splitting is consistent and $\mathcal{E}$ is the controlling factor determining the leading order of the solution. Otherwise, one has to reconsider the splitting and try to collect those terms into the operator $\mathcal{E}_{\sigma, t}$ which yield a correct $\vec{R}_{0}$. One can go through the above-mentioned procedure to find $\vec{R}_{2}, \vec{R}_{3}, \cdots$ iteratively, and consequently approximate the solution $\vec{R}$ up to an arbitrary precision:

$$
\vec{R} \approx \vec{R}_{0}+\vec{R}_{1}+\vec{R}_{2}+\cdots
$$

This iterative strategy to find the correction of the leading order is called the perturbation method.

It may fail if the zeroth order equation (3.30) does not have a solution. An important case of this type appears, if $\epsilon$ multiplies the highest derivative of the operator $\mathcal{L}$. Then for $\epsilon=0$ the boundary conditions cannot be fulfilled in general, because the order of the unperturbed equation $(\epsilon=0)$ is lower than the order of the main equation. This is a case of a singular perturbation, for which one carefully has to distinguish between the leading order behaviour of the full solution and the solution of the unperturbed system. 


\section{Chapter 4}

\section{Dynamics of a Free Living Rouse Chain}

\subsection{Regimes of non-stationary growth}

Throughout this chapter we are mainly concerned with the conformational dynamics of a free chain growing from one end with $N(t)=\gamma t^{\alpha}$. In particular we will see in the following that there are at least three different regimes of $\alpha$ with qualitatively different behaviour of the conformational dynamics of the chain.

In a sufficiently slow polymerization, one expects that, between two polymerization events, the chain has enough time to relax and reach equilibrium state. In such situations, where the timescales of the problem are sharply separated, the utility of adiabatic approximation is justified[58]. In the adiabatic approximation, the system is supposed to be in a quasi-steady state. For a slowly growing polymer, this means that the conformation of the polymer, as the fast process in the system, between two polymerization events, can be substituted by its equilibrium conformation for a fixed length chain with length $N$. Therefore, the time evolution of the polymer conformation can be obtained by substituting the fixed length $N$ by the slow growth $N(t)$.

Now the question is, how slow the growth process should take place, in order for the quasi-steady state approximation to be justified. From Section 2.2, we already know that $\tau_{R}$, the longest relaxation time of a Rouse chain, scales as $\tau_{R} \sim N^{2}$. The waiting time between two polymerization steps, supposing the deterministic power-law growth given in Eq.(2.112) scales as $\tau_{w} \sim t^{1-\alpha}$. We require that the chain whole chain relaxes between two successive polymerization events. Therefore the relaxation time of the chain should 
be much smaller than the waiting time between two successive chemical reactions:

$$
N^{2}(t)=o\left(t^{1-\alpha}\right) \Rightarrow \alpha<\frac{1}{3}
$$

Therefore, for $\alpha<1 / 3$ the chain conformation evolves in a quasi steady state fashion. In this polymerization regime, the dynamics of growing polymers is settled: Calculate the properties of a fixed length chain in equilibrium and replace $N(t)$ by $N$ in the corresponding formulas. Beyond $\alpha=\frac{1}{3}$, however, the adiabatic approximation fails and we need another approach.

We are interested in the long-time behaviour of the system. As will be shown in the following, we will introduce a crossover time $t_{c}$ and try to determine the behavior of the polymer chain for the time limit $t \gg t_{c}$. Note that the asymptotic solution we propose, does not necessarily match to the actual behavior of the chain in the short time regime $t \ll t_{c}$.

Let us next apply the method of dominant balance to Eq.(3.20). On a crossover timescale the coefficients of drift and diffusion become comparable:

$$
\frac{1}{N^{2}\left(t_{c}\right)}=\frac{\dot{N}\left(t_{c}\right)}{N\left(t_{c}\right)}
$$

In the long time regime $t \gg t_{c}$, according to Eq.(4.2), if the growth $N(t)$ takes place slower than $t^{1 / 2}$, then the dominant term in Eq.(main) is the diffusion term. $N(t)$ is faster than $t^{1 / 2}$, this relation is reversed and the drift term is the controlling factor. $t \sim t^{1 / 2}$ specifies a special regime. Here, both diffusion and drift mechanisms are competing and the dynamics of the polymer is controlled by both of them.

\begin{tabular}{l|l|ccc}
\hline regime & $\begin{array}{l}\text { growth law } \\
N(t)\end{array}$ & $\begin{array}{c}\text { dominant terms } \\
\mathcal{E}_{\sigma, t} \vec{R}=\vec{\eta}(\sigma, t)\end{array}$ & $\begin{array}{c}\text { neglected } \\
\mathcal{F}_{\sigma, t} \vec{R}\end{array}$ \\
\hline \hline Slow: & $\alpha<1 / 2$ & $\partial_{t} \vec{R}(\sigma, t)=\frac{1}{N^{2}(t)} \partial_{\sigma}^{2} \vec{R}(\sigma, t)+\vec{\eta}(\sigma, t)$ & $(4.3)$ & $\frac{\hat{N}}{N} \sigma \partial_{\sigma} \vec{R}$ \\
Balanced: & $\alpha=1 / 2$ & $\partial_{t} \vec{R}(\sigma, t)=\frac{1}{t}\left(\frac{1}{\gamma^{2}} \partial_{\sigma}^{2} \vec{R}+\frac{1}{2} \sigma \partial_{\sigma} \vec{R}\right)+\vec{\eta}(\sigma, t)$ & $(4.4)$ & - \\
Fast: & $\alpha>1 / 2$ & $\partial_{t} \vec{R}(\sigma, t)=\frac{\dot{N}}{N} \sigma \partial_{\sigma} \vec{R}(\sigma, t)+\vec{\eta}(\sigma, t)$ & $(4.5)$ & $\frac{1}{N^{2}} \partial_{\sigma}^{2} \vec{R}$ \\
\hline
\end{tabular}

TABLE 4.1: Different polymerization regimes, according to the method of dominant balance, alongside with their corresponding asymptotic dynamical equations.

For the rest of this thesis, we refer to these polymerization regimes as slow, fast and balanced respectively. Note that in this sense, slow is not synonymous for adiabatic. Table 4.1 summarizes these regimes and their governing dynamical equations. 


\subsection{Slow polymerization}

From the method of dominant balance, we have already identified that the leading asymptotic behaviour of the exact solution of Eq.(3.20), in the slow polymerization regime, may be extracted from the solution of Eq.(4.3). We see that the order of this equation is the same as the order of the original equation. Thus the asymptotic equation given in Eq.(4.3) can satisfy the same boundary condition as the original equation. Such a perturbation problem, where the leading operator $\mathcal{E}_{\sigma, t}$ is of the same order as the original operator $\mathcal{L}_{\sigma, t}$, is referred to as regular perturbation. In such perturbation problems, one expects that the exact solution of the original problem, converges smoothly to the solution of the unperturbed problem given in Eq.(4.3), as the perturbative term $\mathcal{F}_{\sigma, t}$ gets smaller and smaller.

Consider Eq.(3.22). If we rescale the position variable as:

$$
\vec{\rho}(\sigma, \tau):=\frac{\vec{R}(\sigma, \tau)}{\sqrt{N}}
$$

this leads to:

$$
\partial_{\tau} \vec{\rho}(\sigma, \tau)=\partial_{\sigma}^{2} \vec{\rho}+\vec{\eta}(\sigma, \tau)
$$

Note that the boundary condition must also be transformed appropriately. For the free chain the boundary conditions are of von Neumann type and are homogeneous.

So finally the asymptotic dynamics of a growing chain has been mapped to the dynamics of a Rouse chain of fixed length 1 defined on $(\sigma, \tau)$. This allows us to use the results of Section 2.2. To obtain the conformation $R(n, t)$ of the growing chain we have to reverse the transformations and return from $\sigma$ to $n$, from $\tau$ to $t$ and from $\rho$ to $R$ :

$$
\vec{R}(n, t)=\sqrt{N(t)}\left\{1+2 \sum_{p} \vec{X}_{p}(t) \cos \left(\frac{k_{p} n}{N(t)}\right) e^{\left[-k_{p}^{2}(\tau(t)-\tau(0)]\right.}\right\}
$$

where $k_{p}(t):=(p \pi / N)^{2}$. In contrast to the adiabatic regime, where only the $p=0$ mode contributes to the dynamics of the polymer in Eq.(4.8) all modes are present. For long times the relaxation of these modes are not exponentially, as for a fixed-length chain, but rather stretched exponentially. Stretched exponentials are applied as models for relaxational behavior of complex systems. For instance, they are used in describing relaxation of polymers in the vicinity of a glass transition point [59-61].

In analogy to Eq.(2.47) we can introduce a time-dependent Rouse timescale $\tau_{R}(t):=$ $\frac{N^{2}(t)}{\pi^{2}}$, which scales like $t^{1-2 \alpha}$. 
At this point, let us compute some of the important observables of a slowly growing free chain. In Section 2.3 we found that the center of mass for a fixed-length chain of length 1 moves diffusively in space. Therefore, for the mean-squared displacement of the center of mass we have:

$$
\left\langle\vec{\rho}_{c o m}^{2}(\tau)\right\rangle \sim \tau .
$$

To find out how the center of mass of a slowly growing chain evolves in time, we transform $\rho_{\text {com }}$ back to $R_{\text {com }}$. Using Eq.(4.6):

$$
\left\langle\vec{R}_{\text {com }}^{2}(t)\right\rangle \sim t^{1-\alpha}
$$

Note that the center of mass of a slowly growing chain is growing sub-diffusively, in contrast to a fixed-length chain, which moves diffusively.

From the Section 2.3.2, we have seen that in the long time regime, other monomers in the chain should follow the center of mass. Therefore, for the base point at $n=0$ as well as the tip point at $n=N(t)$ we can write:

$$
\left\langle\vec{R}^{2}(0, t)\right\rangle \sim\left\langle\vec{R}^{2}(N(t), t)\right\rangle \sim t^{1-\alpha}
$$

Later we may make use of these results in Section 4.5.

Next, we calculate the autocorrelation of the end to end vector for a growing chain in the slow polymerization regime. Considering the result obtained in Eq.(2.67) for a fixed length chain asymptotically for large $\tau$ we can write:

$$
\left\langle\vec{\rho}_{e e}(\tau) \cdot \vec{\rho}_{e e}\left(\tau_{0}\right)\right\rangle \rightarrow \frac{24}{\pi^{2}} e^{-\pi^{2}\left(\tau-\tau_{0}\right)}=A e^{-\left(\frac{t}{\theta}\right)^{\beta}} .
$$

where we defined $\beta:=1-2 \alpha, \theta:=\left(\frac{\gamma^{2} \beta}{\pi^{2}}\right)^{\frac{1}{\beta}}$ and $A:=\frac{24}{\pi^{2}} e^{\left(\frac{t_{0}}{\theta}\right)^{\beta}}$. Finally we can write:

$$
\left\langle\vec{R}_{e e}(t) \cdot \vec{R}_{e e}\left(t_{0}\right)\right\rangle \rightarrow \frac{24}{\pi^{2}} t^{\alpha / 2} e^{-\left(\frac{t}{\theta}\right)^{\beta}}
$$

Thus the chain relaxes stretched exponentially, in contrast to the exponential relaxation of a fixed-length chain.

To test our asymptotic solution, we simulated such a polymerizing chain, for $\alpha=0.39$, using our numerical algorithm presented in Section 3.1. Fig.4.1 depicts this quantity as a function of time. For the sake of comparison, we also sketched the exponential decay of two fixed length chains with lengths $N=5000$ and $N=10000$ quasi monomers.

As we see, the best fit to the simulation result is the stretched exponential with variable coefficient, which we presented in Eq.(4.13). 


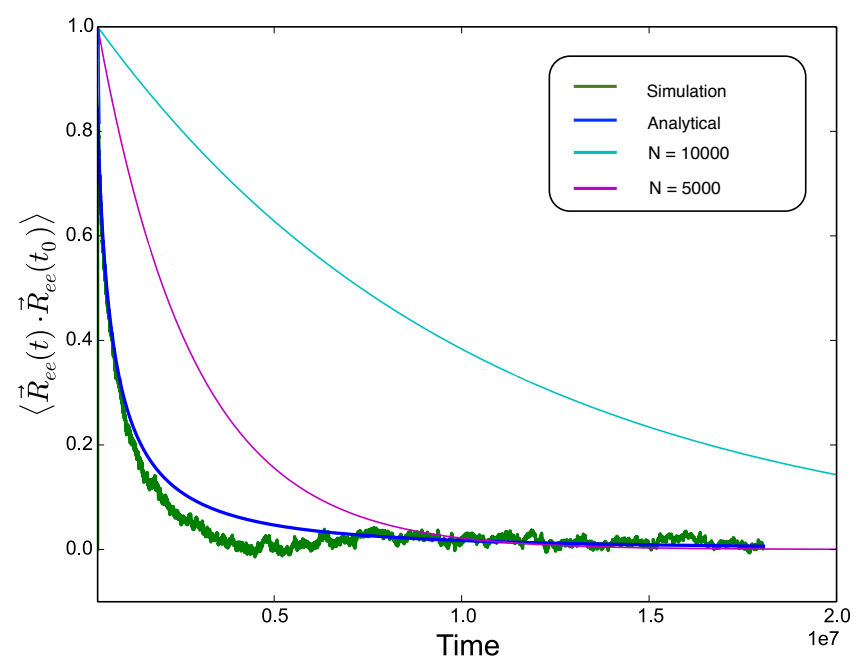

FIGURE 4.1: End to End distance correlation for a growing chain with $N(t)=t^{0.39}$ obtained from computer simulation(green). Compared to the exponential decay of fixed length chains (cyan and purple), the analytical result obtained from the method of dominant balance (blue) best matches to the simulation results.

\subsection{Balanced polymerization: exact solution for $\alpha=1 / 2$}

In this section, we study the behaviour of a free growing polymer, which is growing as $N(t)=\gamma t^{1 / 2}$. Here we may make use of the results of the Appendix B. Applying the transformation in Eq.(B.2) to Eq.(3.22) will change the homogeneous von Neumann boundary conditions into the Robin type boundary conditions as shown in Section B, which considerably complicates its solution. Note however, that homogeneous Dirichlet boundary conditions are untouched by the transformation. Therefore we prefer to work with the bond vectors $\vec{b}$ instead of $\vec{R}$. Introducing $\vec{B}$ as:

$$
\vec{b}=h(\sigma, \tau) \vec{B}(\sigma, \tau),
$$

and using Eq.(B.18) we end up:

$$
\partial_{\tau} \vec{B}=\partial_{\sigma}^{2} \vec{B}+\frac{\ddot{N} N^{3}}{4} \sigma^{2} \vec{B}+\vec{\psi}
$$

For the power-law growth with $\alpha=1 / 2$ we have $\ddot{N}=-\frac{\gamma}{4} t^{-\frac{3}{2}}$ and find that the coefficient of $\sigma^{2}$ becomes time-independent. Therefore Eq.(4.15) takes the following form:

$$
\partial_{\tau} \vec{B}=\partial_{\sigma}^{2} \vec{B}-\left(\frac{\gamma}{2}\right)^{4} \sigma^{2} \vec{B}+\vec{\psi}(\sigma, \tau)
$$

To solve this equation we construct the corresponding Green's function $\tilde{\mathcal{G}}\left(\sigma, \tau \mid \rho, \tau^{\prime}\right)$. The full solution can then be written as integrations over the Green's function (see Eq.(2.60)). 
As we see, the homogeneous part of Eq.(4.16) now can be interpreted as the imaginary time Schrödinger equation of a harmonic oscillator in a finite box with hard walls [62]. The Green's function for this equation can be written as[63]:

$$
\tilde{\mathcal{G}}\left(\sigma, \tau \mid \rho, \tau^{\prime}\right)=\sum_{p=1}^{\infty} y_{p}(\sigma) y_{p}(\rho) e^{-\lambda_{p}\left(\tau-\tau^{\prime}\right)}
$$

where $\lambda_{p}$ and $y_{p}(\sigma)$ are the eigenvalues and corresponding normalized eigenfunctions of the following boundary value Sturm-Liouville problem:

$$
\begin{aligned}
\partial_{\sigma}^{2} y(\sigma) & +\left[\lambda-\left(\frac{\gamma}{2}\right)^{4} \sigma^{2}\right] y(\sigma)=0 \\
y(0) & =0 \\
y(1) & =0
\end{aligned}
$$

The differential equation is solved by parabolic cylinder functions[64]. To solve the boundary value problem, the zeroes of solutions have to be matched at $\sigma=0$ and $\sigma=1$.

Now that we have mapped the moving boundary value problem to a standard problem of quantum mechanics, we can make use of all the well-known results from quantum textbooks.

For example, we can find the eigenvalues $\lambda_{p}$ which are all real and different. They are ordered as $\lambda_{1}<\lambda_{2}<\cdots<\lambda_{p}$, with $\lambda_{p} \rightarrow \infty$ as $n \rightarrow \infty$. Due to the fact that $\left(\frac{\gamma}{2}\right)^{4} \sigma^{2}>0$, one can conclude that $\lambda_{1}>0$ and therefore all eigenvalues are positive. Therefore, in the long time regime, the modes decay to zero exponentially in $\tau$ with time constants $\tau_{p}:=1 / \lambda_{p}$. The eigenvalues can be approximated for small $\gamma$ by standard perturbation theory as:

$$
\lambda_{p} \approx \pi^{2} p^{2}+\frac{1}{3}\left(\frac{\gamma}{2}\right)^{4}
$$

For the higher modes, one can write[63]:

$$
\begin{aligned}
\sqrt{\lambda_{p}} & =\pi p+\frac{1}{6 \pi p}\left(\frac{\gamma}{2}\right)^{4}+O\left(\frac{1}{p^{2}}\right) \\
y_{p}(\sigma) & =\sin (\pi p \sigma)-\frac{1}{6 \pi p}\left(\frac{\gamma}{2}\right)^{4}\left[\sigma\left(\sigma^{2}-1\right)\right] \cos (\pi p \sigma)+O\left(\frac{1}{p^{2}}\right)
\end{aligned}
$$

Now if we substitute $\tau=\frac{1}{\gamma^{2}} \ln (t)$ into Eq.(4.17), we find that:

$$
\tilde{\mathcal{G}}\left(\sigma, t \mid \rho, t^{\prime}\right)=\sum_{p=1}^{\infty} y_{p}(\sigma) y_{p}(\rho)\left(t \cdot t^{\prime}\right)^{-\lambda_{p} / \gamma^{2}} .
$$


This Green's function is in fact the sum over modes decaying as a power-law. The Green's function of $\vec{b}(n, t)$ reads:

$$
\mathcal{G}\left(n, t \mid n^{\prime}, t^{\prime}\right)=\frac{e^{-\frac{\gamma^{2} n^{2}}{8 t}}}{\sqrt{\gamma t^{1 / 2}}}\left[\sum_{p=1}^{\infty} y_{p}\left(\frac{n}{\gamma \sqrt{t}}\right) y_{p}\left(\frac{n^{\prime}}{\gamma \sqrt{t^{\prime}}}\right)\left(t \cdot t^{\prime}\right)^{-\lambda_{p} / \gamma^{2}}\right] \frac{\sqrt{\gamma t^{\prime 1 / 2}} e^{+\frac{\gamma^{2} n^{\prime 2}}{8 t^{\prime}}}}{\left(\gamma^{3} t^{\prime 3 / 2}\right)}
$$

Note that the factor $1 / N^{3}\left(t^{\prime}\right)$ is due to the Jacobian of the transformation $(\sigma, \tau) \rightarrow$ $(n, t)$.

\subsection{Fast polymerization and exact solution for $\alpha=1$}

In the fast polymerization regime, according to the method of dominant balance we discussed in Section 3.2.3, the drift term dominates the whole dynamics. However, the procedure presented to find the asymptotic behavior in the slow polymerization regime is not applicable here.

In contrast to the regular perturbation problem, in the fast polymerization regime, the perturbation is singular. In a singular perturbation problem, the exact solution to the original problem deviates thoroughly from the unperturbed problem. Let us take a look at Eq.(4.5). No matter how small the perturbative term is, the order of Eq.(4.5) is 1, while the order of Eq.(3.20) is 2. Therefore, there is no solution to the unperturbed problem, that satisfies the conditions at both boundaries and consequently the solution obtained from Eq.(4.5) differs in nature from the full solution of the original problem, even for $t \gg t_{c}$, where the coefficient of the perturbative term $\partial_{\sigma}^{2}$ gets smaller and smaller, compared to the drift term.

In the fast polymerization regime, nevertheless, there is one case for which the full exact solution can be found. Such a special, yet experimentally important case happens, when the growth takes place with a constant rate $\gamma$. In this case the growth-law, on average, takes the linear form $N(t)=\gamma t$. In the following, we will describe the solution of this case. This special case, as we will discuss, provides us with a better understanding of the behavior of the polymers in the fast polymerization regime.

Consider Eq.(4.15). For the case $N \sim t$, we have $\ddot{N}=0$ and therefore the equation of motion for $\vec{B}$ reduces to the simple diffusion equation in presence of a non-homogeneity $\vec{\psi}:$

$$
\partial_{\tau} \vec{B}=\partial_{\sigma}^{2} \vec{B}+\vec{\psi}
$$


The Green's function of the transformed problem thus corresponds to:

$$
\tilde{\mathcal{G}}\left(\sigma, \tau \mid \rho, \tau^{\prime}\right)=G_{D}\left(\sigma, \tau \mid \rho, \tau^{\prime}\right)=2 \sum_{p=1}^{\infty} \sin (\pi p \sigma) \sin \left(\pi p \tau^{\prime}\right) e^{-(p \pi)^{2}\left(\tau-\tau^{\prime}\right)} .
$$

We can make use of this simple transformed equation 4.24 and 4.25 to calculate observable properties of the growing chain exactly. In the following we will discuss further simplification of the Green's function in the long time regime.

In Section 2.3.2 we have given an alternative form of $G$, derived by the method of images. For Dirichlet boundary conditions, this form becomes:

$$
\tilde{\mathcal{G}}\left(\sigma, \tau \mid \rho, \tau^{\prime}\right)=\frac{1}{2 \sqrt{\pi\left(\tau-\tau^{\prime}\right)}} \sum_{p=-\infty}^{\infty}\left[e^{-\frac{(\sigma-\rho+2 p)^{2}}{4\left(\tau-\tau^{\prime}\right)}}-e^{-\frac{(\sigma+\rho+2 p)^{2}}{4\left(\tau-\tau^{\prime}\right)}}\right] .
$$

let us now take a closer look at the new time variable $\tau$, which was defined in Eq.(3.21). Remember that $\tau$ was introduced, such that $N^{2}(t) \partial_{t}=\partial_{\tau}$. Therefore:

$$
\tau:=\int_{t_{0}}^{t} \frac{1}{N\left(t^{\prime}\right)^{2}} \mathrm{~d} t^{\prime}=\frac{1}{\gamma^{2}}\left(\frac{1}{t_{0}}-\frac{1}{t}\right)
$$

which implies that tau only runs over the finite interval:

$$
0 \leq \tau \leq \tau_{\max }=\frac{1}{\gamma^{2} t_{0}}
$$

where the initial time $t_{0}$ must be larger than the crossover time $t_{c}$. In particular, if $t_{0} \gg \pi^{2} / \gamma^{2}$, we are in the short-time regime of the transformed chain, and thus can use the results of Section 2.3.2 for all $\tau$, i.e, for $t_{0} \leq t \leq \infty$.

Note that for $t \rightarrow \infty$, we have $\tau \rightarrow \tau_{\max }$. Let us choose $t_{0} \gg \frac{\pi^{2}}{\gamma^{2}}$. This yields:

$$
\tau_{\max } \ll \frac{1}{\pi^{2}},
$$

In contrast to the case of a fixed length chain this approximation is now appropriate for all times:

$$
\sum_{p=1}^{\infty} \sin (\pi p \sigma) \sin \left(\pi p \tau^{\prime}\right) e^{-(p \pi)^{2}\left(\tau-\tau^{\prime}\right)} \approx \int_{0}^{\infty} \sin (\pi x \sigma) \sin \left(\pi x \tau^{\prime}\right) e^{-(x \pi)^{2}\left(\tau-\tau^{\prime}\right)} \mathrm{d} x .
$$

Hence, the approximated Green's function becomes:

$$
\tilde{\mathcal{G}}\left(\sigma, \tau \mid \rho, \tau^{\prime}\right) \approx \frac{1}{2 \sqrt{\pi\left(\tau-\tau^{\prime}\right)}}\left[e^{-\frac{(\sigma-\rho)^{2}}{4\left(\tau-\tau^{\prime}\right)}}-e^{-\frac{(\sigma+\rho)^{2}}{4\left(\tau-\tau^{\prime}\right)}}\right]
$$


which is exactly the zero ${ }^{\text {th }}$ term in the summation of Eq.(4.26). According to Eq.(B.17) the Green's function for $\vec{b}(\sigma, \tau)$ reads:

$$
\mathcal{G}\left(\sigma, \tau \mid \rho, \tau^{\prime}\right)=\frac{e^{-\frac{\gamma N(\tau)}{4} \sigma^{2}}}{\sqrt{N(\tau)}}\left\{\frac{1}{2 \sqrt{\pi\left(\tau-\tau^{\prime}\right)}}\left[e^{-\frac{(\sigma-\rho)^{2}}{4\left(\tau-\tau^{\prime}\right)}}-e^{-\frac{(\sigma+\rho)^{2}}{4\left(\tau-\tau^{\prime}\right)}}\right]\right\}\left[\frac{e^{-\frac{\gamma N\left(\tau^{\prime}\right)}{4} \rho^{2}}}{\sqrt{N\left(\tau^{\prime}\right)}}\right]^{-1} .
$$

We use the relation

$$
e^{-\frac{(\sigma \pm \rho)^{2}}{4\left(\tau-\tau^{\prime}\right)}} e^{-\frac{\gamma N}{4} \sigma^{2}} e^{+\frac{\gamma N\left(\tau^{\prime}\right)}{4} \rho^{2}}=e^{-\frac{\gamma}{4\left(N(\tau)-N\left(\tau^{\prime}\right)\right)}\left[N(\tau) \sigma \pm N\left(\tau^{\prime}\right) \rho\right]^{2}} .
$$

in order to simplify Eq.(4.32):

$\mathcal{G}\left(\sigma, \tau \mid \rho, \tau^{\prime}\right) \approx \sqrt{\frac{N\left(\tau^{\prime}\right)}{N(\tau)}} \frac{1}{2 \sqrt{\pi\left(\tau-\tau^{\prime}\right)}}\left[e^{-\frac{\gamma}{4\left(N(\tau)-N\left(\tau^{\prime}\right)\right)}\left[N(\tau) \sigma-N\left(\tau^{\prime}\right) \rho\right]^{2}}-e^{-\frac{\gamma}{4\left(N(\tau)-N\left(\tau^{\prime}\right)\right)}\left[N(\tau) \sigma+N\left(\tau^{\prime}\right) \rho\right]^{2}}\right]$.

To obtain the Green's function of the original problem, formulated in terms of $\vec{b}(n, t)$ we have to invert the transformations $(\sigma, \tau)$. It is important to note that the transformation of the Green's function also involves the Jacobian of the transformation $(\sigma, \tau) \rightarrow(n, t)$ :

$$
\mathrm{d} \sigma \mathrm{d} \tau=\left|\begin{array}{cc}
\partial_{n} \sigma & \partial_{t} \sigma \\
\partial_{n} \tau & \partial_{t} \tau
\end{array}\right| \mathrm{d} n \mathrm{~d} t=\frac{1}{N^{3}(t)} \mathrm{d} n \mathrm{~d} t
$$

As a result, the Green's function takes the astonishingly simple form:

$$
\mathcal{G}\left(n, t \mid n^{\prime}, t^{\prime}\right)=\frac{1}{2 \sqrt{\pi\left(t-t^{\prime}\right)}}\left[e^{-\frac{\left(n-n^{\prime}\right)^{2}}{4\left(t-t^{\prime}\right)}}-e^{-\frac{\left(n+n^{\prime}\right)^{2}}{4\left(t-t^{\prime}\right)}}\right]
$$

This equation is the Green's function of a freely diffusing particle in the presence of an absorbing boundary at $n=0[65]$. It satisfies the partial differential equation given in Eq.(2.109). At the growing end $n=N(t)$, however, the Green's function does not obey the boundary conditions as discussed in Section 2.3.2. There, we found that the approximation is good in a small time regime $t \ll N^{2}$. But for the growing chain with $N(t)=\gamma t$ this condition is fulfilled in a long time regime and the Green's function at $n=N(t)$ decays as $t^{-1 / 2} e^{-\gamma^{2} t}$. Note that the crossover time where $N^{2}\left(t_{c}\right) \sim t_{c}$ is consistent with the crossover time obtained in Eq.(4.2).

For $\alpha \neq 1$ we did not find an exact solution. But the approximated Green's function (4.36) turns out to be a good approximation for all $\alpha>1 / 2$. In fact, this Green's function satisfies the dynamical equation for $\vec{b}(n, t)$ given in Eq.(2.109), and it also fullfills the boundary condition at $n=0$. At $n=N(t)$, this Green's function decays as $t^{-1 / 2} e^{-t^{2 \alpha-1}}$ and therefore satisfies the boundary condition asymptotically. This allows us to calculate observable properties also in the fast polymerization regime. 
Note that if we replace (4.36) by the corresponding von Neumann Green's function it will solve the evolution equation of $\vec{R}(n, t)$ up to a misfit at the boundary $n=N(t)$ which again rapidly decreases with time. Therefore we have found a simple and efficient way to approximately calculate properties in the fast polymerization regime both for $\vec{b}(n, t)$ and $\vec{R}(n, t)$. In the next section we will discuss dynamical properties in both the slow and fast polymerization regimes and compare our analytical findings to simulation results.

\subsection{Dynamical transitions}

\subsubsection{Segmental motion}

Comparing Eq.(4.36) with the short-time behavior of a fixed-length chain (see Eq.(2.69)), we see that individual monomers in a fast polymerizing polymer, asymptotically, should exhibit the same behavior as individual monomers in a fixed-length polymer in the short time regime $t \ll \tau_{R}$. In particular, we expect a subdiffusive motion from these monomers with an exponent of $1 / 2$. For instance:

$$
\left\langle R^{2}(0, t)\right\rangle \sim t^{1 / 2}
$$

Such a behavior is essentially distinct from that observed in the slow polymerization regime. Referring back to Eq.(4.11), we thus expect to observe a transition in the behavior of individual monomers by increasing the growth exponent from $\alpha<0.5$ to $\alpha>0.5$. Figure 4.2 illustrates the mean squared displacement of growing chains with different growth exponents in time.

For $\alpha<1 / 2$ the mean squared displacement of the base monomers grow as $t^{1-\alpha}$. For $\alpha>0.5$ they all collapse to $t^{1 / 2}$. These results are in accordance with Eqs.(4.11) (4.37).

In Figure 4.3 we investigate further the evolution of the growing chains in the slow and fast polymerization regimes by computing the mean squared displacement of the center of mass as well as the tip point in the computer simulations.

For $\alpha<0.5$ the mean squared distance of the center of mass as well as the tip point at $n=N(t)$ scales as $t^{1-\alpha}$. This behavior is already predicted in Eqs.(4.10) and (4.11), where we discussed that the polymers in the slow polymerization regime evolve in a quasi-steady state fashion. In this regime, asymptotically, the individual monomers move as a whole in space and therefore we expect that the base point, tip point and the center of mass scale as $t^{1-\alpha}$. In contrast for $\alpha>1 / 2$ the mean squared distance of the 


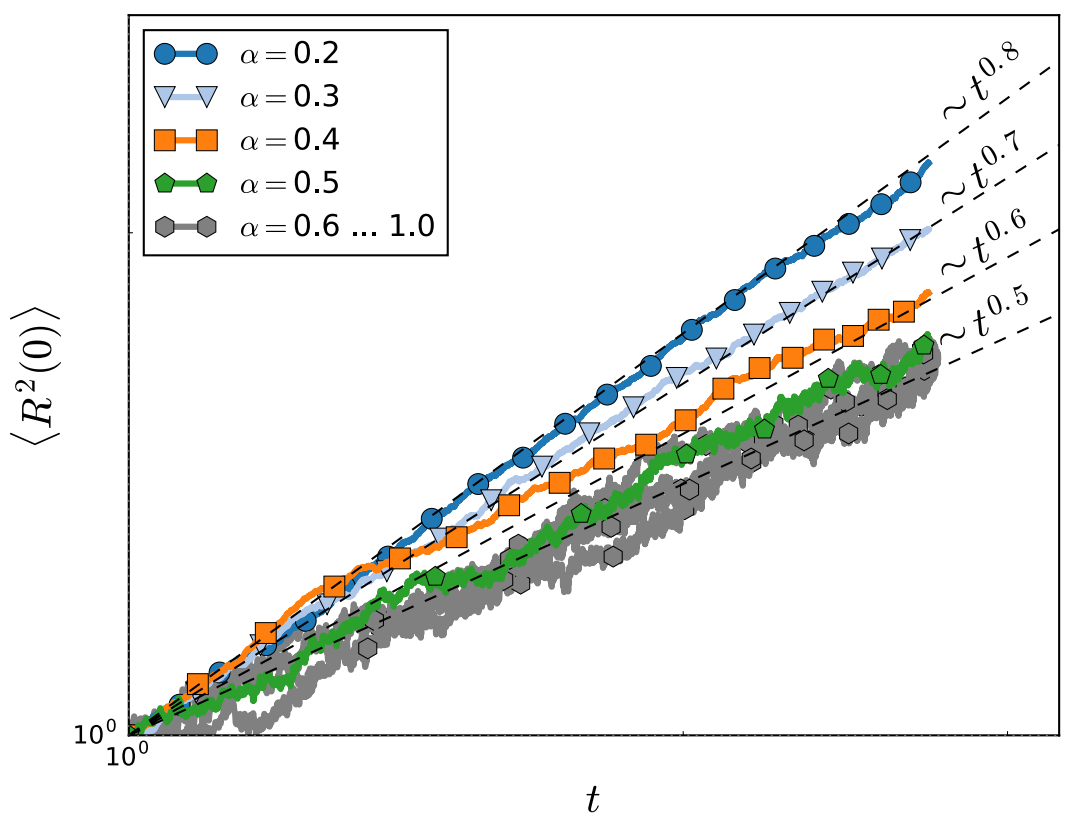

FIGURE 4.2: scaling the mean squared displacement of the base point vs. time compared to computer simulation. For the slow polymerization regime $\alpha<1 / 2$, the segments at $n=0$ evolve as Eq.(4.11). For $\alpha>1 / 2$ all curves collapse to a single curve $t^{0.5}$.
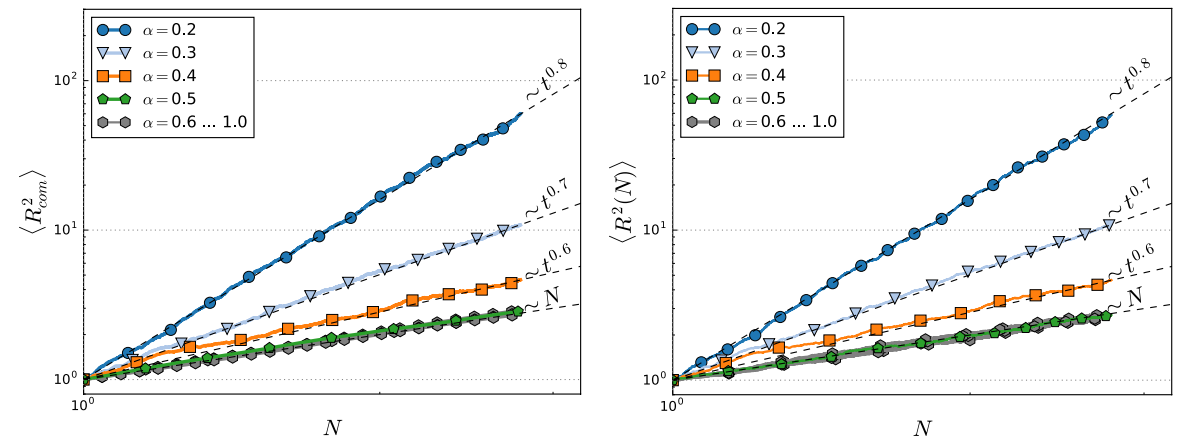

Figure 4.3: The mean squared distance of the center of mass (left) and the mean squared distance of the tip monomer at $n=N(t)$ (right) for different growth exponents as a function of the dynamic chain length $N(t)$ computed from computer simulation (see Section 3.1). For $\alpha<0.5$ the same behavior as the base point (see Figure 4.2) has been observed for the center of mass and the tip point. Increasing $\alpha$ from $1 / 2$, a different growth exponent is observed. In this regime, the mean squared distance of the tip point scales as $N$. The same behavior has been observed for the center of mass. 
center of mass and of the tip point scale as $N$. In the following, we try to explain this unexpected behavior of the tip and center of mass. Note that the Green's function of Eq.(4.36) does not satisfy the boundary condition at $n=N$ and therefore the dynamics of the tip point cannot be inferred from the approximate solution.

In particular, we will attribute this behavior to the racing duel between two stochastic terms namely the thermal noise $\vec{f}$ and the growth noise $f_{g}$ (see Section 2.5).

To understand the dynamics of the tip point in the fast polymerization regime, let us first mention, which forces are acting on this point. For a free chain there are three different sources of force acting on the tip. The first two are the same as in a fixedlength chain: the internal force due to the connectivity of the beads which we have represented in Eq.(2.6) by $\boldsymbol{F}_{\text {in }}$ and the stochastic force $\vec{f}$ as a phenomenological model for thermal collisions between the solvent molecules and the beads.

If the polymer is growing, there should be another force, which constantly moves the tip point to the position of the newly added monomers. We call this force the growth noise. At the tip point, the growth noise and the thermal noise are acting locally. On the other hand, the force exerted from any of the neighbouring beads has to travel the distance between the perturbed bead and the tip monomer. According to the Greens function of Eq.(4.36), a perturbation applied at one point on a polymer travels along the chain by diffusive mechanism. In other words, it propagates towards the tip with a velocity of $v_{\text {in }} \sim t^{-1 / 2}$. On the other hand, the tip point at $n=N(t)$ gets away from the perturbed bead with the velocity $\dot{N} \sim t^{\alpha-1}$. Hence, for $\alpha>1 / 2$ the force due to the connectivity will never arrive the tip point. In fact, in the fast polymerization regime, the tip point receives no perturbations from the existing monomers in the chain. Consequently, in analysing the motion of the tip point in the fast polymerization regime, the forces from the neighbouring beads can be neglected. We thus come to the conclusion that in the fast polymerization regime, the dynamics of the tip point is nothing other than performing Gaussian random steps at polymerization moments. This dynamics can be represented by a so-called Wiener process in the length variable $N[48]$. For such a process we have:

$$
\left\langle\vec{R}^{2}(N(t), t)\right\rangle=N(t)
$$

which explains our observation illustrated in Figure 4.3. This simple argument can also be applied to any local disturbance within the chain, as depicted in Figure 4.4. Whenever a tension is created at some bond $\vec{b}(n)$ (illustrated as event $A$ ), it spreads diffusively, and thus will reach the growing end only for $\alpha<1 / 2$. In the fast growing regime, the propagating disturbance can not reach the growing end, because the tip moves as $t^{\alpha}$ with $\alpha>1 / 2$. In other words, the tip point of a fast growing chain is causally disconnected from the existing monomers in the chain. 


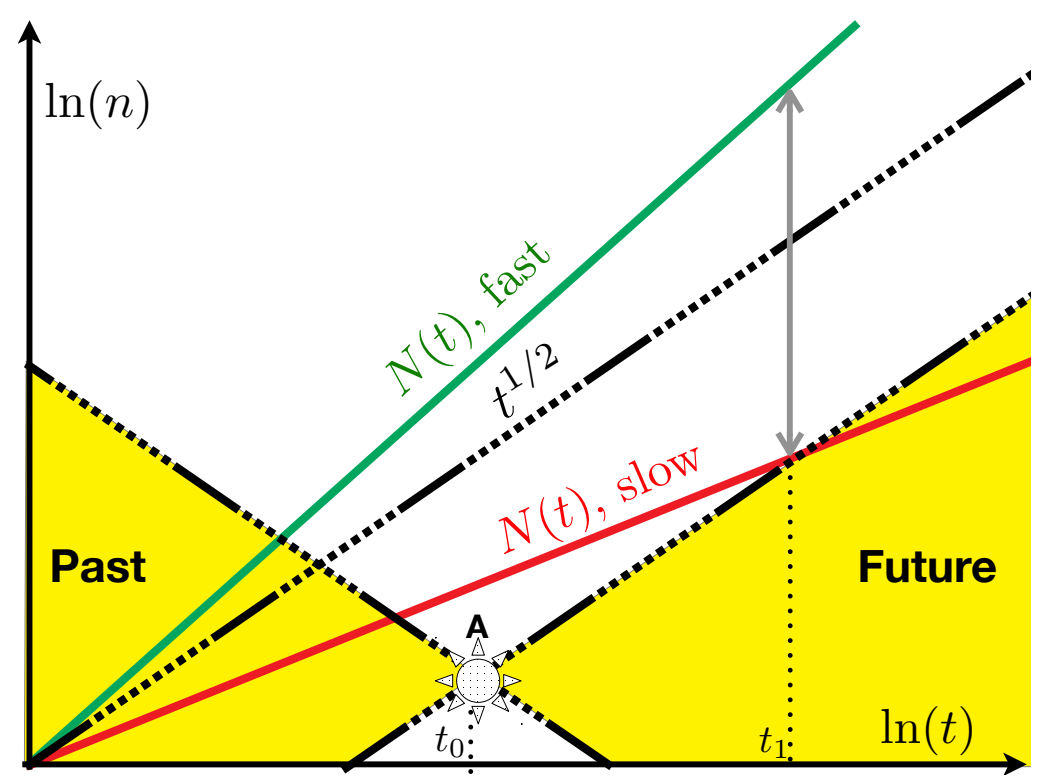

Figure 4.4: Simple physical picture to explain the dynamical behaviour of growing chains in different regimes. A change $A$ in the bond vector at time $t_{0}$ will influence other bonds by tension spreading, which proceeds as $t^{1 / 2}$ for a Rouse chain. Thus for $t>t_{0}$ (future), it can not influence the growing end of a fast growing chain, and vice versa. In fact, for a fast growing chain, the tip point is causally disconnected from the existing monomers in the chain. The picture and the reasoning resembles Minkowski diagrams.

\subsubsection{Center of mass}

Now that the behaviour of the tip point in the fast polymerization regime is settled, we can explain the dynamics of the center of mass. For $\alpha<1 / 2$, the drift term in Eq.(3.20) can be neglected compared to the diffusive term. Using this approximation, in Eq.(4.10) we derived the mean-squared displacement for the center of mass as $\left\langle\vec{R}_{\text {com }}^{2}\right\rangle \sim t^{1-\alpha}$. For $\alpha>1 / 2$, the convective term can not be neglected. Let us integrate both sides of Eq.(3.20) with respect to $\sigma$ in order to derive an equation of motion for the center of mass in the fast polymerization regime:

$$
\partial_{t} \vec{R}_{c o m}=\partial_{t} \int_{0}^{1} \vec{R}(\sigma, t) \mathrm{d} \sigma=\frac{\dot{N}}{N} \int_{0}^{1} \sigma \partial_{\sigma} \vec{R}+\frac{\vec{f}_{c o m}}{\sqrt{N}}
$$

where we plugged the boundary condition for a free chain. In addition, we defined $\vec{f}_{\text {com }}(t)$ as:

$$
\vec{f}_{\text {com }}:=\int_{0}^{1} \vec{f}(\sigma, t) \mathrm{d} \sigma
$$

which is a Gaussian point process with a vanishing average and delta correlation $\left\langle f_{\text {com }, \alpha} f_{\text {com }, \beta}\right\rangle=$ $2 \delta_{\alpha, \beta} \delta\left(t-t^{\prime}\right)$. Integrating the drift term in Eq.(4.39) by parts yields:

$$
\partial_{t} \vec{R}_{c o m}=\frac{\dot{N}}{N}\left(\vec{R}(N(t), t)-\vec{R}_{c o m}(t)\right)+\frac{\vec{f}_{c o m}}{\sqrt{N}}
$$


which can be solved as:

$$
\vec{R}_{\text {com }}(t)-\vec{R}_{\text {com }}\left(t_{0}\right)=\frac{1}{N(t)} \int_{N_{0}}^{N} \vec{R}_{t i p}\left(N^{\prime}\right) \mathrm{d} N^{\prime}+\frac{1}{N(t)} \int_{t_{0}}^{t} \sqrt{N\left(t^{\prime}\right)} \vec{f}_{c o m} \mathrm{~d} t^{\prime} .
$$

According to this equation, the dynamics of the center of mass is under the impact of two terms. The first term in the RHS is due to the thermal fluctuations of the monomers and the second term is the influence of growth in the dynamics of the center of mass. As shown in Eq.(4.42) the contribution of the thermal noise in the mean-squared displacement of the center of mass scales as $t^{1-\alpha}$. Let us calculate the mean-squared of this term to find the contribution of the growth into the mean-squared distance of the center of mass:

$$
\left\langle\left(\frac{1}{N} \int_{0}^{N} \vec{R}_{t i p}\left(N^{\prime}\right) \mathrm{d} N^{\prime}\right)^{2}\right\rangle=\frac{1}{N^{2}} \int_{N_{0}}^{N} \mathrm{~d} N^{\prime} \int_{N_{0}}^{N} \mathrm{~d} N^{\prime \prime}\left\langle\vec{R}_{t i p}\left(N^{\prime}\right) \vec{R}_{t i p}\left(N^{\prime \prime}\right)\right\rangle .
$$

In the previous section, we argued that the tip point in the fast polymerization regime should step randomly at the polymerization moments and therefore its dynamics can be considered as a Wiener process parameterized by the chain length $N$. The autocorrelation function of this process is then $\left\langle\vec{R}_{t i p}\left(N^{\prime}\right) \vec{R}_{t i p}\left(N^{\prime \prime}\right)\right\rangle=\min \left\{N^{\prime}, N^{\prime \prime}\right\}$ which results in:

$$
\left\langle\left(\frac{1}{N} \int_{0}^{N} \vec{R}_{t i p}\left(N^{\prime}\right) \mathrm{d} N^{\prime}\right)^{2}\right\rangle=\frac{1}{N^{2}}\left(\frac{N^{3}}{6}+\frac{N^{3}}{2}-\frac{N^{3}}{3}\right)=\frac{N}{3} .
$$

Therefore, compared to the thermal term, for $\alpha>1 / 2$, the dynamics of the center of mass is dominated by the tip motion. 


\section{Chapter 5}

\section{Dragged Living Rouse Chain}

Dragging single polymers such as DNA via optical tweezers has become a commonplace experiment to explore the mechanical properties of polymers[66-69]. In order to complete the picture, parallel to the experimental works, considerable amount of theoretical research has also been conducted[34,35] in order to understand the static and dynamical behaviour of dragged polymer chains. These theoretical studies are crucial to understand important phenomena, such as translocation of a polymer through a nanopore [70, 71].

Throughout this chapter, we consider the experimental setup of a growing Rouse chain dragged via a constant force from its non-growing end. Throughout this whole chapter we focus on dynamical properties of thermally averaged monomer positions $\langle\vec{R}(n, t)\rangle$ and bonds $\langle\vec{b}(n, t)\rangle$, which can be obtained from averaging Eq.(2.109) over thermal noise. To keep our notation simple, we will use $\vec{R}$ and $\vec{b}$ for these average quantities. The constant force $\vec{F}$ applied at $n=0$ is considered to be in the $x$-direction. Another notation simplification that we will use is to drop the index corresponding to the Cartesian components in the $x$ direction. Applying all these simplifications, the equation of motion in the $x$ direction reads:

$$
\partial_{t} R(n, t)=\partial_{n}^{2} R(n, t)
$$

alongside with the boundary conditions:

$$
\begin{array}{r}
\left.\partial_{n} R\right|_{n=0}=F \\
\left.\partial_{n} R\right|_{n=N}=0
\end{array}
$$

Note that in the $y$ and $z$ directions, the equations of motion are that of the free chain. 
The corresponding evolution of bond vectors (i.e. tensions) is defined by $\partial_{t} b=\partial_{n}^{2} b$ and is supplemented by the inhomogeneous Dirichlet boundary conditions:

$$
\begin{gathered}
b(0, t)=F \\
b(N, t)=0
\end{gathered}
$$

As a starting point, to get familiar with the propagation of tension along a Rouse chain, we will review the the dynamical properties of a fixed-length chain dragged at one of its ends in the next section.

\subsection{Fixed length chain}

To find the solution of a Rouse chain with a fixed length $N$, dragged at $n=0$ via a constant force $F$ (in e.g. $x$ direction), one has to solve an inhomogeneous boundary value problem. There are several well-known strategies to approach such problems. We can formulate the problem as a homogeneous boundary value problem with a singular force density $2 \delta(n) F$, concentrated at the forced end. Here, we have to consider a limiting process towards a delta function at the boundary. The correct result is recovered if we approach the delta function by a sequence of functions, which are symmetric around $n=0$.

For our discussions, we assume that the chain is initially in equilibrium and therefore $R(n, 0)=0$. Using the expansions introduced in Section 2.2 we can write:

$$
R(n, t)=F\left[\frac{t}{N}+\frac{2}{N} \sum_{p=1}^{\infty} \frac{1}{k_{p}^{2}} \cos \left(k_{p} n\right)\left(1-\mathrm{e}^{-k_{p}^{2} t}\right)\right]
$$

Let us take a look at the velocity of the forced point by differentiating this equation with respect to time:

$$
\partial_{t} R(n=0, t)=\frac{F}{N}\left(1+2 \sum_{p=1}^{\infty} \mathrm{e}^{-k_{p}^{2} t}\right) .
$$

According to this equation, there are two different time regimes, with qualitatively different behavior of the chain: For $t \ll \tau_{R}=(N / \pi)^{2}$, we can convert the summation of Eq.(5.5) into an integral over a continuous variable $p$ which leads to:

$$
\partial_{t} R(n=0, t) \approx \frac{F}{N}\left(1+2 \int_{p=1}^{\infty} \mathrm{e}^{-k_{p}^{2} t}\right) \approx \frac{1}{\sqrt{\pi t}} .
$$


Note that the velocity of the base point in the short time regime does not depend upon the chain length $N$. Consequently in the short time regime we can write:

$$
R\left(n=0, t \ll \tau_{R}\right) \rightarrow t^{1 / 2} .
$$

In the long time regime, i.e. $t \gg \tau_{R}$, the external force spreads along the whole chain and the velocity of the forced point reaches the steady state $\partial_{t} R(n=0, t) \approx \frac{F}{N}$. Therefore we expect that:

$$
R(n=0, t \rightarrow \infty) \rightarrow \frac{t}{N}
$$

The steady state tension distribution $b_{\infty}(n)$ is obtained from Eq.(5.4) as:

$$
b(n, t)=\left(1-\frac{n}{N}\right) F
$$

We can now summarize the behaviour of a dragged fixed length Rouse chain. Just a short time after applying the external force, the distortion starts to propagate along the chain by diffusion mechanism. The part of the chain close to the external force aligns to the force and takes a stretched configuration. The rest of the monomers do not feel the force and take an unperturbed configuration at rest. Therefore in this time regime, the the overall conformation of the chain possess a stem-flower like shape, with randomly coiled and stretched parts. After a sufficient time, the force distributes along the whole chain and the system reaches the steady state of Eq.(5.8). In this regime, all monomers move with the same speed and the distribution of the tension does not change in time.

\subsection{Slow polymerization}

In this section we present an analytical approach to obtain the dynamics of the bonds $b(n, t)$ in the slow polymerization regime. One can eliminate the inhomogeneous boundary condition at $n=N(t)$ by introducing a so-called shift function $b_{s h}(n, t)$. The shift function is a function that satisfies the inhomogeneous boundary condition. It must not, however, satisfy the equation of motion. For such a shift function, we choose a simple linear function in $n$ :

$$
b_{s h}(n, t)=\left(1-\frac{n}{N(t)}\right) F
$$

Now, we can decompose the solution for $\vec{b}$ into two terms $b(n, t)=b_{s h}(n, t)+b_{h}(n, t)$ : 1)The shift function which satisfies the boundary condition. 2) An unknown function $b_{h}$, which satisfies its corresponding evolution equation and is supplemented by a set of homogeneous boundary condition of the same type as the original problem (here Dirichlet boundary condition). The evolution equation for $b_{h}$ by substituting this decomposition 
into the evolution equation for $b(n, t)$. Inserting the shift function given in Eq.(5.9) yields:

$$
\partial_{t} b_{h}=\partial_{n}^{2} b_{h}-\frac{\dot{N}}{N^{2}} n F .
$$

From the boundary condition given in Eq.(5.3) we can find the boundary condition for $b_{h}$ as:

$$
\begin{gathered}
b_{h}(0, t)=b(0, t)-b_{s h}(0, t)=0 \\
b_{h}(N(t), t)=b(N, t)-b_{s h}(N, t)=0
\end{gathered}
$$

which is obviously homogeneous at both points $n=0$ and $n=N(t)$. Note that by introducing the shift function, we could transform a boundary value problem with inhomogeneous boundary condition to homogeneous boundary value problem at the expense, that the new equation of motion contains an inhomogeneity.

Now we can solve the moving boundary value problem of Eq.(5.10) using the standard techniques introduced in the previous chapter. First, we immobilize the moving boundary using the Landau transformation given in Eq.(3.16):

$$
\partial_{t} b_{h}(\sigma, t)=\frac{1}{N^{2}(t)} \partial_{\sigma}^{2} b_{h}+\frac{\dot{N}}{N} \sigma\left(\partial_{\sigma} b_{h}-F\right)
$$

Next, the time dependent coefficient of the resulting equation is eliminated using the time transformation of Eq.(3.21). Then we balance the dominant contributions and neglect small perturbations. After all these steps, the asymptotic solution of Eq.(5.10) in terms of normal modes can be obtained as:

$$
b_{h}(\sigma, \tau) \approx 2 \sum_{p=1}^{\infty}\left[X_{p}(0) e^{-k_{p}^{2} \tau}\right] \sin (\sigma \pi p)
$$

By transforming $\tau$ to $t$ and $\sigma$ to $n$ we can write:

$$
b(n, t)=\left(1-\frac{n}{N(t)}\right) F+2 \sum_{p=1}^{\infty}\left[X_{p}(0) e^{-(p \pi)^{2} \frac{t}{(1-2 \alpha) N(t)}}\right] \sin \left(\frac{n p \pi}{N(t)}\right) .
$$

and therefore in the long time regime the tension distributes along the chain as:

$$
b_{\infty}(n, t)=\left(1-\frac{n}{N(t)}\right) F
$$

In fact, according to Eq.(5.14) in the slow polymerization regime, all modes are decaying stretched exponentially in time until the tension distribution reaches the quasi steady state $b_{\infty}(n, t)$. Let us consider the bond length of a segment located at $n=\mathcal{O}(1)$. As 
time passes by, the bond length increases and for a sufficiently long time (and therefore long chain), it reaches its maximum value $b_{\infty}(n)=F$.

\subsection{Balanced polymerization}

For $\alpha=1 / 2$, we decompose $b(n, t)$ into the general solution of the homogenous problem, $b_{h}$, and a special solution of the inhomogeneous problem, $b_{b}$ as: $b=b_{h}+b_{b}$.

In Section 4.3, we presented the exact solution to $b_{h}$ as a sum over a discrete set of decaying modes. In this section we will find the particular solution $b_{b}$ that satisfies

$$
\partial_{\tau} b_{b}=\frac{1}{\gamma^{2}} \partial_{\sigma}^{2} b_{b}+\frac{1}{2} \sigma \partial_{\sigma} b_{b}
$$

In particular, we look for a stationary solution of this equation, which along side the solution given in Section 4.3 gives the full solution for $b(n, t)$. Note that the stationary solution $b(\sigma)$ still depends upon time because $\sigma=n / N(t)$. The stationary solution has to obey the ordinary differential equation:

$$
\frac{1}{\gamma^{2}} \partial_{\sigma}^{2} b_{b}=-\frac{1}{2} \sigma \partial_{\sigma} b_{b}
$$

which is easily solved by introducing $S:=\partial_{\sigma} b_{b}$ and substitute it into Eq.(5.16)

$$
\partial_{\sigma} \ln (S)=-\frac{\gamma^{2}}{2} \sigma
$$

The first order ordinary differential equation for $S$ has the solution $S=S_{0} \exp \left(-\frac{\gamma^{2}}{4} \sigma^{2}\right)$ which leads to

$$
b=S_{0} \operatorname{erf}\left(\frac{\gamma}{2} \sigma\right)+S_{1}
$$

where we have used the standard definition of the error function $\operatorname{erf}(x):=\frac{2}{\sqrt{\pi}} \int_{0}^{x} e^{-y^{2}} \mathrm{~d} y$. The constants $S_{0}$ and $S_{1}$ are to be determined from the boundary conditions given in Eq.(5.3):

$$
\begin{aligned}
& b_{b}(\sigma=0, t \rightarrow \infty)=F \\
& b_{b}(\sigma=1, t \rightarrow \infty)=0 .
\end{aligned}
$$

Hence:

$$
\begin{aligned}
& S_{1}=F \\
& S_{0}=-\frac{F}{\operatorname{erf}\left(\frac{\gamma}{2}\right)} .
\end{aligned}
$$


Finally $b_{b}$ can be written as:

$$
b_{b}(\sigma)=\left(1-\frac{\operatorname{erf}(\gamma \sigma / 2)}{\operatorname{erf}(\gamma / 2)}\right) F
$$

If we calculate the second derivative of this function as $\partial_{\sigma}^{2} b_{b}=\frac{2 F \gamma \sigma e^{-(\gamma \sigma / 2)^{2}}}{\sqrt{\pi}}$ we find that in the interval $\sigma \in[0,1]$, the function is concave up. Compared to the linear solution of Eq.(5.14), in the balanced polymerization regime the tension is more piled up at the forced end (see Figure 5.1). Now we can calculate the full solution in the long time regime by considering both transient solution $b_{h}$ and the stationary solution $b_{b}$. In the long time, when all the modes of $b_{h}$ are relaxed, the ground state for the tension reads:

$$
b_{\infty}(n)=\left(1-\frac{\operatorname{erf}\left(\frac{n}{2 \sqrt{t}}\right)}{\operatorname{erf}(\gamma / 2)}\right) F .
$$

As in the slow polymerization regime, the bonds at a fixed chemical position on the chain $n=\mathcal{O}(1)$ elongates in time and for the asymptotically long time, which equivalently means a sufficiently long chain the bond length is $b_{\infty}\left(n^{*}\right) \rightarrow F$ for monomers with fixed chemical position. Again hear the boundary condition at $n=N(t)$ is satisfied.

\subsection{Fast polymerization}

In this section, we try to find $b(n, t)$ in the fast polymerization regime. To find such a solution, we combine the variables $\sigma$ and $t$ as $z:=t \sigma^{\lambda}$ and attempt to find the power $\lambda$ in such a way that the partial differential equation consists only of derivatives with respect to $z$ (all derivatives with respect to $\sigma$ and $t$ would be eliminated). As a consequence, the evolution equation for $b(n, t)$ is transformed to an ordinary differential equation which can be solved analytically. Let us first introduce a new dynamical variable $g(z)$ as:

$$
g\left(z=t \sigma^{\lambda}\right):=b(\sigma, t)
$$

The derivatives with respect to $\sigma$ and $t$ can be written in terms of derivatives of $g$ with respect to $z$. These derivatives are $\partial_{t} b=\sigma^{\lambda} g^{\prime}, \partial_{\sigma} b=t \lambda \sigma^{\lambda-1} g^{\prime}$ and $\partial_{\sigma}^{2} b=t \lambda(\lambda-$ 1) $\sigma^{\lambda-2} g^{\prime}+\left(t \lambda \sigma^{\lambda-1}\right)^{2} g^{\prime \prime}$. Now we can substitute these relation into the equation for $b(n, t)$ to obtain:

$$
\gamma^{2} g^{\prime}-\gamma^{2} \alpha \lambda g^{\prime}-\lambda(\lambda-1) g^{\prime} t^{1-2 \alpha} \sigma^{-2}-t^{2-2 \alpha} \lambda^{2} \sigma^{\lambda-2} g^{\prime \prime}=0
$$

The free parameter $\lambda$ is to be determined, such that all terms depending upon $\sigma$ or $t$ convert to terms only dependent on $z$. This is achieved by choosing $\lambda=\frac{2}{2 \alpha-1}$. The 
resulting equation reads:

$$
\left(\lambda^{2}-\lambda^{2} a-c z^{1-2 \alpha}\right) g^{\prime}=d z^{2-2 \alpha} g^{\prime \prime}
$$

where we introduced $a:=\frac{2 \alpha}{2 \alpha-1}, c:=\left(\frac{2}{2 \alpha-1}\right)\left(\frac{3-2 \alpha}{2 \alpha-1}\right)$ and $d:=\left(\frac{2}{2 \alpha-1}\right)^{2}$. This equation can be solved as:

$$
g(z)=K_{1} \times \frac{4}{\gamma(2 \alpha-1)} \frac{\sqrt{\pi}}{2} \operatorname{Erf}(z)+K_{2} .
$$

To find the constants $K_{1}$ and $K_{2}$, let us rewrite $z$ in terms of $\sigma$ and $t$ :

$$
b(\sigma, t)=K_{1} \operatorname{erf}\left[\frac{\gamma}{2}\left(t^{\frac{2 \alpha-1}{2}} \sigma\right)\right]+K_{2} .
$$

where erf is the standard error function. This solution needs to satisfy the boundary condition given in Eq.(5.3). Applying the condition at $\sigma=0$, we can obtain $K_{2}$ as:

$$
K_{2}=F
$$

The boundary at $\sigma=1$ can not be fulfilled exactly. Nevertheless, by choosing $K_{1}=-F$, the boundary condition at this point will be satisfied asymptotically:

$$
b(\sigma=1, t) \sim \frac{\exp \left[-\left(t^{\frac{2 \alpha-1}{2}}\right)^{2}\right]}{\left(t^{\frac{2 \alpha-1}{2}}\right)}
$$

Thus, the similarity solution for the fast polymerization regime can be obtained as:

$$
b(\sigma, t) \approx F \operatorname{erfc}\left[\frac{\gamma}{2}\left(t^{\frac{2 \alpha-1}{2}} \sigma\right)\right]
$$

where erfc is the standard complementary error function. In contrast to the slow and balanced regimes, is no more stationary. In the next section we will discuss the consequences of this non-stationary tension distribution on the conformational dynamics of fast growing chains.

Transforming the variable $\sigma$ to $n$, Eq.(5.29) takes the form:

$$
b(n, t)=\operatorname{erfc}\left(\frac{n}{2 \sqrt{t}}\right) F
$$

This result resembles the solution for a semi infinite chain $n \in[0, \infty)$. Recall from Figure 4.4 , that any perturbation applied on the chain can only be received by the monomers with $n=\mathcal{O}\left(t^{1 / 2}\right)$ at time $t$. In fact, the forced point at $n=0$ and the tip point are causally disconnected which resembles the case, where the tip point is located in $\infty$. 


\subsection{Summary of the analytical results}

In the previous section, we found that the tension distributes differently along dragged chains, which are growing in different polymerization regimes. Now we can calculate various observables in different growth regimes. Table 5.1 summarizes these observables calculated for different growth regimes.

\begin{tabular}{l|c|c|c} 
& $\alpha<0.5$ & $\alpha=0.5$ & $\alpha>0.5$ \\
\hline \hline$b(n, t)$ & $\left(1-\frac{n}{N}\right) F$ & $F\left[1-\frac{\operatorname{erf}\left(\frac{\gamma n}{2 N(t)}\right)}{\operatorname{erf}\left(\frac{\gamma}{2}\right)}\right]$ & $F \operatorname{erf}\left(\frac{n}{2 \sqrt{t}}\right)$ \\
& & & \\
\hline$R_{\text {tip }}-R_{\text {com }}$ & $t^{\alpha}$ & $t^{1 / 2}$ & $t^{1-\alpha}$ \\
\hline$R_{\text {com }}(t)$ & $t^{1-\alpha}$ & $t^{\alpha}$ & $t^{1-\alpha}$ \\
\hline$R_{e e}$ & $t^{\alpha}$ & $t^{1 / 2}$ & $t^{1 / 2}$ \\
\hline$R_{0}(t)$ & $t^{1-\alpha}$ & $t^{1 / 2}$ & $t^{1 / 2}$ \\
\hline$R_{\text {tip }}(t)$ & $t^{1-\alpha}$ & $t^{1 / 2}$ & const
\end{tabular}

TABLE 5.1: Variouse dynamical properties calculated for a dragged chain, which is growing as $N(t)=\gamma t^{\alpha}$.

In the table above As we see from this table, the conformational dynamics of dragged growing chains are qualitatively different in different growth regimes. This transition will be discussed in detail in the next section.

\subsection{Dynamical transitions}

In this section we present the results obtained from computer simulation and compare them to our analytical calculations summarized in Table 5.1.

For a unit dragging force $\vec{F}=(1,0,0)$, Figure (5.1) shows $\vec{b}(\sigma, t)$ vs. $\sigma$ for $N=1000$ and $N=10000$ quasi-monomers and for different values of $\alpha$. The lines represent the analytical solutions obtained in Eqs.(5.14), (5.22) and (5.29).

For $\alpha<1 / 2$ the tension reaches the linear limit distribution $1-\sigma$ as expected. In the balanced polymerization regime $\alpha=0.5$, the distribution of the tension in the short and long times coincide and therefore it seems to be independent of time. In contrast to the slow polymerization regime, tension is distributed non-linearly along the chain. This result is in accordance with the steady state calculation of Eq.(5.22). For $\alpha>1 / 2$ the tension piles up more and more near the dragged end, while an increasing fraction of the chain remains force free. 


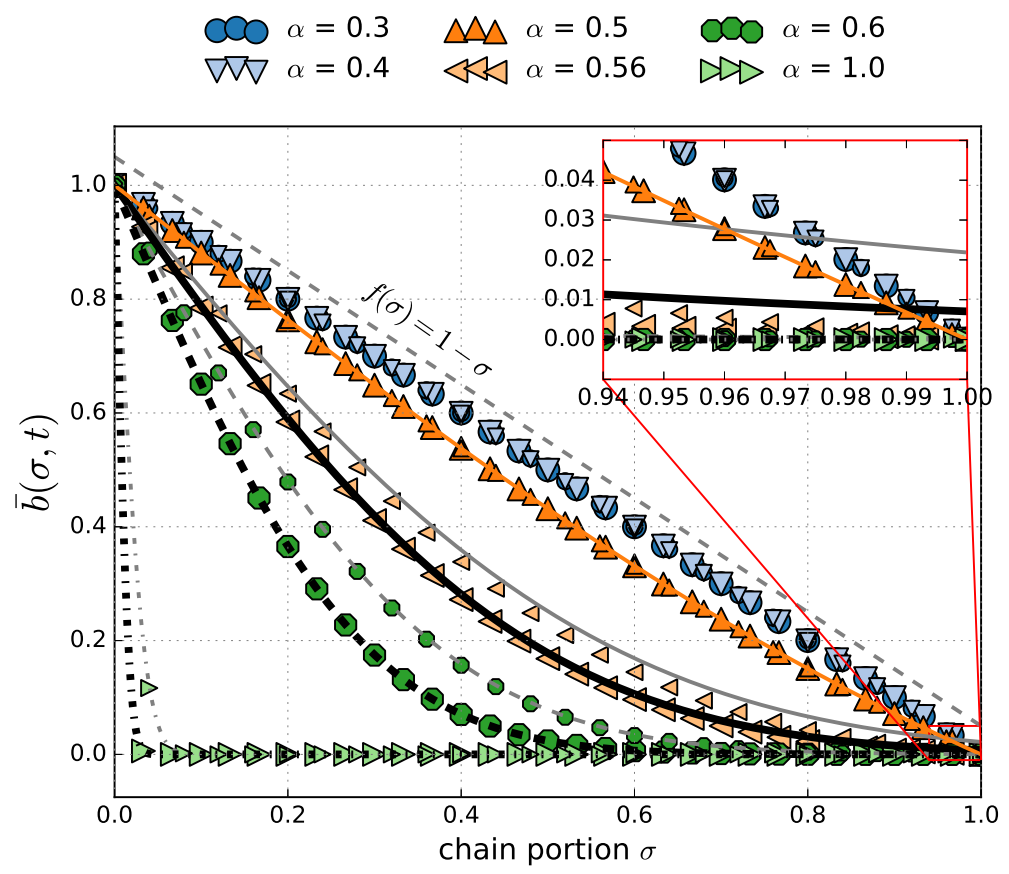

Figure 5.1: Averaged tension distribution along growing chains dragged via a unit force $\vec{F}=(1,0,0)$ as the chain lengths reach $N=1000$ (small markers) and $N=10000$ (large markers) monomers. The growth is modeled as $N(t)=1.5 t^{\alpha}$ : In the slow polymerization regime $(\alpha<0.5)$ the chain has been relaxed to a quasi-steady state conformation $\bar{b}(\sigma, t) \rightarrow(1-\sigma)$ even in the early stage $(N=1000)$. For $\alpha=0.5$, the tension distribution is time-independent but no more linear. The analytic solution (solid line) matches perfectly to the simulation result. In the fast polymerization regime $\alpha>0.5$, the force starts to pile up near the forced point. The piling up gets more pronounced as time proceeds. The grey $(N=1000)$ and black $(N=10000)$ lines show the similarity solution of Eq.(5.29). Inset: $\times 7$ magnification. For $\alpha=0.56$ the similarity solution does not satisfy the boundary condition in the short time regime. In the later time the similarity solution at $\sigma=1$ approaches to the boundary condition of the problem. Consequently the global matching is improved

As we see, by passing from the slow polymerization regime to the fast polymerization regime, the system exhibits a phase transition. To understand the physical origin of this transition, suppose that the dragging starts at $t=0$, so that the initial tension is localized at $n=0$. In Figure 4.4 we discussed that the causal connection between different compartments of the chain is affected by growth. For a dragged chain, one has to consider the causal connection between the perturbed point namely the base point and the tip point. The drag spreads diffusively along the polymer and within a time $t>0$, it will build up tensions in bonds with $n \sim O(t 1 / 2)$. If $\alpha<1 / 2$, tension propagates faster than polymer growth and will reach the growing end even for longest times. However, if $\alpha>1 / 2$ polymer growth is faster than the propagation of tension and the fraction of stretched bonds decreases with time. In other words, the transition is the result of a racing duel between the tension and the polymer growth. 


\subsubsection{Tension threshold}

To have a better understanding about the transport mechanism of tension along the chain, we tracked a certain tension on each of the chains and observed the position of this tension in time. Figure (5.2) illustrates the position $\sigma^{*}$, where the segments are under a tension equal to $0.2 \vec{F}$ for various chains growing with different exponents. For chains with $\alpha \leq 0.5$, after a sufficient time, $\sigma^{*}$ approaches a time independent limit, which is in accordance with the quasi-steady state solution of Eq.(5.14). In particular for $\alpha<0.5$ we expect (Figure 5.2):

$$
0.2 F=\left(1-\sigma^{*}\right) F \Rightarrow \sigma^{*}=0.8
$$

Increasing $\alpha$ above $0.5, \sigma^{*}$ decreases with time meaning that tension is accumulating to the forced end of the chain.

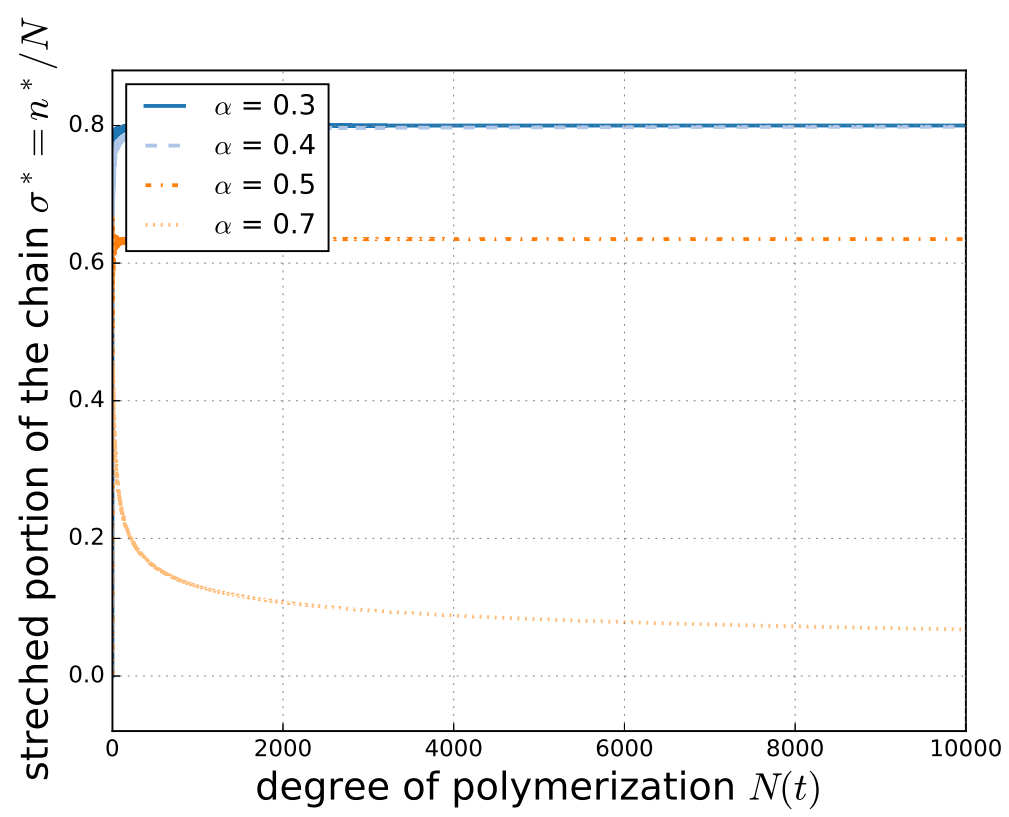

Figure 5.2: The chemical position (normalized by the chain length $N(t)$ ), where the segments are under a tension equal to $0.2 F$ for various chains growing with different growth exponents $\alpha$. For chains growing with exponents $\alpha<0.5$, the tension distribution reaches a quasi-steady state and thus the chemical position of this tension saturates to a constant value $\sigma *=0.8 F$ (see Eq.(5.31)). For the intermediate regime $\alpha=0.5$, the distribution of the tension is a non-linear quasi-steady state. Increasing $\alpha$ above $0.5, \sigma *$ decreases with time. This means that the force free portion of the chain grows in time and the tension is accumulating at the forced end of the chain. 


\subsubsection{End-to-end distance}

Figure 5.3 depicts the end to end distance normalized by the polymer length, which is computed as polymers reach certain lengths $(N=10000,5000,1000,500)$. To illustrate the transition, we sketched this quantity with respect to the growth power $\alpha$. Below $\alpha=0.5$, as can be read from Table 5.1, $R(N, t)-R(0, t)$ scales like $N(t)$ and therefore the graph approaches a fixed value. This behavior is analogous to the fixed length chain, where the average end to end distance can be computed as:

$$
R(N, t)-R(0, t)=\frac{F N}{2}
$$

For chains growing faster than $t^{0.5}$, only a portion of the chain, which scales as $t^{0.5}$ feels the force and the majority of the chain is a random coil with a vanishing averaged end to end distance. Hence the length $N(t)$ grows faster than the averaged end to end distance and the graph drops to zero. Note that for longer chains the transition at $\alpha=0.5$ is more abrupt.

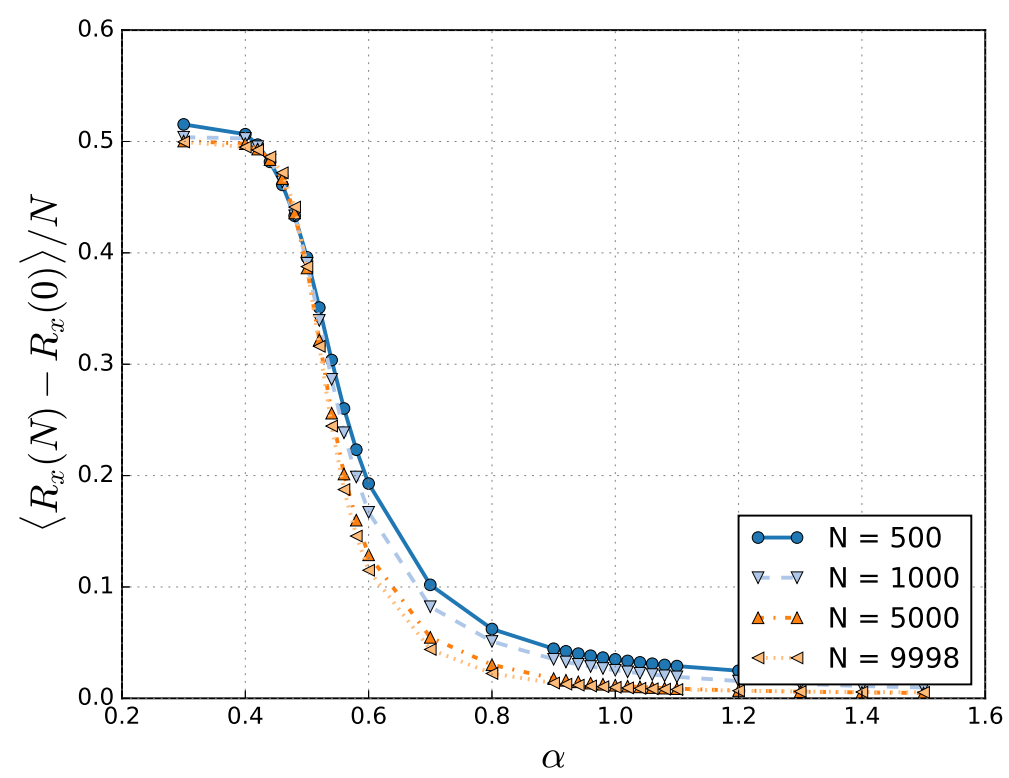

FIGURE 5.3: End-to-end distance along the direction of external force, normalized by the chain length $N$. For $\alpha<0.5$, the end to end distance scales as $t^{\alpha}$. Beyond $\alpha=0.5$, it scales as $t^{1 / 2}$. Hence the normalized end to end distance decreases sharply to zero. See Table 5.1. 


\subsubsection{Base and tip in the center of mass reference frame}

The structural transition discussed above leads to a transition in the dynamics of monomers in the reference frame of the center of mass. We observed that the position of the base and tip points in the reference frame origined at the center of mass, obeys a power-law in the long time regime. Figures (5.4a) and (5.4b) depicts the fitted exponent of these power-laws as a function of $\alpha$. For $\alpha<0.5$, both base and tip monomers move as $\sim t^{\alpha}$. Beyond $\alpha=0.5$, the behaviour of the tip point switches to a power law with an exponent of $t^{1-\alpha}$, while the exponent of the base point approaches a plateau $\alpha=0.5$.

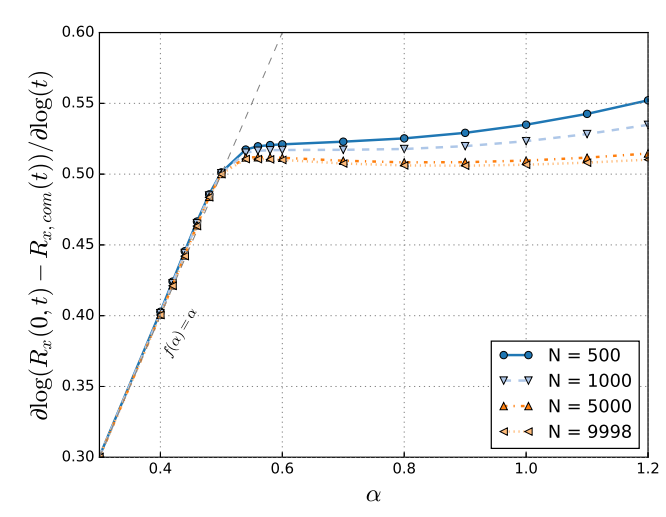

(A)

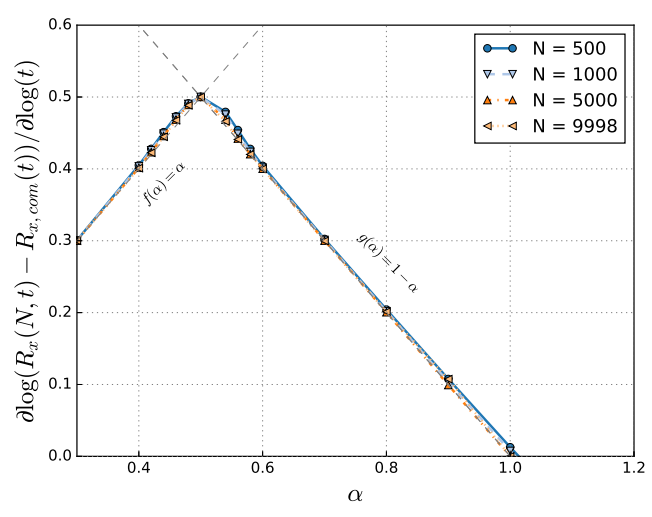

(B)

Figure 5.4: The exponents of the power-laws corresponding to the position of the base and tip point in the reference frame origined at the position of the center of mass as a function of $\alpha$. These values have been collected at different growth stages $(N=500,1000, \cdots)$. For both observables, around $\alpha=0.5$, one can see a sharp change in the exponents of the power-laws.

\subsubsection{The shape of the chain}

Figure 5.5 depicts a snapshot of a growing chain, with the growth exponent $\alpha=0.7$, as it reached $N=10000$ beads. As we see, the polymer in the fast polymerization regime possess a stem-flower like shape. The stretched part close to the forced point takes a stretched conformation, while the majority of the polymer does not feel the force and consequently is randomly coiled.

To analyse the structure of the chain shown in Figure 5.6, we measured the mean squared distance between each bead located at $n$ and the tip point at $N$ and sketched it with respect to the chemical distance between these two points namely $n_{s}:=N-n$. The data are collected as the chains reach the length $N=10000$ monomers. The random coil conformation is characterized by random walk in the chemical space and therefore 


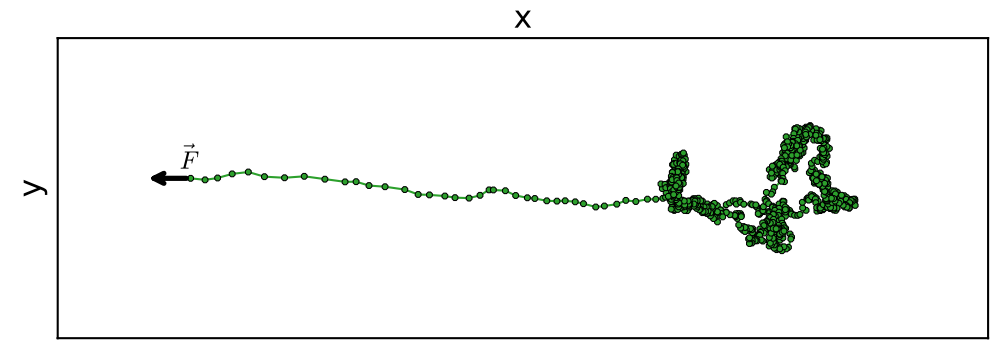

FiguRE 5.5: A snapshot of the system with $\alpha=0.7$ as the chain reaches $N=10000$ monomers. The chain conformation is a combination of random coil and streched configurations.

the mean squared distance should scale like the chemical distance. Considering Figure 5.6 , the growing end of all chains are random coils with $(R(N)-R(n))^{2} \sim n_{s}$. For the slowly growing chains, we can observe a stretched conformation with $R(N)-R(n) \sim n_{s}^{4}$ for $n_{s}>500$ which is the major portion of the chain. Beyond $\alpha=1 / 2$ no stretched conformation has been identified.

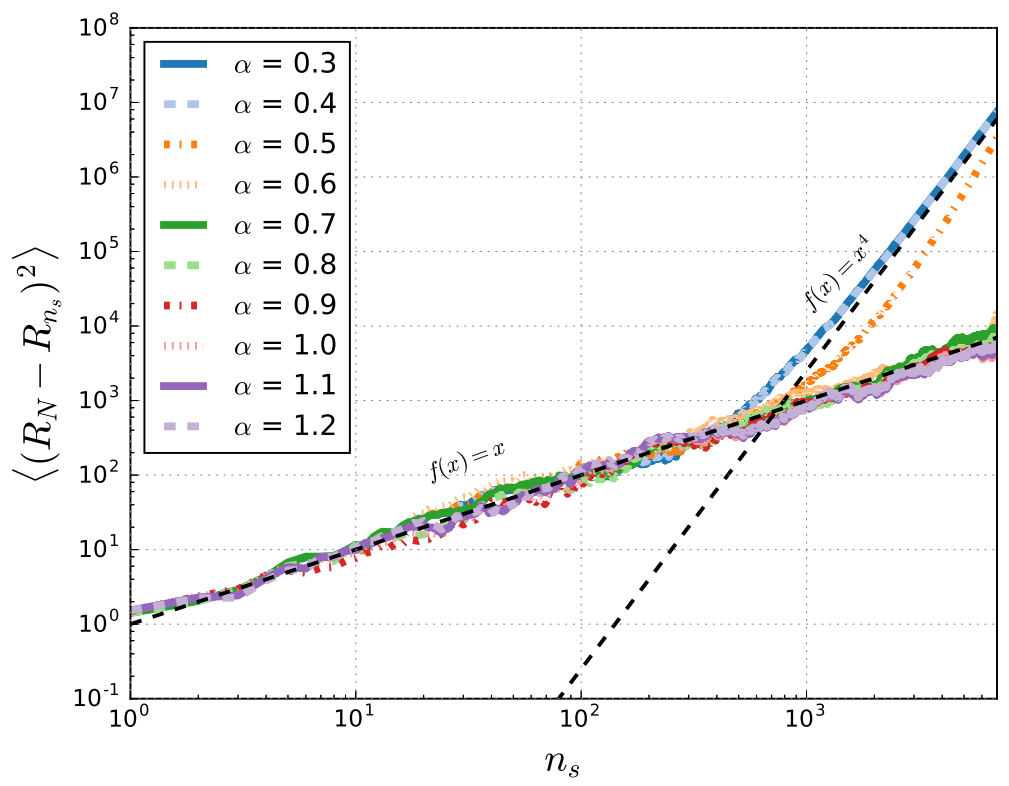

Figure 5.6: Squared distance of monomer at chain position $s=N-n_{s}$ from the tip position $R(N, t)$ vs $n_{s}$ computed from snapshots captured as the chain reaches $N=10000$ monomers. For $\alpha>1 / 2$ the influence of the dragging force is restricted to a very thin boundary layer. For $\alpha<1 / 2$ the coiled part of the chain shrinks to a boundary layer (of a few hundred monomers) near the tip. For $\alpha=1 / 2$, the stretched part of the chain does not follow a simple power-law scaling.

\subsubsection{Coupling between center of mass and tip}

As mentioned in Section 4.5 in growing chains, the dynamics of the center of mass is closely connected to the internal dynamics of the chain. We can derive the evolution 
equation for $R_{\text {com }}$ as:

$$
\partial_{t} R_{\text {com }}=\frac{\dot{N}}{N}\left(R_{\text {tip }}-R_{\text {com }}\right)-\frac{F}{N}
$$

One can multiply both sides of Eq.(5.33) by $N(t)$ and rearrange the relation above to obtain:

$$
\partial_{t}\left[N(t) \cdot R_{\text {com }}\right]=\dot{N} \cdot R_{\text {tip }}-F
$$

Note that if $\dot{N}=0$, i.e. if the growth velocity is zero, the correlation between $R_{\text {com }}$ and $R_{t i p}$ vanishes and consequently one recovers the equation of motion for the center of mass for a fixed length chain.

Rearranging the terms we obtain:

$$
\partial_{t} R_{t i p}=\frac{\partial_{t}\left[N\left(R_{t i p}-R_{c o m}\right)\right]}{N}-\frac{F}{N}
$$

Now we can substitute $\left\langle R_{\text {com }}-R_{\text {tip }}\right\rangle$ from Table 5.1 and calculate the dynamics of $\vec{R}(N(t), t)$ for the whole range of $\alpha$. For $\alpha<0.5, R_{\text {com }}-R_{t i p} \sim t^{\alpha}$. Therefore in Eq. (5.35) the velocity of the tip is mostly dominated by the term $t^{-\alpha}$ (second term in the RHS.). Hence the absolute position of the tip point scales as $R(N(t), t) \sim t^{1-\alpha}$. Beyond $\alpha=0.5$, if we also consider the prefactors $\left\langle R_{\text {com }}-R_{t i p}\right\rangle$, we obtain:

$$
\left\langle R_{\text {com }}-R_{t i p}\right\rangle=\frac{F t}{N}
$$

and substituting into Eq.(5.35) yields $R(N(t), t) \rightarrow$ const. As we see, the tip point of the polymer undergoes a non-trivial transition by passing from slow polymerization regime $\alpha<0.5$ to the fast polymerization regime $\alpha>0.5$. In fact, for $\alpha>0.5$, we expect from the discussion above that the tip performs an unbiased random walk. The numerical results confirm such an arrest of the tip point for $\alpha>0.5$ (see Figure 5.7).

Now we can use the results above to analyze the dynamics of the center of mass. For $\alpha<0.5$, substituting the value of $\left\langle R_{\text {tip }}-R_{\text {com }}\right\rangle$ from Table 5.1 into Eq.(5.33) we can obtain:

$$
\left\langle R_{\text {com }}\right\rangle \rightarrow t^{1-\alpha}
$$

Going beyond $\alpha=0.5$, the tip gets stuck at $C$ and therefore Eq.(5.34) yields:

$$
R_{\text {com }}(t) \rightarrow C+\frac{F t}{N(t)}
$$

Considering Eq.(5.38), we can see a clear dynamical transition in the motion of the center of mass at $\alpha=1$. For $\alpha<1$ the dynamics is dominated by the power-law $R_{\text {com }} \sim t^{1-\alpha}$. 


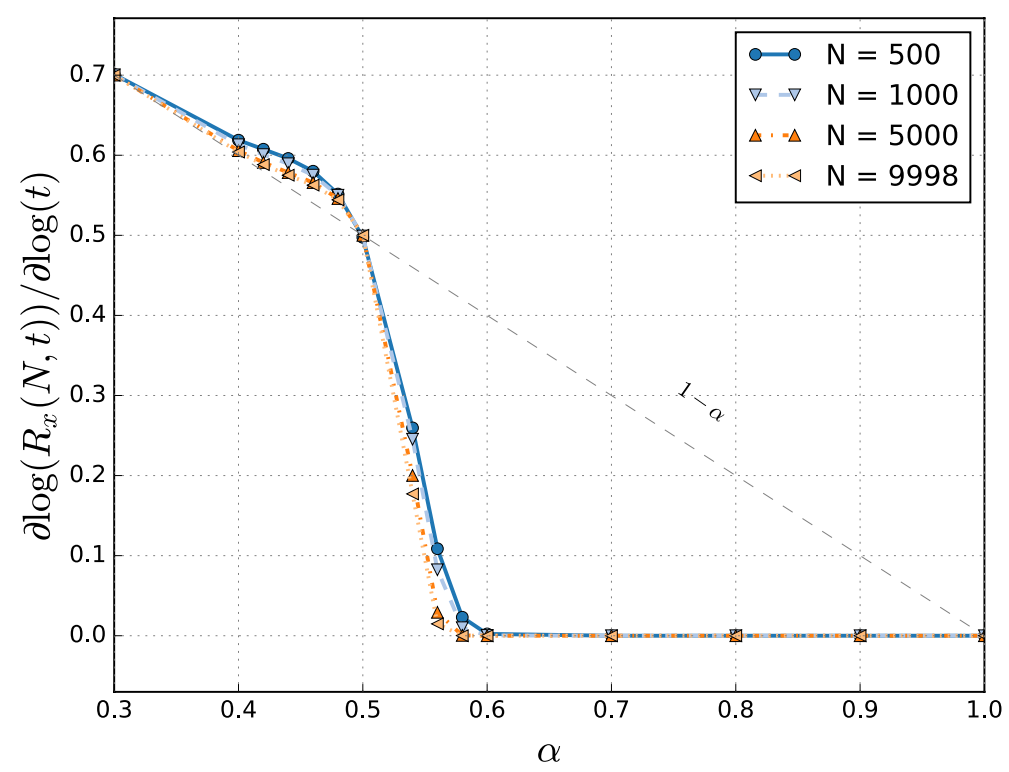

FiguRE 5.7: The exponent of the growth power-law of the tip point vs. $\alpha$ for different growth stages $N=500,1000, \cdots$. For $\alpha<1 / 2$, the tip point in the lab reference frame scales like $t^{1-\alpha}$. At around $\alpha=0.5$ the exponent sharply decrease to zero due to the sudden arrest of the tip point for $\alpha>1 / 2$. This transition becomes sharper for longer chains.

In contrast, for $\alpha>1$ the expected behavior $\sim t^{1-\alpha}$ is replaced by a rather sudden stop of the center of mass. Figure 5.8 illustrates this transition. Here, we sketch the slope of the log-log graph of the averaged position of the center of mass against $\alpha$. From this figure, the transition in the behavior of $\vec{R}_{\text {com }}$ at $\alpha=1$ is obvious. What still remains unclear, is the deviation of the slope from our expectation for $0.5<\alpha<1$, for which we cannot offer a simple physical explanation at present.

\subsubsection{Base point $R(0, t)$}

It is also worth mentioning the behavior of the dragged point at $n=0$. For $\alpha<0.5$, the dragged point in the reference frame of the center of mass moves as $t^{\alpha}$ (see Table 5.1), while the center of mass in the lab frame moves as $t^{1-\alpha}$. Therefore the motion of the dragged point in the lab frame should be dominated by the center of mass:

$$
R(0, t) \sim t^{1-\alpha}
$$

Increasing $\alpha$ beyond $1 / 2$, the dominant dynamics is the motion of the base relative to the center of mass. According to Figure 5.4, in the fast polymerization regime, the position of the base point in the reference frame of the center of mass scales like $t^{1 / 2}$, while the 


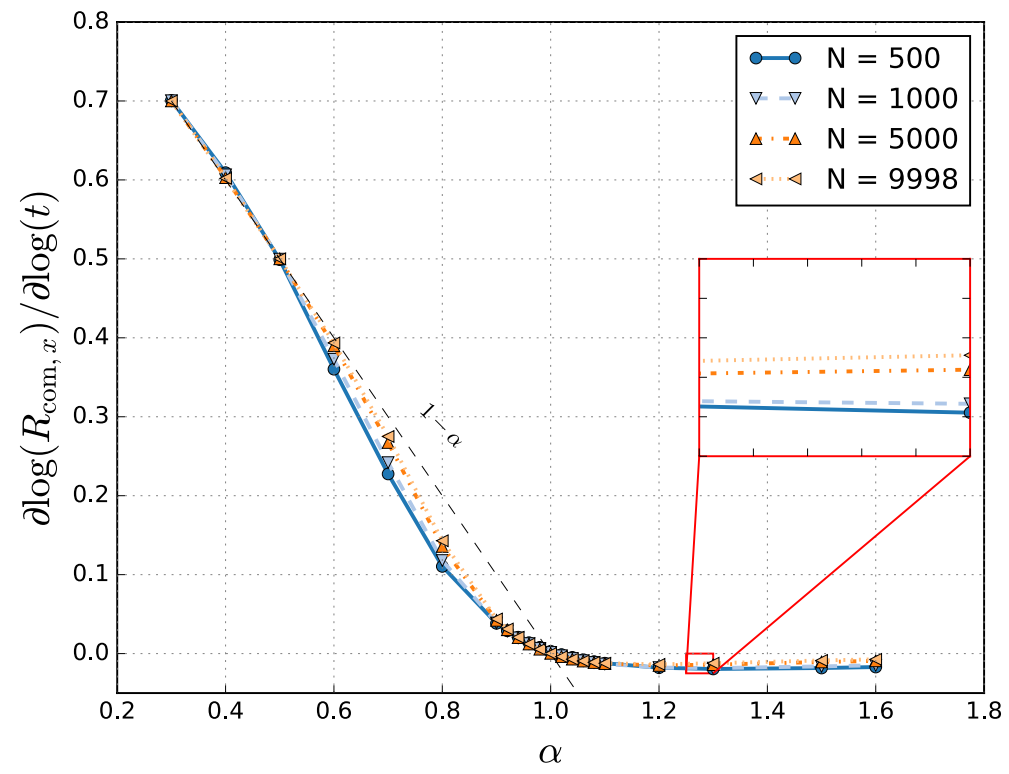

FiguRE 5.8: Logarithmic derivative of the logarithm of $\mathrm{x}$-component of the center-of-mass vs. $\alpha$. The dashed line corresponds to the simple expectation $t^{1-\alpha}$, calculated for slow polymerization regime. The inset shows the approach to zero (upper boundary of inset) for $1.25<\alpha<1.3$.

center of mass moves as $t^{1-\alpha}$. Consequently:

$$
R(0, t) \sim t^{0.5}
$$

Figures $5.9 \mathrm{a}$ and 5.9 depicts this transition.

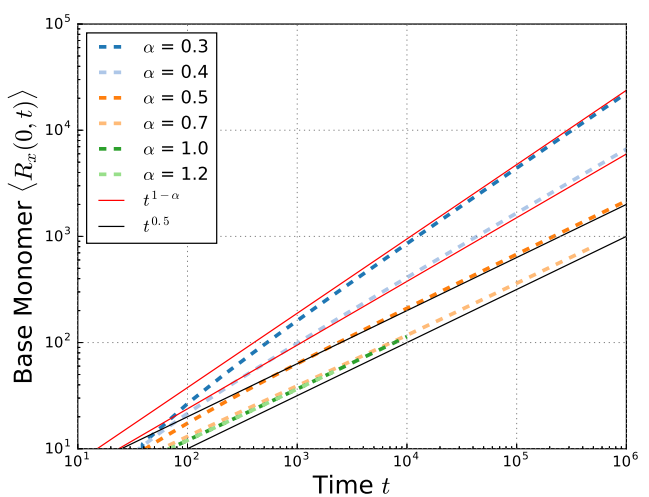

(A)

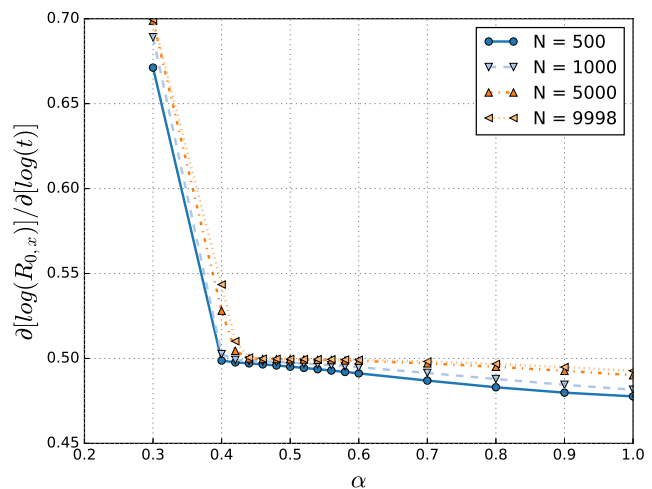

(B)

FIGURE 5.9: (a)The averaged position of the base monomer in time measured in the lab frame and (b) the exponent of the power-law growth of this quantity computed at various polymerization stages $(N=500,1000, \cdots)$ as a function of the growth exponent $\alpha$. For $\alpha<1 / 2$, the base point follows the center-of-mass, and therefore the exponent of the corresponding power-law approaches $t^{1-\alpha}$ as the chain length increases. Beyond $\alpha=1 / 2$, this exponent approaches asymptotically to the constant value $1 / 2$. 


\section{Chapter 6}

\section{Living Rouse Chain in Shear Flow}

In polymeric systems, one important scenario which deserves attention is polymers under flow. In this scenario as described by Eq.(2.16), a velocity field is imposed on solvent molecules. Due to the change in the relative velocity of polymers and solvent, the friction experienced by the polymers is changed and the stretched polymers introduce additional stresses to the fluid, which change its rheological properties. In this chapter, we will extend such studies to the case of growing chains in a shear flow.

\subsection{Some elements of rheology}

Rheology is the study of flow and deformation of materials under applied stimuli. In experiments it is common to impose an external velocity field (flow) on the material under study. The mechanical response of the body is then measured in terms of internal stresses formed by the external flow.

A polymeric liquid contains a large number of polymer chains surrounded by solvent molecules. We are interested in micro-rheology of polymeric liquids. In other words, we will study how the dynamics of single polymer molecules are affected by an external flow field. From such a microscopic description of the system, we then try to extract macroscopic flow behavior of the polymeric fluid.

\subsubsection{Steady shear flow}

From the point of view of continuum mechanics, an external distortion in a fluid can be represented by a Eulerian velocity field $v(\vec{r}, t)$, which specifies the average velocity of materials inside a small volume element $\mathrm{d} V$ at $\vec{r}$ in space and at time $t$. Note that 
throughout this chapter we will use the non-dimensionalized variables introduced in Section 2.1.3. The velocity field is a macroscopic kinetic quantity; The length scales involved in the velocity field (e.g. the dimensions of the fluid container) are much bigger than the microscopic lengths (e.g. dimension of a monomer). This implies that around a single polymer chain, which is small compared to the length scale of the flow but large compared to a single monomer, the linear approximation of the flow field is justified. Let us assume that a polymer chain is placed at an arbitrary point $\vec{r}_{0}$ (for example consider that its center of mass is placed at this point). Around this point, one can Taylor expand the velocity field as:

$$
\vec{v}(\vec{r}, t)=\vec{v}(0, t)+\kappa(0, t) \cdot \vec{r}+o(\vec{r})
$$

where $\kappa$ is the velocity gradient tensor at the origin:

$$
\hat{\kappa}:=\left[\begin{array}{lll}
\frac{\partial v_{x}}{\partial x} & \frac{\partial v_{x}}{\partial y} & \frac{\partial v_{x}}{\partial z} \\
\frac{\partial v_{y}}{\partial x} & \frac{\partial v_{y}}{\partial y} & \frac{\partial v_{y}}{\partial z} \\
\frac{\partial v_{z}}{\partial x} & \frac{\partial v_{z}}{\partial y} & \frac{\partial v_{z}}{\partial z}
\end{array}\right]
$$

In Eq.(6.1), without loss of generality, we have put the origin at point $\vec{r}_{0}=\overrightarrow{0}$. The zero ${ }^{\text {th }}$ order term in Eq.(6.1) is a translation of all the molecules inside the system as a whole. It does not change distances between molecules and therefore has no impact on internal stresses (which later on we will introduce as a measure for mechanical response of the system) in the fluid. The second term, however, shows how velocity changes from point to point in the container. It says, how materials move relative to each other. In fact, these relative motions change the distances between molecules and lead to formation of stress inside the body.

We will discuss the dynamics of growing polymers in presence of a steady shear flow. In particular, we will derive analytical expressions for the dynamics of the bonds $\vec{b}(n, t)$, which in turn can be used to calculate rheologically important observables such as the stress tensor.

Fig.(6.1) illustrates schematically the experimental setup: A polymeric liquid between two parallel plates with normal vectors along the $y$ axis is deformed by moving the upper plate parallel to another with a constant velocity $V$. Consider $h$ as the distance between two palates, the macroscopic shear rate $\kappa$ is defined as:

$$
\kappa:=\frac{V}{h}
$$

This distortion, if applied slowly enough, imposes a time independent macroscopic flow field $\vec{v}=(\kappa y, 0,0)$. From the definition in Eq.(6.2), the velocity gradient tensor for this 


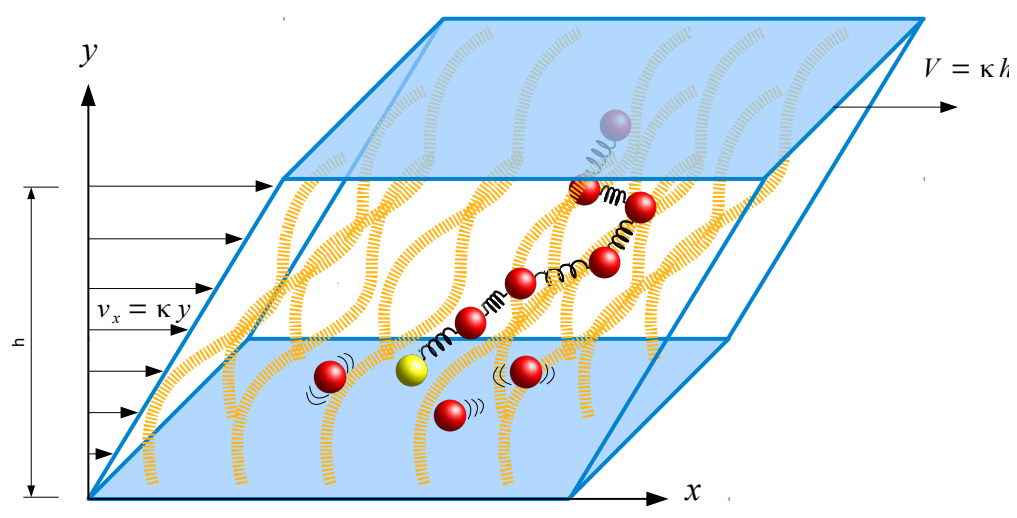

FiguRE 6.1: Living polymers under a steady shear flow. The upper plate is dragged via a constant speed $V$, while keeping the lower plate fixed. During deformation, polymer chains are growing.

steady shear flow reads:

$$
\hat{\kappa}=\left[\begin{array}{lll}
0 & \kappa & 0 \\
0 & 0 & 0 \\
0 & 0 & 0
\end{array}\right]
$$

Note that the velocity gradient $\kappa$ introduces a time-scale in the system:

$$
\tau_{f}:=\frac{1}{\kappa}
$$

This is in fact the time scale, over which the flow acts locally on the body. For a polymer under shear flow, a dumbbell initially along the $y$ axis is aligned in the direction of the flow over this time scale.

\subsubsection{Stress tensor}

Internal stresses are usually expressed by stress tensors $\hat{\sigma}[15]$; a second order tensor in Cartesian space, that allows for calculation of the force exerted on an arbitrary surface element inside the body. To define the stress tensor, consider an imaginary surface element $\mathrm{d} \vec{A}$ within the fluid, with a normal direction $\vec{n}$. Let $\mathrm{d} \vec{F}(\vec{n})$ be the total force exerted on this surface element, due to the interactions between molecules that are above the surface, and the molecules that are on the other side. By definition, the stress tensor is defined such that:

$$
\mathrm{d} \vec{F}(\vec{n})=-\hat{\sigma} \cdot \hat{n} \mathrm{~d} A
$$


The quantity $\frac{\mathrm{d} \vec{F}}{\mathrm{~d} A}$ is called traction and has the dimension of pressure. In rheology of polymeric fluids, it is useful to decompose the molecular interactions in two parts: One part is the interactions involving solvent molecules and the other is due to the interactions between monomers making up the polymers. Consequently, the stress tensor can be written as:

$$
\hat{\sigma}_{\alpha, \beta}=\hat{\sigma}_{\alpha, \beta}^{s}+\hat{\sigma}_{\alpha, \beta}^{p}
$$

If we consider a Newtonian solvent, $\sigma_{\alpha, \beta}^{s}$ reads:

$$
\hat{\sigma}_{\alpha, \beta}^{s}=\eta_{s}\left(\kappa_{\alpha, \beta}+\kappa_{\beta, \alpha}\right)+P \delta_{\alpha, \beta}
$$

The presence of polymers in the system makes an extra contribution in the stress tensor. The microscopic expression for the stress tensor of polymers can be found in standard textbooks and is given by:

$$
\sigma_{\alpha, \beta}^{p}=-\frac{1}{V} \sum_{i} \sum_{m}\left\langle F_{m, \alpha}^{i} R_{m, \beta}^{i}\right\rangle,
$$

where $F_{m}^{i}$ is the total force, exerted from other beads on the $m^{\text {th }}$ bead of the $i^{\text {th }}$ chain which is positioned at $\vec{R}_{m}^{i}$ and $\alpha, \beta$ label the Cartesian coordinates. In general, these forces may be due to bonding as well as non-bonding forces. Here, we consider a dilute solution for which we can neglect interactions between monomers of different chains. For a mono-disperse solution, the summation over chains thus becomes the factor $c_{p}$ denoting the concentration of chains ${ }^{1}$ :

$$
\sigma_{\alpha, \beta}^{p}=-c_{p} \sum_{m}\left\langle b_{m, \alpha} b_{m, \beta}\right\rangle
$$

where we considered that in a Rouse chain the intramolecular interactions is restricted only to the neighbouring beads $F_{m, \alpha}^{i}=b i_{m+1, \alpha}-b_{m, \alpha}^{i}=-k\left(2 R_{m, \alpha}^{i}-R_{m-1, \alpha}^{i}-R_{m+1, \alpha}^{i}\right)$.

The stress tensor of Eq.(6.10) can also be written in continuum form, which used in analytical approaches:

$$
\hat{\sigma}_{\alpha, \beta}^{p}=-c_{p} \int_{n}\left\langle b_{\alpha}(n, t) b_{\beta}(n, t)\right\rangle \mathrm{d} n
$$

\subsubsection{Viscometric functions}

Instead of measuring all elements of the stress tensor, it is easier to measure new observables as combinations of these elements. Such functions are called viscometric functions [72]. Important viscometric functions to study polymers under shear flow are as

\footnotetext{
${ }^{1}$ From the experimental point of view, it is more common to express Eq.(6.10) by the monomeric concentration. For a mono-disperse solution, the concentration of monomers $c_{m}$ can be obtained as $c_{m}=N c_{p}$.
} 
follows:

- Shear viscosity: is a measure that characterizes the resistance of a fluid against deformation due to the friction between fluid layers. Viscosity $\eta$ is usually expressed in terms of the shear stress:

$$
\eta=\kappa \hat{\sigma}_{x, y}
$$

where the shear rate $\kappa$ is defined as $\kappa:=\hat{\kappa}_{x, y}$.

- Normal stress differences: are associated with the anisotropy induced by applying strain in a fluid. At a microscopic level, anisotropy in a polymer molecule is signalled by the deviation of its shape from a symmetric random coil. This information is also contained in the stress tensor. To characterize such anisotropies, the normal stress differences are measured. For a stress tensor, one can define two normal stress differences, that provide comparison for the amount of stress along different axes. These can be defined as:

1. First normal stress difference: $\sigma_{x x}-\sigma y y$

2. Second normal stress difference: $\sigma_{y y}-\sigma z z$

\subsubsection{Rheology of fixed length polymers}

As mentioned before, an external stimulus like a flow field affects the internal dynamics of polymers, particularly the dynamics of the bond vectors $\vec{b}(n, t)$. On the other hand, Eqs.(6.10) and (6.11) express the macroscopically measurable stress tensor in terms of these microscopic bond vectors. It should be therefore possible to relate the applied flow to the stress tensor. This procedure is what we meant above by micro-rheology. Micro-rheology of fixed-length Rouse chains is extensively studied and the results can be found in various standard literatures (see for example [15]). In the following, we first review these results. In particular, we point out, how viscometric functions depend upon the polymer length $N$.

In presence of a steady shear flow $v=(\kappa y, 0,0)$, a particle positioned at $\left(R_{x}, R_{y}, R_{z}\right)$ feels an extra velocity equal to $\left(\kappa R_{y}, 0,0\right)$. As a consequence, the beads experience an extra friction in $x$ direction which opposes the direction of the particle velocity. Obviously, this excess of friction depends upon the position of the bead in $y$ direction. In continuum 
limit, the equation of motion for a polymer chain under a shear flow can be written as:

$$
\begin{aligned}
& \partial_{t} R_{x}(n, t)=\partial_{n}^{2} R_{x}(n, t)+\kappa R_{y}+f_{x} \\
& \partial_{t} R_{y}(n, t)=\partial_{n}^{2} R_{y}(n, t)+f_{y}
\end{aligned}
$$

The dynamics of the chain in $y$ and $z$ directions remain that of a free chain. The corresponding evolution equation for the bonds can be set up by differentiating both sides of these equations with respect to $n$. For $b_{x}$ we can write:

$$
\partial_{t} b_{x}(n, t)=\partial_{n}^{2} b_{x}(n, t)+\kappa b_{y}+\partial_{n} f_{x}
$$

In fact, $b_{y}$ can be interpreted as an additional Gaussian noise. The properties of this noise can be inferred from our results on the free chain. Note that the evolution equations for $b_{x}(n, t)$ and $b_{y}(n, t)$ are one-way coupled. In other words, $b_{x}$ depends upon $b_{y}$ but $b_{y}$ is independent from $b_{x}$. Formally, the solution for $b_{y}$ can be expressed in terms of the Green's function:

$$
b_{y}(n, t)=\int_{0}^{t} \mathrm{~d} t^{\prime} \int_{0}^{N} \mathrm{~d} n^{\prime} \partial_{n^{\prime}} G_{D}\left(n, t \mid n^{\prime}, t^{\prime}\right) f_{y}\left(n^{\prime}, t^{\prime}\right)
$$

where we have integrated over the variable $n^{\prime}$ by parts and substituted the boundary conditions for the Green's function.

the correlation function of the $b_{y} \mathrm{~s}$, which completely characterises their stochastic properties can be expressed in terms of the Green's function $G_{D}$. Using Eq.(6.16) and considering the correlation of the noise as $\left\langle f_{\alpha}(n, t) f_{\beta}\left(n^{\prime}, t^{\prime}\right)\right\rangle=2 \delta_{\alpha, \beta} \delta\left(n-n^{\prime}\right) \delta\left(t-t^{\prime}\right)$, we can write:

$$
\left\langle b_{y}(n, t) b_{y}\left(n^{\prime}, t^{\prime}\right)\right\rangle=\int_{0}^{t^{\prime}} \mathrm{d} t_{1} \int_{0}^{N} \mathrm{~d} n_{1} \partial_{n_{1}} G_{D}\left(n, t \mid n_{1}, t_{1}\right) \partial_{n_{1}} G_{D}\left(n^{\prime}, t^{\prime} \mid n_{1}, t_{1}\right),
$$

where we assumed $t>t^{\prime}$. Substituting $G_{D}$ from Chapter 2 yields:

$$
\partial_{n_{1}} G_{D}\left(n, t \mid n_{1}, t_{1}\right)=\frac{2}{N} \sum_{p=1}^{\infty} k_{p} \sin \left(k_{p} n\right) \cos \left(k_{p} n_{1}\right) e^{-k_{p}^{2}\left(t-t_{1}\right)} .
$$

Plugging this equation into Eq.(6.17) results in:

$$
\begin{aligned}
& \left\langle b_{y}(n, t) b_{y}\left(n^{\prime}, t^{\prime}\right)\right\rangle \\
& =\frac{4}{N^{2}} \sum_{p, q} k_{p} k_{q} \sin \left(k_{p} n\right) \sin \left(k_{q} n^{\prime}\right) \int_{0}^{t^{\prime}} \mathrm{d} t_{1} e^{-k_{p}^{2}\left(t-t_{1}\right)-k_{q}^{2}\left(t^{\prime}-t_{1}\right)} \int_{0}^{N} \cos \left(k_{p} n_{1}\right) \cos \left(k_{q} n_{1}\right) \mathrm{d} n_{1} .
\end{aligned}
$$


Note that $\int_{0}^{N} \cos \left(k_{p} n_{1}\right) \cos \left(k_{q} n_{1}\right) \mathrm{d} n_{1}=\frac{N}{2} \delta_{p, q}$. Using this relation and performing the summation over $q$ in Eq.(6.19) we can write:

$$
\left\langle b_{y}(n, t) b_{y}\left(n^{\prime}, t^{\prime}\right)\right\rangle=\frac{2}{N} \sum_{p=1}^{N} k_{p}^{2} \sin \left(k_{p} n\right) \sin \left(k_{p} n^{\prime}\right) \int_{0}^{t^{\prime}} \mathrm{d} t_{1} e^{-k_{p}^{2}\left(t+t^{\prime}\right)+2 k_{p}^{2} t_{1}} .
$$

If we integrate over $t_{1}$, for the long time regime $t \gg \tau_{R}$, we ultimately obtain:

$$
\left.\left\langle b_{y}(n, t) b_{y}\left(n^{\prime}, t^{\prime}\right)\right\rangle=\frac{1}{2}\left[\Theta\left(t-t^{\prime}\right) G_{D}\left(n, t \mid n^{\prime}, t^{\prime}\right)+\Theta\left(t^{\prime}-t\right) G_{(} n^{\prime}, t^{\prime} \mid n, t\right)\right] .
$$

The $x$ component of the bond vector $b_{x}(n, t)$ can be calculated by plugging Eq.(6.16) into Eq.(6.15)

$$
b_{x}(n, t)=\int_{0}^{t} \mathrm{~d} t^{\prime} \int_{0}^{N} \mathrm{~d} n^{\prime} G_{D}\left(n, t \mid n^{\prime}, t^{\prime}\right)\left[\kappa b_{y}\left(n^{\prime}, t^{\prime}\right)+\partial_{n^{\prime}} f_{x}\left(n^{\prime}, t^{\prime}\right)\right] .
$$

Here, the transient terms due to the relaxation of the initial condition have been neglected. At this point, we can express various rheologically interesting observables in terms of the Green's function:

- End to End distance squared:

$$
\begin{aligned}
\left\langle R_{x, e e}^{2}(t)\right\rangle= & \int_{0}^{N} \mathrm{~d} n \int_{0}^{N} \mathrm{~d} n^{\prime} \int_{0}^{t} \mathrm{~d} t_{1} \int_{0}^{t} \mathrm{~d} t_{2} \int_{0}^{N} \mathrm{~d} m \int_{0}^{N} \mathrm{~d} m^{\prime} \\
& \times G_{D}\left(n, t \mid m, t_{1}\right) G_{D}\left(n^{\prime}, t \mid m^{\prime}, t_{2}\right) \\
& \times\left(\kappa\left(t_{1}\right) \kappa\left(t_{2}\right)\left\langle b_{y}\left(m, t_{1}\right) b_{y}\left(m^{\prime}, t_{2}\right)\right\rangle+\left\langle\partial_{m} f\left(m, t_{1}\right) \partial_{m} f\left(m^{\prime}, t_{2}\right)\right\rangle\right)
\end{aligned}
$$

- Shear Stress:

$$
\begin{aligned}
\sigma_{x, y} & =\int_{0}^{N}\left\langle b_{x}(n, t) b_{y}(n, t)\right\rangle \mathrm{d} n \\
& =\int_{0}^{N} d n \int_{0}^{t} \mathrm{~d} t^{\prime} \int_{0}^{N} \mathrm{~d} n^{\prime} G_{D}\left(n, t \mid n^{\prime}, t^{\prime}\right) \kappa\left(t^{\prime}\right)\left\langle b_{y}(n, t) b_{y}\left(n^{\prime}, t^{\prime}\right)\right\rangle
\end{aligned}
$$

- First normal stress difference $\sigma_{x, x}-\sigma_{y, y}$ :

$$
\sigma_{x, x}-\sigma_{y y}=\int_{0}^{N} \mathrm{~d} n\left\langle b_{x}^{2}(n, t)-b_{y}^{2}(n, t)\right\rangle
$$


where:

$$
\begin{aligned}
\left\langle b_{x}^{2}(n, t)-b_{y}^{2}(n, t)\right\rangle & \\
& =\kappa^{2} \int_{0}^{t} \mathrm{~d} \tau_{1} \int_{0}^{t} \mathrm{~d} \tau_{2} \int_{0}^{N} \mathrm{~d} m_{1} \int_{0}^{N} \mathrm{~d} m_{2} \\
& G_{D}\left(n, t \mid m_{1}, \tau_{1}\right) G_{D}\left(n, t \mid m_{2}, \tau_{2}\right)\left\langle b_{y}\left(m_{1}, \tau_{1}\right) b_{y}\left(m_{2}, \tau_{2}\right)\right\rangle
\end{aligned}
$$

- Second normal stress difference $\sigma_{y, y}-\sigma_{z, z}$ : This term is equal to zero.

We can now calculate the rheological properties of fixed-length polymers using Eq.(6.20). Table 6.1 summarizes these results[73]:

\begin{tabular}{c|c} 
Observables & Results from Rouse Model \\
\hline$\left\langle R_{x, e e}^{2}\right\rangle$ & $\kappa^{2} N^{5}+N$ \\
\hline$\sigma_{x, y}$ & $\frac{\kappa N^{2}}{48}$ \\
\hline$\sigma_{x x}-\sigma_{y y}$ & $\frac{\kappa^{2} N^{4}}{1440}$
\end{tabular}

TABLE 6.1: The time- and length-dependence of various rheological observables, calculated for Rouse chains.

In the next section, we will work out the rheology of growing Rouse chains. Our analytical calculation is restricted to the slow-polymerization regime, where $N(t)=o\left(t^{0.5}\right)$. Recall that in this regime, the dynamics of the growing chain, asymptotically, can be fully mapped to the dynamics of a fixed-length Rouse chain with length 1 . The rheology of the fast polymerization regime will be analyzed numerically using the algorithm presented in Sections 3.1 and 6.3.

\subsection{Slow polymerization regime}

Here we present the rheological properties, calculated for slowly growing Rouse chains under a steady shear flow with shear rate $\kappa$. The procedure for calculating the shear stress $\hat{\sigma}_{x, y}$ and mean squared end to end distance is presented in detail.

Applying the Landau transformation given in Eq.(3.16) and time transformation in Eq.(3.21) to the system of stochastic equations for $b_{y}$ and $b_{x}$ and after balancing the 
dominating terms in the long time regime we can write:

$$
\partial_{\tau} \rho_{x}(\sigma, \tau)=\partial_{\sigma}^{2} \rho_{x}+\kappa \cdot N^{2}(\tau) \cdot \rho_{y}+f_{x}(\sigma, \tau)
$$

where $\vec{\rho}$ is defined as in Eq.(4.6). The boundary condition for a chain with two free ends is given in Eq.(2.59). The white noise $\vec{f}$ is characterized by its first and second moments which are given in Eqs.(3.23) and (3.24).

Eq.(6.31) has a nice interpretation. It shows that in the slow polymerization regime, the dynamics of a growing chain under a steady shear flow $\kappa$ can be mapped to the dynamics of a fixed length chain, subjected to an unsteady shear flow $\kappa^{*}(\tau)$, which is defined as:

$$
\kappa^{*}(\tau):=\kappa \cdot N^{2}(\tau)
$$

Let us proceed with the calculation of the shear stress. If we introduce $\vec{\beta}:=\partial_{\sigma} \vec{\sigma}$, we have:

$$
\vec{b}=\partial_{n} \vec{R}=1 / N \partial_{\sigma} \sqrt{N} \vec{\rho}=\vec{\beta} / \sqrt{N}
$$

. Thus, one can write:

$$
\hat{\sigma}_{x, y}(t)=\int_{0}^{N(t)} \mathrm{d} n\left\langle b_{x}(n, t) b_{y}(n, t)\right\rangle=\int_{0}^{1} \mathrm{~d} \sigma\left\langle\beta_{x}(\sigma, \tau(t)) \beta_{y}(\sigma, \tau(t))\right\rangle
$$

Eq.(6.33) implies that the stress tensor of the transformed chain, is exactly equal to the stress tensor of the growing chain.

Inserting Eq.(6.20) into Eq.(6.26), the stress tensor can be written in the following form:

$$
\hat{\sigma}_{x, y}(\tau)=\sum_{p=1}^{\infty} \int_{0}^{\tau} \mathrm{d} \tau^{\prime} \sigma_{p}\left(\tau, \tau^{\prime}\right)
$$

where we introduced:

$$
\sigma_{p}\left(\tau, \tau^{\prime}\right):=\frac{\kappa^{*}\left(\tau^{\prime}\right)}{2} \exp \left[-2 k_{p}^{2}\left(\tau-\tau^{\prime}\right)\right]
$$

The integration of Eq.(6.34) can not be performed exactly. Figure 6.2 depicts the $\sigma_{p}\left(\tau, \tau^{\prime}\right)$ for a fixed value of $\tau$ and for different values of $p$. Because the most important contribution to the integral comes from the integrand value near $\tau^{\prime}=\tau$, one can approximate the integral by the area of the right triangle, whose hypotenuse is tangent to the curve at point $\tau^{\prime}=\tau$. Therefore:

$$
\int_{0}^{\tau} \mathrm{d} \tau^{\prime} \sigma_{p}\left(\tau, \tau^{\prime}\right) \approx \frac{\sigma_{p}^{2}(\tau, \tau)}{\left.2 \partial_{\tau^{\prime}} \sigma_{p}\left(\tau, \tau^{\prime}\right)\right|_{\tau^{\prime}=\tau}}=\frac{\kappa^{*}(\tau)}{8 \pi^{2} p^{2}}
$$


In the end the shear stress can be calculated as:

$$
\hat{\sigma}_{x, y}(t)=\frac{\kappa \gamma^{2} t^{2 \alpha}}{48}
$$

As we see from this equation, in contrast to fixed length polymers, whose respond to a static stimuli does not depend on time (see Table 6.1), for growing polymers, the shear stress, as a mechanical response to the steady shear flow turns out to be time-dependent. This result could also be deduced from Eqs.(6.31) and (6.33). From these relations, we have seen that the shear stress of a growing chain is exactly equal to the shear stress of a fixed length chain with length 1 subjected to a time-dependent stimuli $\kappa^{*}(t)$. For a fixed length polymer under such a time-dependent flow, we expect a time-dependent response.

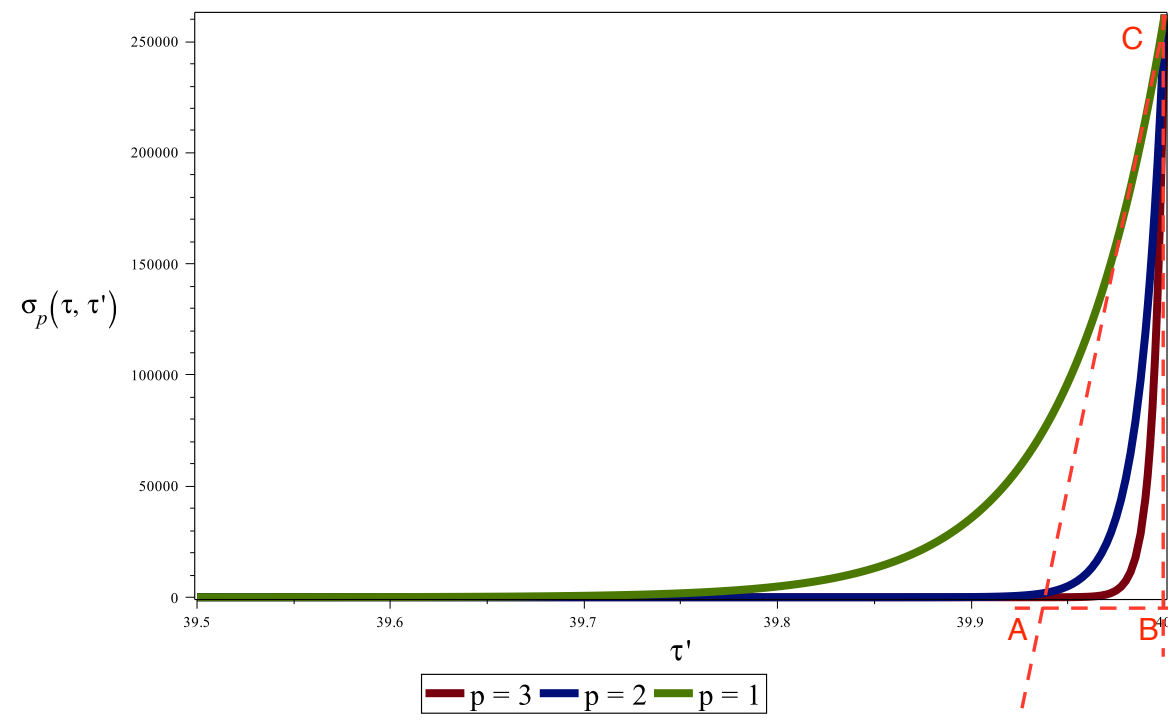

Figure 6.2: $\sigma_{p}\left(\tau, \tau^{\prime}\right)$ against $\tau^{\prime}$, for $\tau=40, \alpha=0.45$ and $\gamma=1$. The integral $\int_{0}^{\tau} \sigma_{p}\left(\tau, \tau^{\prime}\right) \mathrm{d} \tau^{\prime}$ can be approximated by the area of the right triangle, whose hypotenuse side is tangent to the curve at $\tau^{\prime}=\tau$. Figure illustrates such a triangle for $p=1$.

As another example, here we calculate the mean squared end to end distance for a slowly growing chain. Note that the end to end distance of the transformed chain and original chain are related as $\sqrt{N(t)}(\vec{\rho}(\sigma=1, \tau(t))-\vec{\rho}(\sigma=0, \tau(t)))=\vec{R}(N(t), t)-\vec{R}(0, t)$. To calculate the end to end distance of the transformed chain under an unsteady velocity gradient $\kappa^{*}(\tau)$, we insert the Green's function into Eq.(6.24):

$$
\left\langle\rho_{x, e e}^{2}(\tau)\right\rangle=\left\langle\rho_{e e, \kappa}^{2}(\tau)\right\rangle+\left\langle\rho_{e e, 0}^{2}(\tau)\right\rangle
$$


where we introduced $\left\langle\rho_{e e, \kappa}^{2}(\tau)\right\rangle$ and $\left\langle\rho_{e e, 0}^{2}(\tau)\right\rangle$ as:

$$
\begin{aligned}
\left\langle\rho_{e e, \kappa}^{2}(\tau)\right\rangle & =\int_{0}^{1} \mathrm{~d} \sigma_{1} \int_{0}^{1} \mathrm{~d} \sigma_{2} \int_{0}^{\tau} \mathrm{d} \tau_{1}^{\prime} \int_{0}^{\tau} \mathrm{d} \tau_{2}^{\prime} \int_{0}^{1} \mathrm{~d} \sigma_{1}^{\prime} \int_{0}^{1} \mathrm{~d} \sigma_{2}^{\prime} \\
& \times G_{D}\left(\sigma_{1}, \tau \mid \sigma_{1}^{\prime}, \tau_{1}^{\prime}\right) G_{D}\left(\sigma_{2}, \tau \mid \sigma_{2}^{\prime}, \tau_{2}^{\prime}\right) \kappa^{*}\left(\tau_{1}^{\prime}\right) \kappa^{*}\left(\tau_{2}^{\prime}\right)\left\langle b_{y}^{*}\left(\sigma_{1}^{\prime}, \tau_{1}^{\prime}\right) b_{y}\left(\sigma_{2}^{\prime}, \tau_{2}^{\prime}\right)\right\rangle
\end{aligned}
$$

and:

$$
\begin{aligned}
\left\langle\rho_{e e, 0}^{2}(\tau)\right\rangle & =\int_{0}^{1} \mathrm{~d} \sigma_{1} \int_{0}^{1} \mathrm{~d} \sigma_{2} \int_{0}^{\tau} \mathrm{d} \tau_{1}^{\prime} \int_{0}^{\tau} \mathrm{d} \tau_{2}^{\prime} \int_{0}^{1} \mathrm{~d} \sigma_{1}^{\prime} \int_{0}^{1} \mathrm{~d} \sigma_{2}^{\prime} \\
& \times G_{D}\left(\sigma_{1}, \tau \mid \sigma_{1}^{\prime}, \tau_{1}^{\prime}\right) G_{D}\left(\sigma_{2}, \tau \mid \sigma_{2}^{\prime}, \tau_{2}^{\prime}\right)\left\langle\partial_{\sigma_{1}^{\prime}} \eta\left(\sigma_{1}^{\prime}, \tau_{1}^{\prime}\right) \partial_{\sigma_{2}^{\prime}} \eta\left(\sigma_{2}^{\prime}, \tau_{2}^{\prime}\right)\right\rangle .
\end{aligned}
$$

$\left\langle\rho_{e e, 0}^{2}(\tau)\right\rangle$ is due to the thermal fluctuations of the chain, which is nothing other than the mean squared distance between two ends of a fixed-length polymer with length 1 , in the absence of any flow. This quantity can simply be calculated using Eq.(2.84) as:

$$
\left\langle\rho_{e e, 0}^{2}(\tau)\right\rangle=\frac{8}{\pi^{2}} \sum_{p=o d d}^{\infty} \frac{1}{p^{2}}
$$

where we used $\sum_{p=o d d}^{\infty} \frac{1}{p^{2}}=\frac{3}{4} \zeta(2)$ with $\zeta$ the standard zeta function [64]. The term $\left\langle\rho_{e e, \kappa}^{2}(\tau)\right\rangle$ in the right hand side arises due to the presence of the flow. This term will be analyzed here.

The integral in Eq.(6.39) is performed over both of the time variables $\tau_{1}^{\prime}$ and $\tau_{2}^{\prime}$. Therefore according to Eq.(6.20) we can write:

$$
\left\langle\beta_{y}\left(\sigma_{1}^{\prime}, \tau_{1}^{\prime}\right) \beta_{y}\left(\sigma_{2}^{\prime}, \tau_{2}^{\prime}\right)\right\rangle=\Theta\left(\tau_{1}^{\prime}-\tau_{2}^{\prime}\right) G_{D}\left(\sigma_{1}^{\prime}, \tau_{1}^{\prime} \mid \sigma_{2}^{\prime}, \tau_{2}^{\prime}\right)
$$

which yields:

$$
\left\langle\rho_{e e, \kappa}^{2}(\tau)\right\rangle=\sum_{p=o d d} \int_{0}^{\tau} \mathrm{d} \tau_{1}^{\prime} \kappa^{*}\left(\tau_{1}^{\prime}\right) \int_{0}^{\tau_{1}^{\prime}} \mathrm{d} \tau_{2}^{\prime} \kappa^{*}\left(\tau_{2}^{\prime}\right) \frac{1}{k_{p}^{2}} \exp \left[-2 k_{p}^{2}\left(\tau-\tau_{2}^{\prime}\right)\right]
$$

Now we can exploit the same trick shown in Figure 6.2 to approximate the integral:

$$
\left\langle\rho_{e e, \kappa}^{2}(\tau)\right\rangle \approx\left(\kappa^{*}(\tau)\right)^{2} \sum_{p=o d d} \frac{1}{k_{p}^{6}} \approx \kappa^{2} N^{4}
$$

Consequently, the mean squared end to end distance reads:

$$
\left\langle R_{x, e e}^{2}\right\rangle=\kappa^{2} N^{5}(t)+N(t)
$$

In treating polymers using the Rouse model, one has to be aware that this model is only 
applicable if the chain is not overstretched. In fact, the entropic springs between the beads allows an infinite extension of the polymer, which is an unphysical situation. We can express the overstretched regime, as the situation where:

$$
N \ll \sqrt{\left\langle\vec{R}_{e e}^{2}\right\rangle}
$$

For a system consisting of polymers with fixed lengths, by choosing the shear rate as:

$$
\kappa \ll N^{-3 / 2}
$$

we are on the safe side and can be sure, that the chain is not excessively stretched. In a growing Rouse chain, however, the situation is different. No matter how small $\kappa$ we choose, at some point the overstretched regime will arrive. Hence, our analysis presented here is only acceptable if:

$$
N(t) \ll \kappa^{-2 / 3}
$$

beyond this time regime the Rouse model fails. In Section 4.1, we remarked, that our approximate solution to the growing chain is only valid, if we are beyond a so called crossover time $t_{c}=\gamma^{\frac{2}{1-2 \alpha}}$ (see Eq.(4.2)). We can conclude, that the analysis presented in this section for slowly growing chains under shear flow are only valid within the interval $\left[t_{1}, t_{2}\right]$, where $t_{1} \gg t_{c}$ and $t_{2} \ll\left(\gamma \kappa^{2 / 3}\right)^{-\frac{1}{\alpha}}$.

The same way, we can calculate the first stress difference. Here we only present the result:

$$
\hat{\sigma}_{x x}-\hat{\sigma}_{y y} \sim N^{4}(t)
$$

Table 6.2 summarizes the time-dependence of the rheological properties of slowly growing polymers.

\begin{tabular}{c|c|c} 
Observables & Dependence on time & Dependence on $N$ \\
\hline$\left\langle R_{x, e e}^{2}\right\rangle$ & $t^{5 \alpha}$ & $\sim N^{5}(t)$ \\
\hline$\sigma_{x, y}$ & $t^{2 \alpha}$ & $\sim N^{2}(t)$ \\
\hline$\sigma_{x x}-\sigma_{y y}$ & $t^{4 \alpha}$ & $\sim N^{4}(t)$
\end{tabular}

TABLE 6.2: The time-dependence of various rheological observables, calculated for slowly growing chains. 


\subsection{Simulation algorithm}

In the discrete description of the Rouse model, the component-wise dynamical equation of motion for the beads subjected to a steady shear flow reads:

$$
\begin{aligned}
& \partial_{t} \boldsymbol{R}_{x}=-\hat{\boldsymbol{A}} \boldsymbol{R}_{x}+\kappa \boldsymbol{R}_{y}+\boldsymbol{f}_{x} \\
& \partial_{t} \boldsymbol{R}_{y}=-\hat{\boldsymbol{A}} \boldsymbol{R}_{y}+\boldsymbol{f}_{y}
\end{aligned}
$$

We assume that the chain is free at both ends, therefore the Rouse matrix $\hat{A}$ is as given in Eq.(2.13). We have simulated this system using Algorithm I described in Section 3.1 .

In simulating polymer chains under flow, as mentioned in the previous section, one has to be careful that the chain does not get overly stretched. Otherwise, the results are nothing other than artefacts and are of no value in predicting the behaviour of real polymers. For a growing chain, as we discussed in the previous section, sooner or later the polymer gets overstretched. However, by choosing an appropriate value for $\kappa$, one can provide a time interval, within which the results are justified. In our simulations, we found that $\kappa=0.0005$ avoids the overstretching regime if the chains are simulated up to $N_{\max }=10^{4}$ beads.

\subsubsection{Modification of Algorithm II}

As mentioned in that section, in cases, where the Cartesian components of the modes are correlated, the Algorithm II should be modified.

The transition probability for the conformation within the non-reacting interval $\left(t_{i}, t_{i+1}\right)$, is equivalent to the transition probability of the modes. To find this transition probability, due to the Gaussian nature of the modes, it is sufficient to calculate the first and second moments of each mode. To this aim, we first make use of the fact that each mode is independent from other modes. Therefore, the transition probability $P_{\text {Rouse }}$ can be written as the product of the transition probabilities corresponding to each mode:

$$
P_{\text {Rouse }}\left(\overrightarrow{\boldsymbol{R}}_{N_{i}}\left(t_{i+1}\right) \mid \overrightarrow{\boldsymbol{R}}_{N_{i}}\left(t_{i}^{+}\right)\right)=\prod_{p=0}^{N_{i}} P\left(\vec{X}_{p}\left(t_{i+1}\right) \mid \vec{X}_{p}\left(t_{i}^{+}\right)\right) .
$$

The shear flow in $x$ direction couples the $x$ and $y$ components. The $z$ component is independent from the other components. Hence we can write the transition probability 
for the modes as:

$$
P\left(\vec{X}_{p}\left(t_{i+1}\right) \mid \vec{X}_{p}\left(t_{i}^{+}\right)\right)=P\left(X_{p}\left(t_{i+1}\right), Y_{p}\left(t_{i+1}\right) \mid X_{p}\left(t_{i}^{+}\right), Y_{p}\left(t_{i}^{+}\right)\right) \times P\left(Z_{p}\left(t_{i+1}\right) \mid Z_{p}\left(t_{i}^{+}\right)\right),
$$

where we introduced the Cartesian components of each mode as $\vec{X}_{p}(t)=\left(X_{p}(t), Y_{p}(t), Z_{p}(t)\right)$. The calculation of the moments in the $z$ direction is exactly the same as for a free chain. To construct the probability distribution $P\left(X_{p}\left(t_{i+1}\right), Y_{p}\left(t_{i+1}\right) \mid X_{p}\left(t_{i}^{+}\right), Y_{p}\left(t_{i}^{+}\right)\right)$we need to determine the mean vector:

$$
\vec{\mu}_{p}=\left[\begin{array}{c}
\left\langle X_{p}\left(t_{i+1}\right)\right\rangle \\
\left\langle Y_{p}\left(t_{i+1}\right)\right\rangle
\end{array}\right]
$$

as well as the covariance matrix $\Sigma_{p}$ :

$$
\Sigma_{p}=\left[\begin{array}{cc}
\left\langle X_{p}^{2}\left(t_{i+1}\right)\right\rangle & \left\langle X_{p}\left(t_{i+1}\right) Y_{p}\left(t_{i+1}\right)\right\rangle \\
\left\langle X_{p}\left(t_{i+1}\right) Y_{p}\left(t_{i+1}\right)\right\rangle & \left\langle X_{p}^{2}\left(t_{i+1}\right)\right\rangle
\end{array}\right]
$$

Note that the averages in Eqs.(6.54) and (6.55) are taken over the conditional probability $P\left(X_{p}\left(t_{i+1}\right), Y_{p}\left(t_{i+1}\right) \mid X_{p}\left(t_{i}^{+}\right), Y_{p}\left(t_{i}^{+}\right)\right)$. By substituting the modes into the evolution equation for $\vec{R}$, one can write the evolution equation for the modes:

$$
\begin{array}{r}
\frac{\mathrm{d}}{\mathrm{d} t} X_{p}=c_{p} X_{p}+\kappa Y_{p}+\eta_{p, x} \\
\frac{\mathrm{d}}{\mathrm{d} t} Y_{p}=c_{p} Y_{p}+\eta_{p, y}
\end{array}
$$

These equations are supplemented by the given initial conditions $\left(X_{p}\left(t_{i}^{+}\right), Y_{p}\left(t_{i}^{+}\right)\right)$. Averaging Eqs(6.56) and (6.57) over the noise thermal noise, we can write:

$$
\begin{aligned}
\left\langle Y_{p}\left(t_{i+1}\right)\right\rangle & =Y_{p}\left(t_{i}\right) e^{-c_{p} \tau_{i}} \\
\left\langle X_{p}\left(t_{i+1}\right)\right\rangle & =X_{p}\left(t_{i}\right) e^{-c_{p} \tau_{i}}+\int_{t_{i}}^{t_{i+1}}\left[\kappa Y_{p}\left(t_{i}\right) e^{-c_{p}\left(t^{\prime}-t_{i}\right)}\right] e^{-c_{p}\left(t_{i+1}-t^{\prime}\right)} \mathrm{d} t^{\prime} \\
& =\left[X_{p}\left(t_{i}\right)+\kappa Y_{p}\left(t_{i}\right) \tau_{i}\right] e^{-c_{p} \tau_{i}}
\end{aligned}
$$

with $\tau_{i}=t_{i+1}-t_{i}$ the waiting time between two successive chemical reactions. Next we try to determine $Y_{p}^{2}\left(t_{i+1}\right)$ We multiply both sides of Eq.(6.57) by $2 Y_{p}$ and average over noise to obtain the equation of motion for $\left\langle Y_{p}^{2}\right\rangle$ :

$$
\frac{\mathrm{d}}{\mathrm{d} t}\left\langle Y_{p}^{2}\right\rangle=2 c_{p}\left\langle Y_{p}^{2}\right\rangle+2\left\langle Y_{p} \eta_{p, y}\right\rangle
$$


To solve Eq.(6.60) we need to determine $\left\langle Y_{p} \eta_{p, y}\right\rangle$. In order to find this correlation, we first write the short-time solution of $Y_{p}$ :

$$
Y_{p}\left(t_{i+1}\right)=Y_{p}\left(t_{i+1}-\delta\right)-c_{p}^{2} Y_{p}\left(t_{i+1}-\delta\right) \delta+\int_{t_{i+1}-\delta}^{t_{i+1}} \eta_{p}\left(t^{\prime}\right) \mathrm{d} t^{\prime}
$$

where $\delta \ll 1$. Then, we multiply both sides of this equation by $\eta_{p}\left(t_{i+1}\right)$ and average over this noise:

$$
\left\langle\eta_{p}\left(t_{i+1}\right) Y_{p}\left(t_{i+1}\right)\right\rangle=1
$$

Substituting this correlation into Eq.(6.60):

$$
\frac{\mathrm{d}}{\mathrm{d} t}\left\langle Y_{p}^{2}\right\rangle=2 c_{p}\left\langle Y_{p}^{2}\right\rangle+2
$$

we can find $\left\langle Y_{p}^{2}\right\rangle$ :

$$
\left\langle Y_{p}^{2}\left(t_{i+1}\right)\right\rangle=\frac{1}{c_{p}}+\left[Y_{p}^{2}\left(t_{i}^{+}\right)-\frac{1}{c_{p}}\right] .
$$

Now we are able to calculate $\left\langle X_{p}\left(t_{i+1}\right) Y_{p}\left(t_{i+1}\right)\right\rangle$. First, we multiply both sides of Eq.(6.56) by $Y_{p}$ and Eq.(6.57) by $X_{p}$ to obtain

$$
\begin{aligned}
X_{p} \frac{\mathrm{d} Y_{p}}{\mathrm{~d} t} & =-c_{p} X_{p} Y_{p}+X_{p} \eta_{p, y} \\
Y_{p} \frac{\mathrm{d} X_{p}}{\mathrm{~d} t} & =-c_{p} X_{p} Y_{p}+\kappa Y_{p}^{2}+Y_{p} \eta_{p, x} .
\end{aligned}
$$

Then we add these two equations and average over the thermal noise to obtain the equation of motion for $\left\langle X_{p} Y_{p}\right\rangle$ :

$$
\frac{\mathrm{d}\left\langle X_{p} Y_{p}\right\rangle}{\mathrm{d} t^{\prime}}=-2 c_{p}\left\langle X_{p} Y_{p}\right\rangle+\kappa\left[\frac{1}{c_{p}}+\left(Y_{p}(t)-\frac{1}{c_{p}}\right) e^{-2 c_{p}\left(t^{\prime}-t_{i}\right)}\right]
$$

This equation can be solved as:

$$
\begin{aligned}
\left\langle X_{p}\left(t_{i+1}\right) Y_{p}\left(t_{i+1}\right)\right\rangle & =\left[X_{p}\left(t_{i}^{+}\right) Y_{p}\left(t_{i}^{+}\right)-\frac{\kappa}{2 c_{p}^{2}}\right] e^{-2 c_{p} \tau_{i}} \\
& +\kappa\left[Y_{p}^{2}\left(t_{i}\right)-\frac{1}{c_{p}}\right] \tau_{i} e^{-2 c_{p} \tau_{i}} \\
& +\frac{\kappa}{2 c_{p}^{2}} .
\end{aligned}
$$


Finally, similar to what we have obtained for $\left\langle Y_{p}^{2}\right\rangle$ and $\left\langle X_{p} Y_{p}\right\rangle$, we can calculate $\left\langle X_{p}^{2}\right\rangle$ :

$$
\begin{aligned}
\left\langle X_{p}^{2}\left(t_{i+1}\right)\right\rangle= & {\left[X_{p}^{2}\left(t_{i}\right)-\frac{\kappa^{2}}{2 c_{p}^{3}}+\frac{1}{c_{p}}\right] e^{-2 c_{p} \tau_{i}} } \\
& +2 \kappa\left[X_{p}\left(t_{i}\right) Y_{p}\left(t_{i}\right)-\frac{\kappa}{2 c_{p}}\right] \tau_{i} t e^{-2 c_{p} \tau} \\
& +\kappa^{2}\left[Y_{p}^{2}\left(t_{i}\right)-\frac{1}{c_{p}}\right]\left(\tau_{i}\right)^{2} e^{-2 c_{p} \tau_{i}} \\
& +\frac{\kappa^{2}}{2 c_{p}^{3}}+\frac{1}{c_{p}} .
\end{aligned}
$$

We can now construct the two dimensional Gaussian probability distribution with the help of $\vec{\mu}_{p}$ and $\hat{\Sigma}_{p}$ and draw the random modes $\left(X_{p}\left(t_{i+1}\right), Y_{p}\left(t_{i+1}\right)\right)$ from this probability. These random modes can then transform back to the conformation $\overrightarrow{\boldsymbol{R}}_{N_{i}}\left(t_{i+1}\right)$.

\subsection{Coil - stretch transition}

Figure 6.3a depicts the mean-squared end to end distances $\left\langle R_{e e}^{2}\right\rangle$ of growing chains in presence of flow as a function of chain length $N$. At the beginning, all chains start with a random coil conformation, where the mean squared end to end distance scales as $N(t)$ for all chains. For each curve, there exist a transition point at a certain length $N_{c}(\alpha)$, beyond which the mean squared end to end distance scales faster than $N$. In this phase, the polymers start to align to the flow. For $\alpha<1 / 2$, this quantity grows as $N(t)^{5}$, which is in accordance with our analytical result in Table 6.2. Increasing $\alpha$ beyond $1 / 2$, the mean squared end to end distance grows slower than $N^{5}$. Another striking point is that while in the slow polymerization regime the transition length $N_{c}$ does not depend upon $\alpha$, in the fast growth regime the $N_{c}$ increases with $\alpha$.

Figure $6.3 \mathrm{~b}$ illustrates the same quantity as a function of $t$. One immediately finds that for fast growing polymers, the transition from coil to stretch tends to happen at a $t_{c}$, which is independent of the growth exponent $\alpha$. Also noticeable is that the meansquared end to end distance for all fast growing chains in the stretched phase scale as $t^{2.5}$.

This behavior is also observed for the first normal stress difference $\sigma_{x x}-\sigma_{y y}$. Looking at Figure 6.4a, for $\alpha<0.5$, again the results are in a good accordance with the analytical calculations obtained in Section 6.2. For $\alpha>1 / 2$, the first normal stress difference grows slower than the slowly polymerizing chains, which means that the conformation is more symmetric in the $x y$ plane. This result is quite consistent with the end to end distance in the stretched regime shown in Figure 6.3a. 


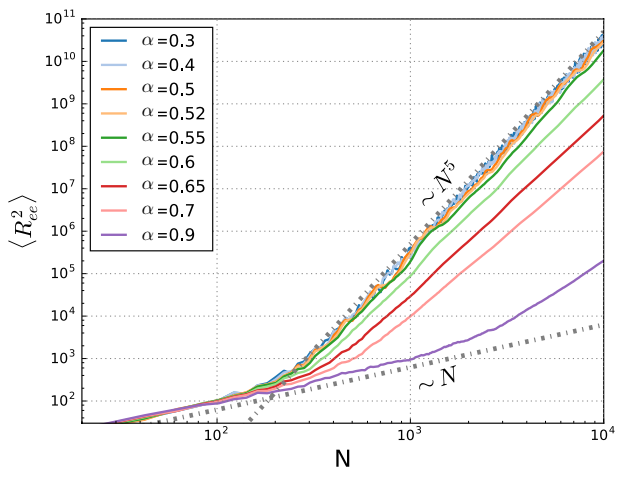

(A)

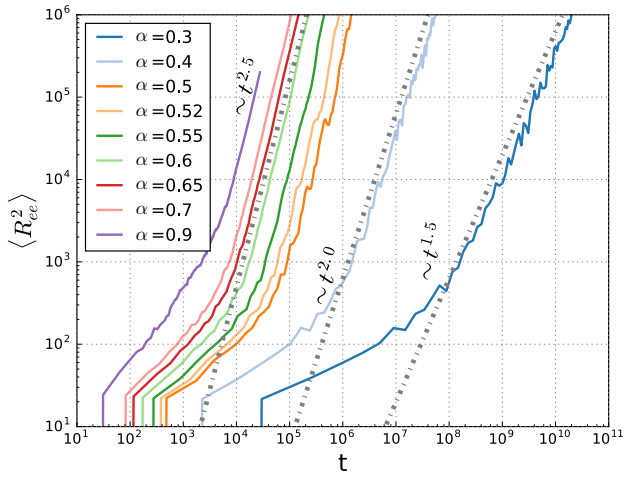

(B)

FiguRE 6.3: (A) The mean-squared end to end distance of growing chains under a steady shear flow as a function of chain length $N$. In the short time regime, all chains have random coil conformations with $\left\langle R_{e e}^{2}\right\rangle \rightarrow N(t)$. As time proceeds they start to stretch and the mean-squared end to end distance grows faster than $N$ for all chains. For growth with exponents $\alpha<1 / 2$, the transition from coil to stretch happens at universal a cross-over length, which is independent of $\alpha$ and the mean-squared end to end distance scales like $N^{5}$. Beyond $\alpha=0.5$, the cross-over length increases with $\alpha$. Increasing $\alpha$ beyond 0.5 , the exponent of this power-law decreases with $\alpha$. (B) The mean-squared end to end distance as a function of time $t$. For $\alpha>1 / 2,\left\langle R_{e e}^{2}\right\rangle \rightarrow t^{5 / 2}$ and the cross-over time from random coil to stretch approaches to a universal value independent of $\alpha$.

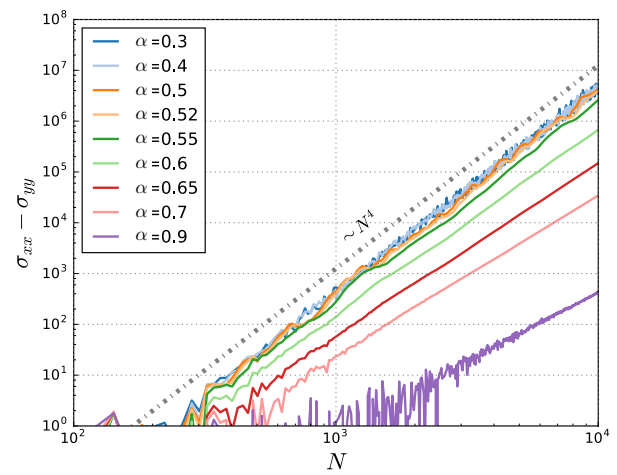

(A)

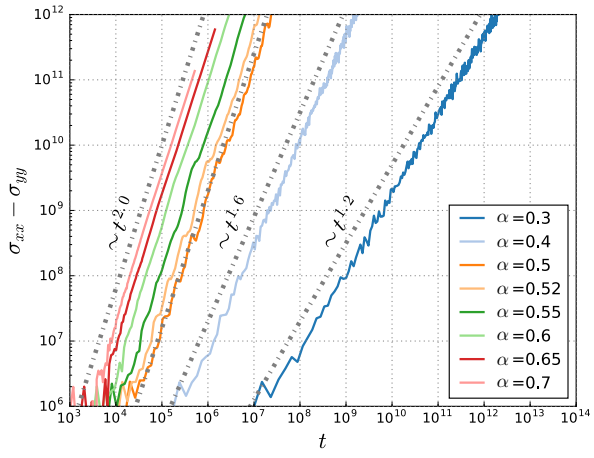

(B)

Figure 6.4: (A) The first normal stress difference $\sigma_{x x}-\sigma_{y y}$ of growing chains under a steady shear flow as a function of chain length $N$. For $\alpha<1 / 2$, the first normal stress difference grows as the power-law $N^{4}$, independent of the growth exponent $\alpha$. Beyond $\alpha=1 / 2$, the first normal stress difference grows slower than $N^{4}$ implying a more symmetric shape in the $\mathrm{x}-\mathrm{y}$ plane compared to the slowly growing chains. (B) The same quantity as in (A) as a function of time $t$. For $\alpha>1 / 2$ the first normal stress difference grows as $t^{2}$.

To see how the first normal stress difference scales in the fast polymerization regime, we sketch in Figure $6.4 \mathrm{~b}$ this quantity with respect to $t$. For $\alpha>1 / 2$, the first normal stress difference, irrespective the value of $\alpha$ scales as $t^{2}$.

Another interesting rheological property that we discussed in Section 6.1 is the shear stress. Figure 6.5a depicts this quantity as a function of chain length, and in Figure 
$6.5 \mathrm{~b}$ we sketched the same quantity as a function of time. For slowly growing chains the shear stress scales as $N^{2}(t)$ which is in agreement with the result presented in Table 6.2. According to Figure $6.5 \mathrm{~b}$, in the fast polymerization regime regardless of the growth exponent, the shear stress grows almost linearly with time.

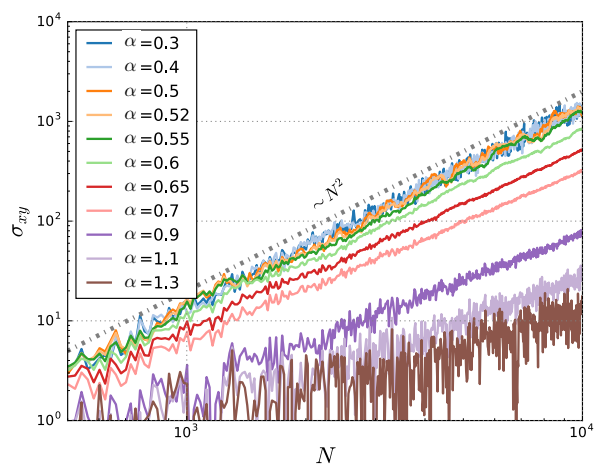

(A)

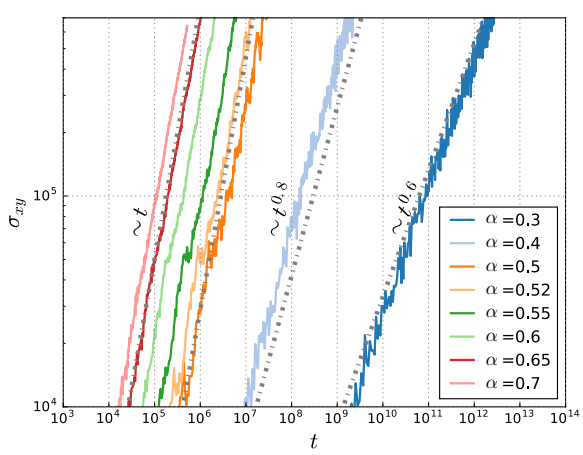

(B)

Figure 6.5: (A) Shear stress (viscosity) of growing chains under steady shear flow as a function of chain length $N$. For $\alpha<1 / 2$, the shear stress grows as $N^{2}$. Increasing $\alpha$ from $1 / 2$, a small deviation in the growth exponent of the shear rate can be observed. (B) Shear stress as a function of time $t$. For $\alpha>1 / 2$ the shear stress grows like $t$ independent of $\alpha$.

As we have seen from simulation results, one can observe an obvious transition in the mechanical response of polymeric systems to an external shear flow by passing from the slow to the fast polymerization regime. In the following, we try to explain this rheological transition.

For a fixed-length polymer under steady shear flow, there are two competing external perturbations acting on the chain. One is the thermal fluctuations, that try to equilibrate the chain to the random coil conformation. The other is the force due to the flow field, which stretches the chain. We have mentioned in Section 6.1, that the shear flow applies a local drag over the time scale $\tau_{f}=1 / \kappa$, while thermal fluctuations coils the chain over $\tau_{R}$. If the relaxation process is faster than drag due to the flow, the chain maintains its coiled conformation. On the other hand, if the shear flow is strong enough, such that $\tau_{f}<\tau_{R}$, the chain starts to stretch along the flow. This conformational transition is called coil - stretch transition. Note that for fixed-length chains $\tau_{R}$ is constant and therefore the transition from random coil to stretch conformation arises only by tuning $\kappa$ from small to large values.

For growing chains, in contrast fixed length chains, the time-scale of the coiling process is time dependent. Recall that the dynamics of tension on the chain obeys the evolution equation (6.15). Note that the dynamics of $b_{y}$ is that of the free chain and thus we can make use of the results of Chapter 4. In particular, there are two sources of $b_{y}$ fluctuations namely thermal fluctuations and fluctuations due to growth. It is these 
fluctuations which enter the equation for $b_{x}$ and drive the tension due to the shear flow. Whereas the thermal fluctuations may appear everywhere along the chain, the $b_{y}$ fluctuations due to growth are located at the tip. These latter fluctuations act as a localized source of tension for the $b_{x}$ and to stretch the whole chain this tension has to propagate along the chain diffusively. Thus the slowest process complete stretching is $t^{1 / 2}$ spreading of tension for $\alpha>1 / 2$ and the time-scale corresponding to this propagation along this comportment of the chain is indeed $t$. For $\alpha<1 / 2$ the $b_{y}$ fluctuations are dominated by thermal fluctuations which acts over the time scale $\tau_{R}(t) \sim N^{2}(t)$.

Because the coiling timescales for both of these mechanisms grows with time, even for a weak shear flow, the transition from the random coil to stretch conformation is inevitable for both slow and fast growth regimes. This is confirmed from Figure 6.3b. We can compare the timescale of the flow and this timescale by defining the ratio of these two as the Weissenberg number[74] :

$$
W i:=\frac{\text { coiling timescale }}{\tau_{f}},
$$

and the coil - stretch transition happens as $W i \sim 1$. For slowly growing chains, the coiling timescale depends only on the chain length $N(t)$. The transition length $N_{c}$ can be obtained as:

$$
N_{c}=\frac{1}{\sqrt{\kappa}},
$$

which is independent of the growth exponent $\alpha$. Because the tension propagates along the whole chain, the dynamics of stretching behaves like in a fixed chain of length $N(t)$ (see Table 6.2). Figure 6.6 depicts snapshots of the growing polymers under a steady shear flow. These snapshots are captured once the chain lengths reached $N=2000$ monomers and $N=5000$ monomers. In the short-time regime $(N=2000)$, all polymers have a coiled conformation. In the later time $(N=5000)$, all slowly growing chains become stretched, while those in the fast polymerization regime still preserve their coil conformation.

On the other hand, for fast growing chains, transition should take place at:

$$
t_{c}=\frac{1}{\kappa}
$$

a transition time which is independent of $\alpha$. In this case, the length, over which the localized tension at the tip propagates, scales as $t^{1 / 2}$ and hence in the stretching process, the chain behaves as a fixed length polymer of length $t^{1 / 2}$. Table 6.3 summarizes our hypothesis about growing chains in the fast growth regime, which are in accordance with the simulation results illustrated in Figures 6.3 to 6.5. 


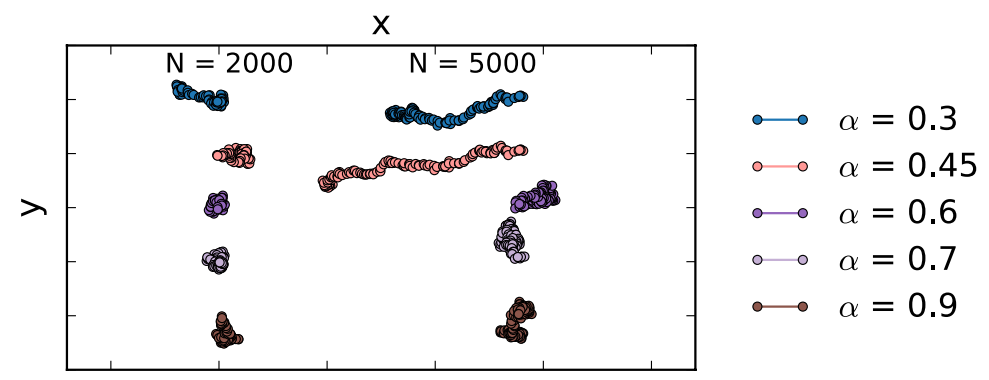

FIGURE 6.6: Snapshots from different chains growing with different growth exponents under a steady shear flow with the shear rate $\kappa=0.0005$. These snapshots have been captured in an early growth stage $(N=2000)$ and at a later time $(N=5000$ beads $)$. In the early stage, all chains have random coil conformation. In the late growth stage, the slowly growing chains with $\alpha<1 / 2$ become stretched in the flow field. The fast growing chains with $\alpha>1 / 2$, preserve almost the coil conformation.

\begin{tabular}{c|c|c} 
Observables & Dependence on time & Dependence on $N$ \\
\hline$\left\langle R_{x, e e}^{2}\right\rangle$ & $\sim t^{\frac{5}{2}}$ & $N^{\frac{5}{2 \alpha}}$ \\
\hline$\sigma_{x, y}$ & $t$ & $N^{\frac{1}{\alpha}}$ \\
\hline$\sigma_{x x}-\sigma_{y y}$ & $t^{2}$ & $N^{\frac{2}{\alpha}}$
\end{tabular}

TABLE 6.3: The time-dependence of various rheological observables, calculated for fast growing chains 


\section{Chapter 7}

\section{Summary and Conclusion}

In this thesis we studied the conformational dynamics of polymers, undergoing irreversible chain growth polymerization reactions. We discussed that the coupling between both aspects of the polymer dynamics, namely the evolution of the polymer chain and the conformational dynamics of the polymer is particularly important if the reaction timescale is comparable to the polymer relaxation timescale.

In Section 2.1, we first introduced the well-studied Rouse model for polymers of fixed length. Next in Section 2.4 the polymerization reaction has been described by a Markovian stochastic process. Finally in Section 2.5 we extended the Rouse model to situations of ongoing chain growth. Neglecting the impact of the polymer conformation on the growth, we presented discrete model for the conformational dynamics of growing chains (see Section 2.5.2). The continuous version of the model has been presented in Section 2.5.3. We neglected the chemical noise corresponding to the stochastic nature of the chemical reaction and considered the chain length to grow as a pure power-law $N(t)=\gamma t^{\alpha}$. The evolution equation of the conformation of the growing chain has then been formulated as a moving boundary value problem (see Eqs. (2.105) and (2.106)).

Based on the Brownian dynamics simulation method, in Section 3.1 we have set up a new numerical algorithm to generate conformational trajectories of growing Rouse chains. The advantage of this algorithm compared to explicit integration schemes such as Euler method has been discussed in Sections 3.1.2 and 3.1.3. Next, in Section 3.2, we introduced a collection of analytical tools required to analyse solutions of the moving boundary value problem.

In Chapter 4, we discussed the behaviour of a growing polymer, which is free at both ends. We identified two growth regimes, $0<\alpha<1 / 2$ (slow growth) and $1 / 2<\alpha$ (fast growth) separated at $\alpha=1 / 2$ (balanced growth). We discussed, that growing 
chains exhibit qualitatively different conformational properties, depending on the growth regime. Slowly growing polymers show stretched exponential relaxation (see Figure 4.1) and sub-diffusive center of mass motion with $\left\langle\vec{R}_{c o m}^{2}(t)\right\rangle \sim t^{1-\alpha}$. In contrast, in the fast growth regime, the chains never relax to the quasi-steady state. For the center of mass in this regime, we derived $\left\langle\vec{R}_{\text {com }}^{2}(t)\right\rangle \sim t^{\alpha}$, which is sub-diffusive for $1 / 2<\alpha<1$ and super-diffusive for $\alpha>1$ (Figure 4.3 left panel). We have also observed transitions in the segmental motion of growing chains by passing from the slow to the fast growth regime (see Figures 4.2 and 4.3 right panel). In Section 4.5, we gave a simple explanation to understand the basic physical mechanism of these transitions: A racing duel between the tension propagation and the polymer growth. While any disturbance on an existing monomer propagates diffusively as $t^{1 / 2}$ along the chain, the tip point keeps distance from the disturbed point as $t^{\alpha}$. For $\alpha<1 / 2$, we thus expect that the propagating front reaches the growing end, while for $\alpha>1 / 2$ it does not. In fact, in the fast polymerization regime, the tip point is causally disconnected from the existing monomers in the chain (see Figure 4.4).

In Chapter 5, we further studied the tension propagation mechanism along growing chains by considering a growing polymer dragged from its non-growing end. In the slow polymerization regime, the disturbance due to the external force spreads along the whole chain, even for longest times. In contrast in the fast growth regime, polymer growth is faster than the propagation of tension and an increasing fraction of the bonds remains force free. As a consequence, at $\alpha=1 / 2$, we observed transitions in the scaling of the internal distances of the chain (see Table 5.1). Apart from transitions at $\alpha=1 / 2$, Figure 5.8 pinpoints another dynamical transition at $\alpha=1$ in the behaviour of the center of mass: For $\alpha>1$, we observed a sudden stoppage in the average position of the center of mass.

The rheology of polymeric fluids consisting of growing chains was considered in Chapter 6. Using the perturbation method explained in Section 3.2.3, important rheological properties of slowly growing chains have been calculated. From the numerical results, we found that the coil-stretch transition for all growing chains is inevitable. However, this transition is characterized differently in different growth regimes. While for $\alpha<1 / 2$, the transition happens at a universal cross-over length $N_{c}$ (see Figure 6.3a), in the fast growth regime, the coil-stretch transition point is located at a universal time $t_{c}$ (Figure $6.3 \mathrm{~b})$. In addition to that, we found that the rheological properties such as viscosity, obey a different scaling dependent on the polymerization regime. These transitions have been attributed to the origin of the coiling, which competes the stretching due to the flow. Whereas in the slow regime the dominant process which coils the chain is thermal fluctuations, in the fast growth regime the newly produced coils at the tip of the chain due to the growth are responsible for the overall conformation of the chain. 
In conclusion, we have shown that the coupling of the simplest chain dynamics with the simplest growth reactions leads to rich and new behaviour, which can be understood from a simple physical mechanism. This argument is expected to be transferable to more complicated chain models. For instance, for stiff or semi-flexible polymers, the tension propagation evolves sub-diffusively as $t^{1 / 4}$ for longest times and $t^{1 / 8}$ for shorter times[75, 76], and therefore we expect to observe transitions at $\alpha=1 / 4$ and $\alpha=1 / 8$.

The model we introduced in this thesis can easily be applied to other scenarios, such as a growing chain fixed in space at one or both ends or the dynamics of growing polymer rings. It can also be applied to shrinking chains, which provides an alternative starting point to the problem of translocation of a polymer chain through a nanopore[70, 71]. 


\section{Appendix A}

\section{First Correction for the Regular Perturbation Method}

In this appendix, we present the first order solution to the perturbation problem given in Eq.(4.3). For simplicity, we consider that the chain is clamped at both ends, therefore the boundary condition for this problem reads:

$$
\begin{aligned}
\vec{R}(0, \tau) & =0 \\
\vec{R}(1, \tau) & =0
\end{aligned}
$$

An interested reader may work out different observables calculated in presence of the noise, and compare the corrections terms. Here, however, we neglect the noise and compute the average of the conformation over the thermal noise. Therefore the main equation of motion reads:

$$
\partial_{\tau}\langle\vec{R}\rangle=\partial_{\sigma}^{2}\langle\vec{R}\rangle+\partial_{\tau} \ln (N) \sigma \partial_{\sigma}\langle\vec{R}\rangle
$$

which is an homogeneous partial differential equation. If we had the noise term present in the system, the transformation given in Eq.(4.6) could be helpful. Here we do not need such transformation.

As we see in Eq.(A.3), from the method of dominant balances, for $\alpha<0.5$, we can split the main operator as $\mathcal{L}=\mathcal{E}+\mathcal{F}$ in the following form:

$$
\begin{array}{r}
\mathcal{E}_{\sigma, \tau}=\partial_{\tau}-\partial_{\sigma}^{2} \\
\mathcal{F}_{\sigma, \tau}=\partial_{\tau} \ln (N) \sigma \partial_{\sigma}
\end{array}
$$


To simplify our calculations, let us take the following initial condition for the conformation:

$$
\left\langle\vec{R}\left(\sigma, \tau_{0}\right)\right\rangle=\sin (\pi \sigma) \vec{e}_{x}
$$

The zeroth order perturbation solution reads:

$$
\vec{R}_{0}(\sigma, \tau)=\int_{0}^{1} \mathrm{~d} \sigma G\left(\sigma, \tau \mid \sigma^{\prime}, \tau_{0}\right) \sin \left(\pi \sigma^{\prime}\right)
$$

where the Green's function for the diffusion equation with Dirichlet boundary condition is given in Section 2.3.3. Plugging this Green's function leads to:

$$
\vec{R}_{0}=\sin (\pi \sigma) e^{-\pi^{2} \tau} \vec{e}_{x}
$$

Now we try to calculate the first correction. According to Eq.(3.33), we can write:

$$
\vec{R}_{1}=-\int_{\tau_{0}}^{\tau} \mathrm{d} \tau^{\prime} \int_{0}^{1} \mathrm{~d} \sigma^{\prime} G\left(\sigma, \tau \mid \sigma^{\prime}, \tau^{\prime}\right) \mathcal{F}_{\sigma^{\prime}, \tau^{\prime}} \vec{R}_{0}\left(\sigma^{\prime}, \tau^{\prime}\right)
$$

Note that:

$$
\mathcal{F}_{\sigma^{\prime}, \tau^{\prime}} \vec{R}_{0}\left(\sigma^{\prime}, \tau^{\prime}\right)=e^{-\pi^{2} \tau^{\prime}} \partial_{\tau^{\prime}} \ln (N) \sigma \pi \cos (\pi \sigma) \vec{e}_{x}
$$

We can substitute this relation into Eq.(A.9) and integrate over $\sigma^{\prime}$ to obtain:

$$
\vec{R}_{1}=\sum_{p} \frac{(-1)^{p}}{p^{2}-1} \sin (p \pi \sigma) e^{-(p \pi)^{\tau}} \int_{\tau_{0}}^{\tau} \partial_{\tau^{\prime}} \ln (N) e^{\pi^{2}\left(p^{2}-1\right) \tau^{\prime}} \mathrm{d} \tau^{\prime}
$$

The integral can be approximated using the method explained in Section 6.2. Finally, we will have:

$$
\vec{R}_{1} \sim \partial_{\tau} \ln (N) e^{-\pi^{2} \tau}
$$

which means that:

$$
\vec{R}_{1} \sim t^{2 \alpha-1} \vec{R}_{0}
$$

As we see, for $\alpha<0.5$, in the long time regime, $\vec{R}_{1}=o\left(\vec{R}_{0}\right)$, which means that our guess for determining the controlling operator was consistent. 


\section{Appendix B}

\section{A useful transformation}

In this appendix, we introduce a useful transformation that can be utilized in solving diffusion-drift equations. In particular, we apply this transformation to the dynamical equation:

$$
\partial_{t} Y(x, t)=c(t) \partial_{x}^{2} Y(x, t)+d(t) x \partial_{x} Y+\Psi(x, t) .
$$

As we see, the differential operator above is very similar to that of our original driftdiffusion equation, which is given in Eq.(3.22), apart from the time dependent coefficients, which here, we have chosen them as arbitrary functions of time. Let us introduce the new variable $\vec{y}(x, t)$ as:

$$
\begin{aligned}
& Y(x, t)=h(x, t) y(x, t) \\
& \Psi(x, t)=h(x, t) \psi(x, t)
\end{aligned}
$$

where $h(x, t)$ has the following form:

$$
h(x, t)=s(t) e^{a(t) x^{2}}
$$

To transform $Y \rightarrow y$ in Eq.(B.1), the following relations are required:

$$
\begin{aligned}
\partial_{t} h(x, t) & =\left[\frac{\partial_{t} s(t)}{s}+\partial_{t} a(t) x^{2}\right] h(x, t) \\
\partial_{x} h(x, t) & =2 \sigma a(t) h(x, t) \\
\partial_{x}^{2} h(x, t) & =\left[2 a(t)+4 \sigma^{2} a^{2}(t)\right] h(x, t)
\end{aligned}
$$


Now we can substitute these relations into Eq.(B.1) to obtain:

$$
\begin{aligned}
\partial_{t} y= & {\left[2 a(t) c(t)-\frac{\partial_{t} s(t)}{s(t)}\right] y } \\
& +\left[4 a^{2}(t) c(t)+2 a(t) d(t)-\partial_{t} a(t)\right] x^{2} y \\
& +[4 a(t) c(t)+d(t)] x \partial_{x} y \\
& +c(t) \partial_{x}^{2} y \\
& +\psi(x, t)
\end{aligned}
$$

Now we can determine $a(t)$, such that the convection term drops:

$$
a(t)=-\frac{d(t)}{4 c(t)}
$$

We can simplify even one step further by specifying $s(t)$ :

$$
s(t)=\exp \left[-\frac{1}{2} \int_{t_{0}}^{t} d\left(t^{\prime}\right) \mathrm{d} t^{\prime}\right]
$$

Consequently, the drift-diffusion equation converts to:

$$
\partial_{t} y=c(t) \partial_{x}^{2} y+\frac{d(t)}{4 c(t)}\left[\partial_{t} \ln \left(\frac{d}{c}\right)-d\right] x^{2} y+\psi
$$

As we see, the drift-diffusion Eq.(B.1) is now changed into a diffusion equation with an extra diagonal term. If one succeeds to find the Green's function $\tilde{\mathcal{G}}\left(x, t \mid x^{\prime}, t^{\prime}\right)$ of the operator given in Eq.(B.16), then writing the Green's function of the original equation (B.1) is not a big deal:

$$
\mathcal{G}\left(x, t \mid x^{\prime}, t^{\prime}\right)=h(x, t) \tilde{G}\left(x, t \mid x^{\prime}, t^{\prime}\right)\left[h\left(x^{\prime}, t^{\prime}\right)\right]^{-1}
$$

and therefore the solution can simply be expressed in terms of integrations over this Green's function. Let us plug the coefficients of Eq.(3.20):

$$
\begin{array}{r}
c(t)=\frac{1}{N^{2}(t)} \\
d(t)=\frac{\dot{N}}{N} \\
s(t)=\frac{1}{\sqrt{N}} \\
a(t)=-\frac{\dot{N} N}{4}
\end{array}
$$


and therefore:

$$
\frac{d(t)}{4 c(t)}\left[\partial_{t} \ln \left(\frac{d}{c}\right)-d\right]=\frac{\ddot{N} N}{4} .
$$

If we consider a power-law growth $N(t)=\gamma t^{\alpha}$, then for two cases, Eq.(B.16) simplifies: 1) For the balanced polymerization regime, where $\alpha=0.5$, the coefficient of the diagonal operator scales as $N^{-2}$ and therefore applying the time transformation given in Eq.(3.21), the time dependence of all coefficients drop out. 2) for $\alpha=1$, where the diagonal term vanishes and we are left with a simple diffusion equation. In the next two sections, we will propose an exact solution for these two cases. Before that, one thing still remains to be done, namely the transformation of the boundary condition. Note the dependence of the transformation factor $h(x, t)$ introduced above on $x$. Suppose that $0 \leq x \leq l$. This dependence can change the nature of the boundary condition as follows:

- Dirichlet boundary condition: We have $Y(x, t)=h(x, t) y(x, t)$. therefore:

$$
\left\{\begin{array} { l } 
{ Y ( x = 0 , t ) = g _ { 0 } ( t ) } \\
{ Y ( x = l , t ) = g _ { l } ( t ) }
\end{array} \Rightarrow \left\{\begin{array}{l}
y(x=0, t)=[h(0, t)]^{-1} g_{0}(t) \\
y(x=l, t)=[h(l, t)]^{-1} g_{l}(t)
\end{array}\right.\right.
$$

- von Neumann boundary condition: We have $\frac{\partial Y}{\partial x}=\frac{\partial h}{\partial x} y(x, t)+h(x, t) \frac{\partial y}{\partial x}$. therefore:

$$
\left\{\begin{array} { l l } 
{ \frac { \partial Y } { \partial x } | _ { x = 0 } } & { = g _ { 0 } ( t ) } \\
{ \frac { \partial Y } { \partial x } | _ { x = l } } & { = g _ { l } ( t ) }
\end{array} \Rightarrow \left\{\begin{array}{ll}
\left.\frac{\partial h}{\partial x}\right|_{x=0} y(0, t)+\left.h(0, t) \frac{\partial y}{\partial x}\right|_{x=0} & =g_{0}(t) \\
\left.\frac{\partial h}{\partial x}\right|_{x=l} y(l, t)+\left.h(l, t) \frac{\partial y}{\partial x}\right|_{x=l} & =g_{l}(t)
\end{array}\right.\right.
$$

- Robin boundary condition: the same procedure as shown above.

Taking a closer look at the transformation of boundary conditions, one immediately see that the von Neumann boundary condition transforms to a Robin boundary condition with time-dependent coefficients. Unfortunately, treating Robin boundary value problems are much more difficult than von Neumann problems, even if the boundary is fixed[77]. However, remember that, we convert a von Neumann problem to a Dirichlet problem by differentiating both sides of the evolution equation supplemented by von Neumann boundary conditions. Rather than working with $Y(x, t)$ which is subjected to a boundary condition of second type, we can investigate the dynamics of $\partial_{x} Y$, which is subjected to the Dirichlet boundary condition. The big gain is that the Dirichlet boundary condition remains Dirichlet after this transformation.

In the next sections of this chapter we propose solutions to equations subjected to this kind of boundary condition, for $\alpha=0.5$ and $\alpha=1$. Because such a solution settles the bond-dynamics of a free chain, which is of importance in our study (see Chapters 5 and 
6 ), we represent the dynamical variable by $\vec{b}$. In particular, we want to find solutions of the equation:

$$
\partial_{\tau} \vec{b}=\partial_{\sigma}^{2} \vec{b}+(\dot{N} N) \sigma \partial_{\sigma} \vec{b}+\vec{\Psi}(\sigma, \tau)
$$

with the homogeneous boundary condition:

$$
\begin{gathered}
\vec{b}(0, \tau)=0 \\
\vec{b}(1, \tau)=0 .
\end{gathered}
$$




\section{Bibliography}

[1] George G. Odian. Principles of polymerization.

[2] Paul J. Flory. Principles of polymer chemistry. Cornell University Press, 1953.

[3] Thomas D Pollard and Gary G Borisy. Cellular Motility Driven by Assembly and Disassembly of Actin Filaments. Cell, 112(4):453-465, 2003.

[4] Thomas D. Pollard. Rate constants for the reactions of ATP-and ADP-actin with the ends of actin filaments. Journal of Cell Biology, 103(6):2747, 1986.

[5] J A Cooper. The Role of Actin Polymerization in Cell Motility. Annual Review of Physiology, 53(1):585-605, 1991.

[6] J B Marchand, P Moreau, A Paoletti, P Cossart, M F Carlier, and D Pantaloni. Actin-based movement of Listeria monocytogenes: actin assembly results from the local maintenance of uncapped filament barbed ends at the bacterium surface. The Journal of Cell Biology, 130(2), 1995.

[7] D A Schafer, P B Jennings, and J A Cooper. Dynamics of capping protein and actin assembly in vitro: uncapping barbed ends by polyphosphoinositides. The Journal of cell biology, 135(1):169-79, 1996.

[8] Philip A. Kuhlman. Dynamic changes in the length distribution of actin filaments during polymerization can be modulated by barbed end capping proteins. Cell Motility and the Cytoskeleton, 61(1):1-8, 2005.

[9] Michael SZWARC. Living' Polymers. Nature, 178(4543):1168-1169, 1956.

[10] Henry L. Hsieh and Roderic P. Quirk. Anionic polymerization : principles and practical applications. Marcel Dekker, 1996.

[11] Nikos Hadjichristidis, Akira Hirao, Yasuyuki Tezuka, and Filip Du Prez, editors. Complex Macromolecular Architectures. John Wiley \& Sons (Asia) Pte Ltd, Singapore, 2011. 
[12] Prince E. Rouse. A Theory of the Linear Viscoelastic Properties of Dilute Solutions of Coiling Polymers. The Journal of Chemical Physics, 21(7):1272-1280, 1953. ISSN 0021-9606.

[13] Bruno H. Zimm. Dynamics of Polymer Molecules in Dilute Solution: Viscoelasticity, Flow Birefringence and Dielectric Loss. The Journal of Chemical Physics, 24(2): 269-278, 1956.

[14] P. G. de Gennes and P. G. Reptation of a Polymer Chain in the Presence of Fixed Obstacles. The Journal of Chemical Physics, 55(2):572-579, 1971.

[15] Masao Doi and S. F. Edwards. The Theory of Polymer Dynamcis. Clarendon Press, 1988.

[16] Daniel T. Gillespie. Chemical Langevin equation. Journal of Chemical Physics, 113 (1):297-306, 2000.

[17] D. C. TIMM and J. W. RACHOW. Description of Polymerization Dynamics by Using Population Density. In Chemical Reaction Engineering-II, pages 122-136. ACS Publications, 1974.

[18] H. Scott Fogler. Elements of chemical reaction engineering. Prentice Hall, 2016.

[19] Michael F. Froix, D. J. Williams, and Andreas O. Goedde. NMR Relaxation Time Studies of Polystyrene. Macromolecules, 9(2):354-358, 1976.

[20] Julie Plastino, Ioannis Lelidis, Jacques Prost, and Ccile Sykes. The effect of diffusion, depolymerization and nucleation promoting factors on actin gel growth. European Biophysics Journal, 33(4):310-320, 2004. ISSN 0175-7571.

[21] F Gittes, B Mickey, J Nettleton, and J Howard. Flexural rigidity of microtubules and actin filaments measured from thermal fluctuations in shape. The Journal of Cell Biology, 120(4), 1993.

[22] David E. Clapham. Calcium Signaling. Cell, 131(6):1047-1058, 2007.

[23] Boris N. Kholodenko. Cell-signalling dynamics in time and space. Nature Reviews Molecular Cell Biology, 7(3):165-176, 2006.

[24] Hanna Engelke, Doris Heinrich, and Joachim O. Rädler. Probing GFP-actin diffusion in living cells using fluorescence correlation spectroscopy. Physical Biology, 7 (4):046014, 2010.

[25] Yi Fan, Sandeepa M. Eswarappa, Masahiro Hitomi, and Paul L. Fox. Myo1c facilitates G-actin transport to the leading edge of migrating endothelial cells. The Journal of Cell Biology, 198(1), 2012. 
[26] Johan T Padding. THEORY OF POLYMER DYNAMICS. Han-sur-Lesse winterschool, 2005.

[27] Don S. Lemons and Anthony Gythiel. Paul Langevin's 1908 paper On the Theory of Brownian Motion [Sur la théorie du mouvement brownien, C. R. Acad. Sci. (Paris) 146, 530533 (1908)]. http://jobs.aapt.org/, 1998.

[28] Daniel R. Gossett, Westbrook M. Weaver, Albert J. Mach, Soojung Claire Hur, Henry Tat Kwong Tse, Wonhee Lee, Hamed Amini, and Dino Di Carlo. Label-free cell separation and sorting in microfluidic systems. Analytical and Bioanalytical Chemistry, 397(8):3249-3267, 2010.

[29] H.A. Stone, A.D. Stroock and A. Ajdari. Engineering Flows in Small Devices: Microfluidics Toward a Lab-on-a-Chip. Annu. Rev. Fluid Mech, 36(1):347-382, 2004.

[30] Pierre giles De Gennes. Scaling Concepts in Polymer Physics. Cornell University Press, first edition, 1979.

[31] R. Shusterman, S. Alon, T. Gavrinyov, and O. Krichevsky. Monomer dynamics in double- and single-stranded DNA polymers. Physical Review Letters, 92(January): 048303, 2004.

[32] K. L. Sebastian. Pulling a polymer out of a potential well and the mechanical unzipping of DNA. Physical Review E - Statistical Physics, Plasmas, Fluids, and Related Interdisciplinary Topics, 62(1 B):1128-1132, 2000.

[33] T. Glomann, G. J. Schneider, J. Allgaier, A. Radulescu, W. Lohstroh, B. Farago, and D. Richter. Microscopic dynamics of polyethylene glycol chains interacting with silica nanoparticles. Physical Review Letters, 110(17):1-5, 2013.

[34] John F Marko and Eric D Siggia. Stretching DNA. Macromolecules, 28(26):87598770, 1995.

[35] Takahiro Sakaue, Takuya Saito, and Hirofumi Wada. Dragging a polymer in a viscous fluid: Steady state and transient. Physical Review E, 86(1):011804, 2012.

[36] Lawrence C. Evans. Partial differential equations. American Mathematical Society, 2010.

[37] Dean G. Duffy. Green'S Functions With Applications. 2015.

[38] Francesco Del Giudice, Vincenzo Calcagno, Valeria Esposito Taliento, Francesco Greco, Paolo Antonio Netti, and Pier Luca Maffettone. Relaxation time of polyelectrolyte solutions: When $\mu$-rheometry steps in charge. Journal of Rheology, 61 (1):13-21, 2017. 
[39] T.K. Sinha and J.P. Gupta. Solidification in an annulus. International Journal of Heat and Mass Transfer, 25(11):1771-1773, 1982.

[40] J.R.A. Pearson. On the melting of solids near a hot moving interface, with particular reference to beds of granular polymers. International Journal of Heat and Mass Transfer, 19(4):405-411, 1976.

[41] HG Landau. Heat conduction in a melting solid. Quarterly of Applied Mathematics, 1950.

[42] Adam Kucera. Mathematics of moving boundary problems in diffusion. 1985.

[43] I. Bronstein, Y. Israel, E. Kepten, S. Mai, Y. Shav-Tal, E. Barkai, and Y. Garini. Transient Anomalous Diffusion of Telomeres in the Nucleus of Mammalian Cells. Physical Review Letters, 103(1):018102, 2009.

[44] Julia F. Reverey, Jae-Hyung Jeon, Han Bao, Matthias Leippe, Ralf Metzler, and Christine Selhuber-Unkel. Superdiffusion dominates intracellular particle motion in the supercrowded cytoplasm of pathogenic Acanthamoeba castellanii. Scientific Reports, 5(1):11690, 2015.

[45] Krzysztof Matyjaszewski, Chih-Hwa Lin, Angélique Bon, and Jason S. Xiang. Salt and solvent effects in living carbocationic polymerization. Macromolecular Symposia, 85(1):65-78, 1994.

[46] Richard P. N. Veregin, Peter G. Odell, Lora M. Michalak, and Michael K. Georges. The Pivotal Role of Excess Nitroxide Radical in Living Free Radical Polymerizations with Narrow Polydispersity. Macromolecules, 29(8):2746-2754, 1996.

[47] Daniel T Gillespie. A general method for numerically simulating the stochastic time evolution of coupled chemical reactions. Journal of Computational Physics, 22(4): 403-434, 1976.

[48] H. C. Ottinger. Stochastic Processes In Polymeric Fluids, volume 1. 1996.

[49] J. Honerkamp. Stochastic dynamical systems : concepts, numerical methods, data analysis. VCH, 1994.

[50] Charles Van Loan. Computational Frameworks for the Fast Fourier Transform. Society for Industrial and Applied Mathematics, 1992.

[51] M. Frigo and S.G. Johnson. The Design and Implementation of FFTW3. Proceedings of the IEEE, 93(2):216-231, 2005.

[52] Stanley J. Farlow. Partial Differential Equations for Scientists and Engineers. Dover Publication, INC, New York, 1993. 
[53] J. B. Walsh. Finite element methods for parabolic stochastic PDE's. Potential Analysis, 23(1):1-43, 2005.

[54] A. Jentzen and P. E. Kloeden. The Numerical Approximation of Stochastic Partial Differential Equations. Milan Journal of Mathematics, 77(1):205-244, 2009.

[55] Arnulf Jentzen and Peter E. Kloeden. Taylor Approximations for Stochastic Partial Differential Equations. Society for Industrial and Applied Mathematics, 2011.

[56] Andrea Barth. Stochastic Partial Differential Equations : Approximations and Applications. dissertation, University of Oslo, 2009. URL https://www.duo.uio.no/ bitstream/handle/10852/10669/Barth_publ . pdf? sequence=3\&isAllowed=y.

[57] Carl M. Bender and Steven A. Orszag. Advanced Mathematical Methods for Scientists and Engineers I : Asymptotic Methods and Perturbation Theory. Springer New York, 1999.

[58] C. Gros. Complex and Adaptive Dynamical Systems: A Primer. 2008.

[59] KL Ngai, S Mashimo, and G Fytas. Intercomparisons of dielectric relaxation, dynamic light scattering, and viscoelastic properties of the local segmental motion in amorphous polymers. Macromolecules, 1988.

[60] Pierre-Gilles de Gennes. Relaxation Anomalies in Linear Polymer Melts. Macromolecules, 35(9):3785-3786, 2002.

[61] Emmanuel Paspalakis, Va a Harmandaris, Vg G Mavrantzas, High Temperature, and Chemical Processes. Segmental dynamics in polyethylene melts through atomistic molecular dynamics simulations. Recent Research Topics and Developments in Chemical Physics: From Quantum Scale to Macroscale, 661(2):179-196, 2008.

[62] Claude Cohen-Tannoudji, Bernard Diu, and Franck Laloe. Quantum mechanics. Wiley, 1977.

[63] Andrei D. Polyanin and Vladimir E. Nazaikinskii. Handbook of linear partial differential equations for engineers and scientists.

[64] Milton Abramowitz and Irene A. Stegun. Handbook of Mathematical Functions: with Formulas, Graphs, and Mathematical Tables. Dover Publication, 1965.

[65] Zeev Schuss. Theory and applications of stochastic processes : an analytical approach. Springer, 2010.

[66] M D Wang, H Yin, R Landick, J Gelles, and S M Block. Stretching DNA with optical tweezers. Biophysical journal, 72(3):1335-46, 1997. 
[67] Charles S Epstein and Ariana J Mann. Measurement of the DNA Spring Constant Using Optical Tweezers. 2012.

[68] Oliver Otto, Sebastian Sturm, Nadanai Laohakunakorn, Ulrich F Keyser, and Klaus Kroy. Rapid internal contraction boosts DNA friction. Nature communications, 4 : 1780, 2013.

[69] Roman D. Bulushev, Sanjin Marion, and Aleksandra Radenovic. Relevance of the Drag Force during Controlled Translocation of a DNAProtein Complex through a Glass Nanocapillary. Nano Letters, 15(10):7118-7125, 2015.

[70] Takahiro Sakaue. Nonequilibrium dynamics of polymer translocation and straightening. Physical Review E - Statistical, Nonlinear, and Soft Matter Physics, 76(2): $1-7,2007$.

[71] Takahiro Sakaue. Sucking genes into pores: Insight into driven translocation. Physical Review E - Statistical, Nonlinear, and Soft Matter Physics, 81(4):1-6, 2010.

[72] John M. Dealy and Kurt F. Wissbrun. Steady Simple Shear Flow and the Viscometric Functions. In Melt Rheology and Its Role in Plastics Processing, pages 153-178. Springer Netherlands, Dordrecht, 1999.

[73] R. Byron (Robert Byron) Bird. Dynamics of polymeric liquids. Wiley, 1987.

[74] JL White. Dynamics of viscoelastic fluids, melt fracture, and the rheology of fiber spinning. Journal of Applied Polymer Science, 1964.

[75] Oskar Hallatschek, Erwin Frey, and Klaus Kroy. Propagation and relaxation of tension in stiff polymers. Physical Review E - Statistical, Nonlinear, and Soft Matter Physics, 94(7):1-20, 2005.

[76] Oskar Hallatschek, Erwin Frey, and Klaus Kroy. Tension dynamics in semiflexible polymers. II. Scaling solutions and applications. Physical Review E - Statistical, Nonlinear, and Soft Matter Physics, 75(3):1-20, 2007.

[77] K. (Kenneth) Eriksson. Computational differential equations. Cambridge University Press, 1996. 
CV 
Ali Malek

Pfeifferstr. 19

34121 Kassel, Deutschland

(+49) 176-324 95457

Beruflicher

Wissenschaftlicher Mitarbeiter

Nov.2017-Jetzt

Werdegang

Institut für nicht lineare Dynamik, Georg-August-Universität Göttingen

Software Entwickler

Jan.2017-Okt.2017

Tachycon GmbH, Frankfurt am Main

Wissenschaftlicher Mitarbeiter

Okt.2012-Jun.2016

Institut für theoretische Physik, Georg-Augugst-Universität Göttingen

STUDIUM

Promotion,

Okt.2012-Mär.2018

Georg-Augugst-Universität Göttingen

Doktorarbeit: "Conformational Dynamics of Living Polymers"

Betreuer: Prof. Dr. Reiner Kree

Master,

Georg-Augugst-Universität Göttingen,

Okt.2010-Jul.2012

Bachelor,

2006-2010

Sharif Univeristy of Technology, Teheran, Iran,

\section{VERÖFFENTLICHUNGEN}

"Growth-induced dynamical transitions in a living polymer chain" Submitted on 25.02.2018, Physical Review Letters, (Under Review) 